Utah State University

DigitalCommons@USU

$5-2009$

\title{
Terrain-Relative and Beacon-Relative Navigation for Lunar Powered Descent and Landing
}

Daniel Porter Christensen

Utah State University

Follow this and additional works at: https://digitalcommons.usu.edu/etd

Part of the Aerospace Engineering Commons

\section{Recommended Citation}

Christensen, Daniel Porter, "Terrain-Relative and Beacon-Relative Navigation for Lunar Powered Descent and Landing" (2009). All Graduate Theses and Dissertations. 253.

https://digitalcommons.usu.edu/etd/253

This Thesis is brought to you for free and open access by the Graduate Studies at DigitalCommons@USU. It has been accepted for inclusion in All Graduate Theses and Dissertations by an authorized administrator of DigitalCommons@USU. For more information, please contact digitalcommons@usu.edu.

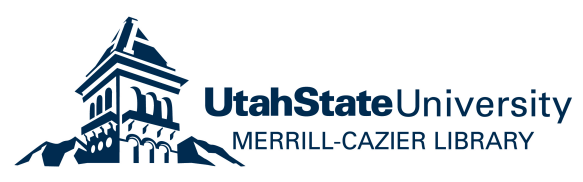


TERRAIN-RELATIVE AND BEACON-RELATIVE NAVIGATION FOR LUNAR POWERED DESCENT AND LANDING

\author{
by \\ Daniel P. Christensen \\ A thesis submitted in partial fulfillment \\ of the requirements for the degree \\ of \\ MASTER OF SCIENCE \\ in \\ Mechanical Engineering
}

Approved:

Dr. David K. Geller

Major Professor

Dr. R. Rees Fullmer

Committee Member

Dr. Barton L. Smith

Dr. Byron R. Burnham

Committee Member

Dean of Graduate Studies

UTAH STATE UNIVERSITY

Logan, Utah 
Copyright (c) Daniel P. Christensen 2009

All Rights Reserved 


\begin{abstract}
Terrain-Relative and Beacon-Relative Navigation for Lunar Powered Descent and Landing by

Daniel P. Christensen, Master of Science

Utah State University, 2009

Major Professor: Dr. David K. Geller

Department: Mechanical and Aerospace Engineering

As NASA prepares to return humans to the moon and establish a long-term presence on the surface, technologies must be developed to access previously unvisited terrain regardless of the condition. Among these technologies is a guidance, navigation and control (GNC) system capable of safely and precisely delivering a spacecraft, whether manned or robotic, to a predetermined landing area. This thesis presents detailed research of both terrain-relative navigation using a terrain-scanning instrument and beacon-relative radiometric navigation using beacons in lunar orbit or on the surface of the moon. The models for these sensors are developed along with a baseline sensor suite that includes an altimeter, IMU, velocimeter, and star camera. Linear covariance analysis is used to rapidly perform the trade studies relevant to this problem and to provide the navigation performance data necessary to determine which navigation method is best suited to support a $100 \mathrm{~m} \mathrm{3- \sigma}$ navigation requirement for landing anytime and anywhere on the moon.
\end{abstract}

(90 pages) 


\section{Acknowledgments}

First of all, I would like to thank the faculty at Utah State University for their time, talent, and expertise during my years at the university. Most of all, I would like to thank my advisor, Dr. David Geller. I feel that I have been fortunate to have associated so closely with him for the past three years. He truly is a natural teacher and it would seem that there is not an end to the subjects I could learn from him. I doubt that I ever will be able to repay him for all the time and effort he has invested in my education and in the projects we have worked on together, so it would seem that the only recourse is to find ways to pass it on.

Also, I need to thank NASA and the ALHAT GNC community for their funding and the support they gave in developing the models and definitions used in this research and for sharing their insight into its results.

Lastly, I would like to thank my family for their support throughout this entire endeavor. First, I am grateful that my parents, Ron and Elise Christensen, instilled in me an understanding of the many benefits of a good education and then encouraged me through all the difficult times that came along with it. Most of all, I would like to thank my wife, Lacey Christensen, whom I met and married along the way. She has been an incredible support and motivating force throughout all the difficult days we have had. I am quite certain that the past and current circumstances would not have worked out as well as they have without her support and voice of reason.

Daniel P. Christensen 


\section{Contents}

Abstract $\ldots \ldots \ldots \ldots \ldots \ldots \ldots \ldots \ldots \ldots \ldots \ldots \ldots \ldots \ldots \ldots \ldots \ldots \ldots$

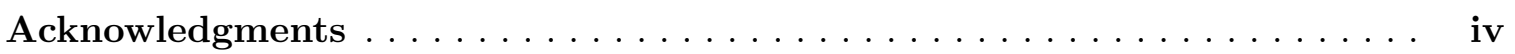

List of Tables $\ldots \ldots \ldots \ldots \ldots \ldots \ldots \ldots \ldots \ldots \ldots \ldots \ldots \ldots \ldots \ldots \ldots$

List of Figures $\ldots \ldots \ldots \ldots \ldots \ldots \ldots \ldots \ldots \ldots \ldots \ldots \ldots \ldots \ldots \ldots$

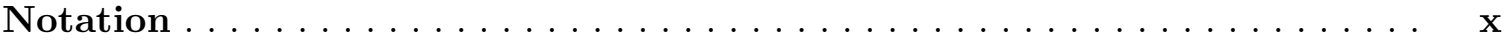

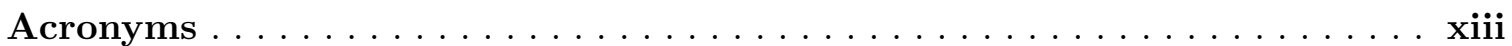

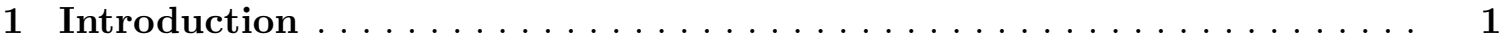

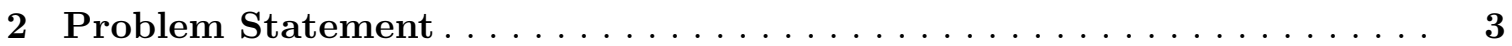

3 Literature Survey . . . . . . . . . . . . . . . . . . 4

3.1 Lunar Navigation Heritage . . . . . . . . . . . . . . . . . . . . 4

3.2 The ALHAT Project Assumptions . . . . . . . . . . . . . . . 4

3.2 .1 Trajectories ...................... 5

3.2 .2 Initial Errors . . . . . . . . . . . . . . . . . 8

3.2 .3 The Lunar Environment . . . . . . . . . . . . . . . . 9

3.2 .4 Baseline Sensors . . . . . . . . . . . . . . . . . . 9

3.2 .5 Reference Frames . . . . . . . . . . . . . . . . . . . . . . . . . 11

3.3 Orbital Elements and Covariances _. . . . . . . . . . . . . . . 12

3.4 Frozen Lunar Orbits . . . . . . . . . . . . . . . . . . . . . . . . 13

3.5 Terrain-Relative Navigation . . . . . . . . . . . . . . . . . . . . . 13

3.6 Radiometric Navigation . . . . . . . . . . . . . . . . . . . . 15

3.7 Kalman Filtering . . . . . . . . . . . . . . . . . . . . 17

3.8 Covariance Analysis . . . . . . . . . . . . . . . . . . . . . 18

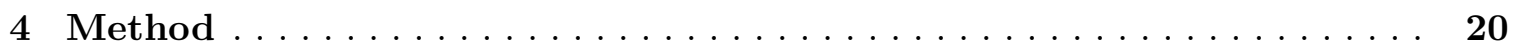

4.1 Linear Covariance Simulation . . . . . . . . . . . . . . . . . . . . . 20

4.2 Dynamics and Environment Truth Models . . . . . . . . . . . . . . . 23

4.3 Sensor Truth Models . . . . . . . . . . . . . . . . . . . . . 24

4.4 Navigation Algorithm . . . . . . . . . . . . . . . . . . . . 29

4.5 Metrics . . . . . . . . . . . . . . . . . . . 32

4.6 Requirements . . . . . . . . . . . . . . . . . 32 


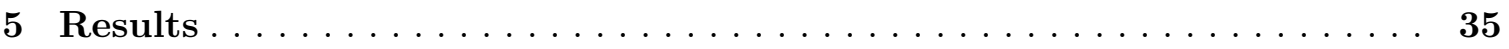

5.1 Terrain-Relative Navigation Studies _ . . . . . . . . . . . . . 35

5.1 .1 TRN Operating Range Study . . . . . . . . . . . . . . . . 35

5.1 .2 TRN Trajectory Study . . . . . . . . . . . . . . . . 38

5.1 .3 Map Resolution . . . . . . . . . . . . . . . . . . . . . . . . . 39

5.1 .4 Measurement Rate and Outages . . . . . . . . . . . . . . 41

5.2 Radiometric Navigation Studies . . . . . . . . . . . . . . . . . . . . . . . . 42

5.2.1 Single Orbiting Beacon Study . . . . . . . . . . . . . . . . . . 43

5.2.2 Two Coplanar Orbiting Beacons Study . . . . . . . . . . . . . . 47

5.2 .3 Single Surface Beacon Study _. . . . . . . . . . . . . . . . . 49

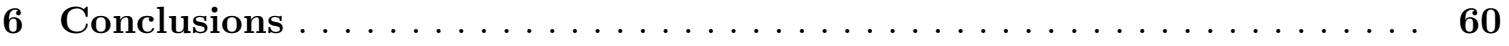

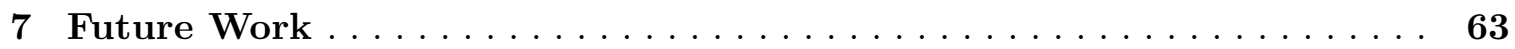

7.1 Additional Terrain-Relative Navigation Work . . . . . . . . . . . . . . 63

7.2 Additional Radiometric Navigation Work . . . . . . . . . . . . . . 63

7.3 Guidance Implementation . . . . . . . . . . . . . . . . . . . . . 64

7.4 Modeling Improvement . . . . . . . . . . . . . . . . . . . . . . 64

7.5 Reduced-State Filter . . . . . . . . . . . . . . . . . . . . . . 64

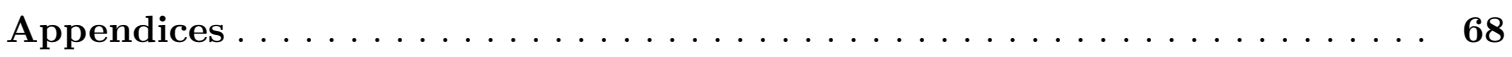

Appendix A Matrix Partial Derivatives . . . . . . . . . . . . 69

A.1 Covariance Propagation Matrices . . . . . . . . . . . . . . 69

A.2 Measurement Sensitivity Matrices . . . . . . . . . . . . 70

Appendix B Driving Factors for Radiometric Navigation Performance . . . . . 73

B.1 Single Orbiting Beacon Case Study . . . . . . . . . . . . . . . . . 73

B.2 Single Surface Beacon Case Study . . . . . . . . . . . . 76 


\section{List of Tables}

Table $\quad$ Page

3.1 Reduced Trajectory Trade Space . . . . . . . . . . . . . . . . 7

3.2 Initial Lander Covariance $\ldots \ldots \ldots \ldots$

3.3 Baseline Sensor Suite Parameters . . . . . . . . . . . . . . . . . . 10

3.4 Baseline TRN Sensor Parameters . . . . . . . . . . . . . . . . . . 15

3.5 Baseline Radiometric Parameters . . . . . . . . . . . . . . . . . . . . 16

4.1 List of States in the Linear Covariance Simulation _ . . . . . . . . . . . 21

4.2 Navigation Requirements . . . . . . . . . . . . . . . . 33

5.1 LRS Orbital Elements and Initial Variance . . . . . . . . . . . . . . . 42

5.2 Statistics of the navigation performance for the single orbiting beacon case $\quad 45$

5.3 Statistics of the Navigation Performance for the Two Orbiting Beacon Case 48 


\section{List of Figures}

Figure $\quad$ Page

3.1 Phases of the final descent. . . . . . . . . . . . . . 5

3.2 General trajectory geometry. . . . . . . . . . . . . 6

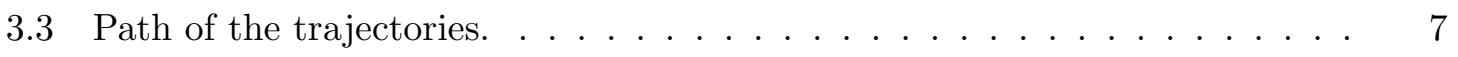

3.4 Monte Carlo block diagram. . . . . . . . . . . . . . . . . 19

4.1 Effect of TRN misalignment. . . . . . . . . . . . . . 27

4.2 Elevation angle constraints for radiometric signals. . . . . . . . . . . 29

4.3 Baseline navigation. . . . . . . . . . . . . . . 34

5.1 Possible TRN operating ranges. . . . . . . . . . . . . . 37

5.2 Effect of varying the operating range of the TRN sensor. . . . . . . . . . 37

5.3 Effect of varying the trajectory type on TRN performance. . . . . . . . 38

5.4 Effect of varying the map resolution. . . . . . . . . . . . . 39

5.5 Navigation results for outpost mission. . . . . . . . . . . . . . 40

5.6 Effect of varying the TRN measurement rate on TRN performance. . . . . . 41

5.7 Grids for LCT placement. . . . . . . . . . . . . . . . . 43

5.8 Time history of the navigation error using measurements to one LRS. . . . 44

5.9 Navigation results at PDI using radiometric measurements to an LRS. . . . 45

5.10 Sensitivity of navigation relative to one LRS. . . . . . . . . . . 47

5.11 Navigation results at PDI using measurements to two LRSs. . . . . . . . . 48

5.12 Sensitivity of navigation relative to two LRSs. . . . . . . . . . . . . 50

5.13 Navigation error relative to a coarse grid LCT. . . . . . . . . . . . 51

5.14 Navigation error relative to a fine grid LCT. . . . . . . . . . . 53 
5.15 Sensitivity of coarse grid LCT. . . . . . . . . . . . . . . 54

5.16 Effect of lowering visibility constraint on navigation relative to a coarse grid LCT. . . . . . . . . . . . . . . . . . 55

5.17 Effect of raising visibility constraint on navigation relative to a coarse grid

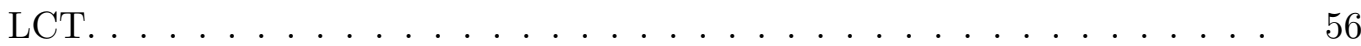

5.18 Sensitivity to trajectory type of navigation relative to a coarse grid LCT. $\quad . \quad 57$

5.19 Sensitivity to trajectory type of navigation relative to a fine grid LCT at throttle-down. . . . . . . . . . . . . . . . 58

5.20 Sensitivity to trajectory type of navigation relative to a fine grid LCT at landing. . . . . . . . . . . . . . . . . . . . 59

B.1 Navigation results from LRS measurements only during final descent. . . . 73

B.2 Duration of tracking between the lander and a single LRS. . . . . . . . . . 74

B.3 Maximum elevation reached by the LRS relative to the lander. . . . . . . . 74

B.4 Navigation results at landing using measurements to a coarse grid LCT. . . 76

B.5 Duration of tracking between the lander and a coarse grid LCT. . . . . . . 77

B.6 Duration of time between the last radiometric measurement to a coarse grid LCT and landing. . . . . . . . . . . . . . . . . 77

B.7 Maximum elevation reached by the lander relative to the LCT. . . . . . . 77 


\section{Notation}

\section{General Symbols}

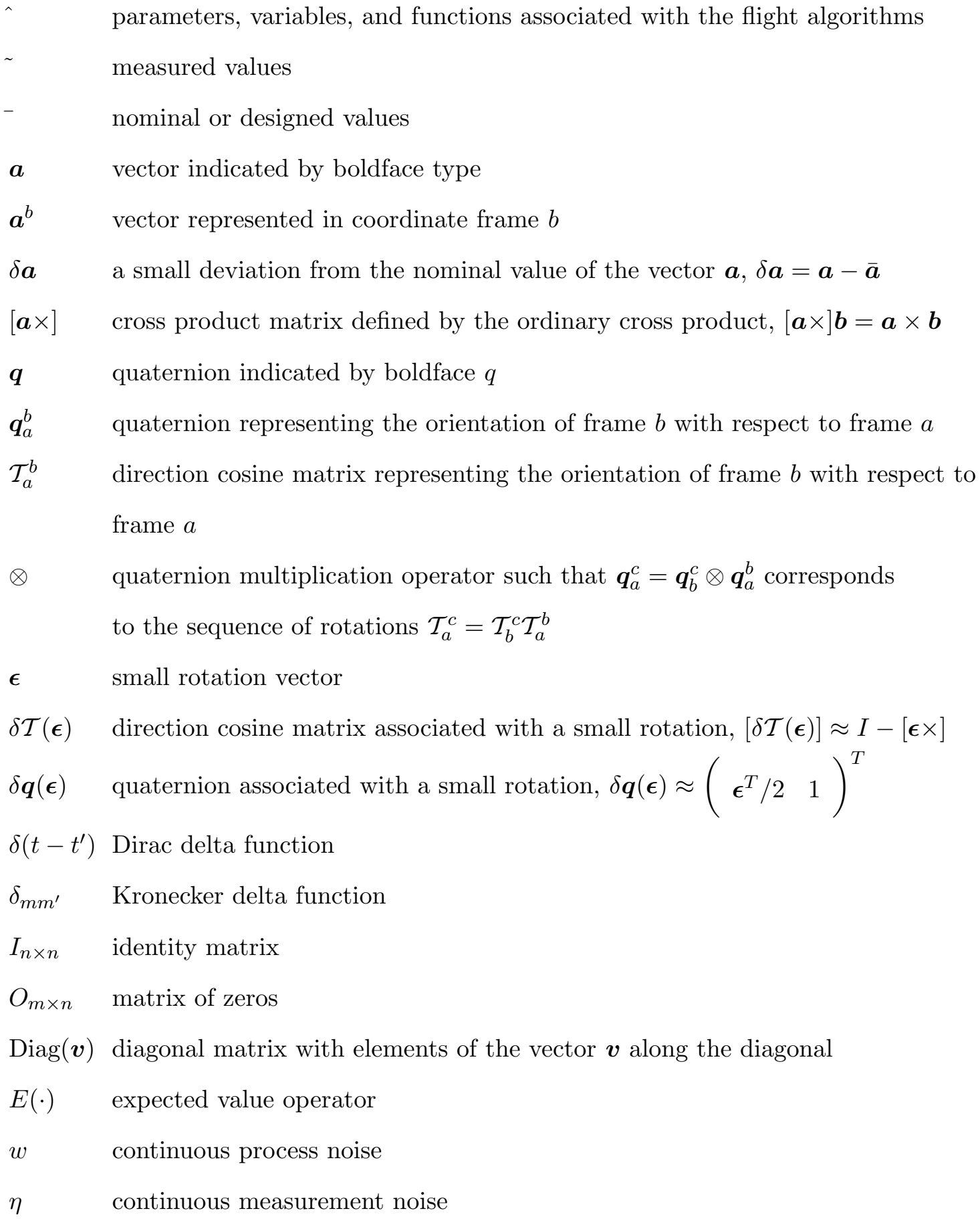




$\begin{array}{ll}\nu & \text { discrete measurement noise } \\ S & \text { covariance of a continuous noise process } \\ R & \text { covariance of a discrete noise process } \\ \tau & \text { time constant } \\ d_{k} & \text { correlation distance } \\ \sigma & \text { standard deviation }\end{array}$

\section{Subscripts}

$\begin{array}{ll}\ell & \text { lander } \\ o & \text { LRS orbiters } \\ r & \text { reference site } \\ s & \text { sensors } \\ \text { accel } & \text { accelerometers } \\ \text { gyro } & \text { gyroscopes } \\ \text { star } & \text { star camera } \\ \text { alt } & \text { altimeter } \\ \text { vel } & \text { velocimeter } \\ \text { trn } & \text { TRN sensor } \\ \text { radio } & \text { radiometric navigation link } \\ \text { grav } & \text { gravitational } \\ \text { map } & \text { onboard map } \\ \text { elv } & \text { terrain elevation }\end{array}$

\section{Superscripts}

$\begin{array}{ll}T & \text { matrix/vector transpose } \\ i & \text { inertial reference frame } \\ \ell & \text { lander body reference frame } \\ s & \text { surface reference frame }\end{array}$




$$
\begin{array}{ll}
\text { star } & \text { star camera reference frame } \\
\text { vel } & \text { velocimeter reference frame } \\
- & \text { before a state update } \\
+ & \text { after a state update }
\end{array}
$$

\section{States}

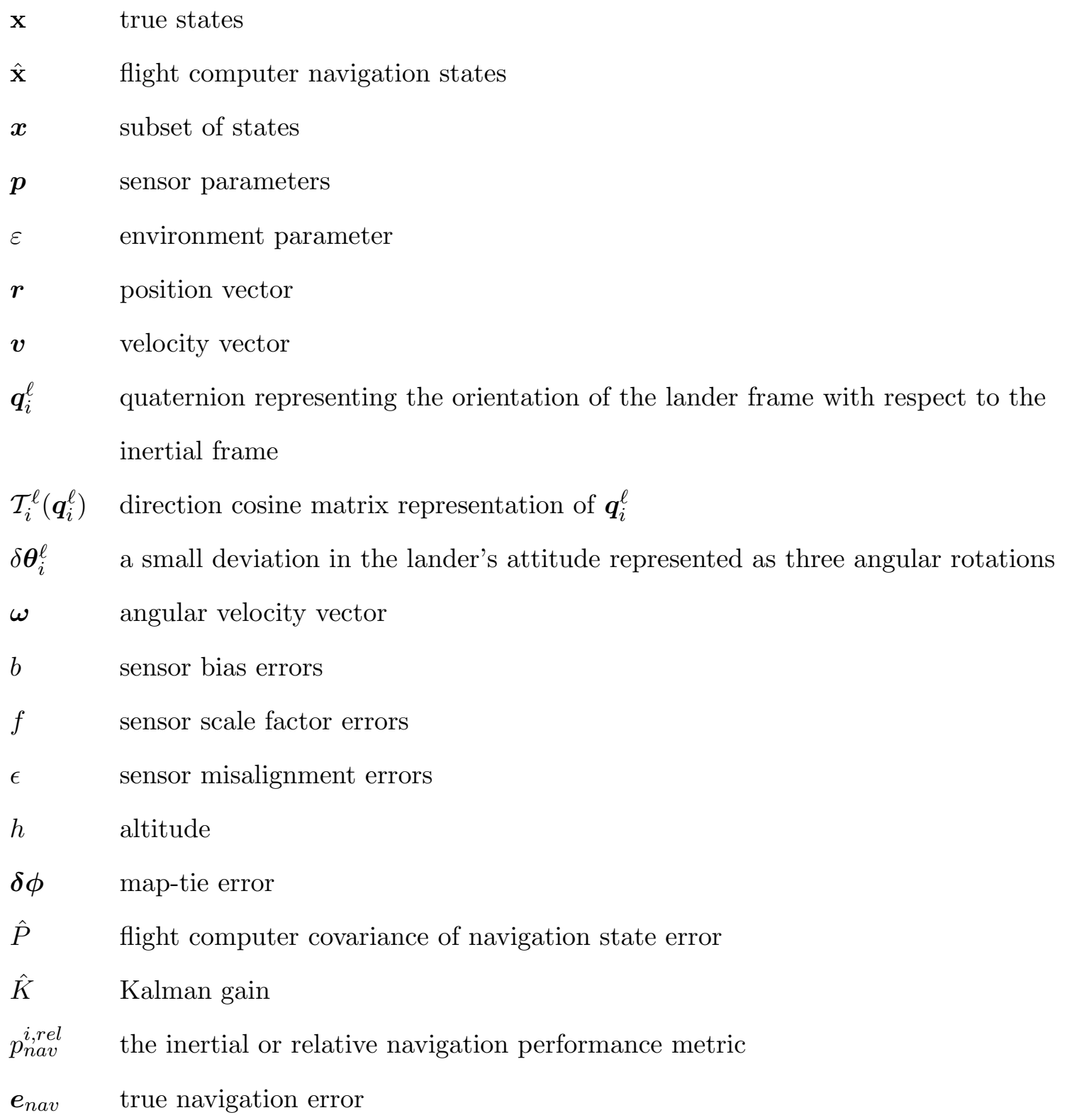




\section{Acronyms}

\begin{tabular}{|c|c|}
\hline ALHAT & Autonomous Landing and Hazard Avoidance Technology \\
\hline $\mathrm{CG}$ & Center of Gravity \\
\hline CCD & Charge Coupled Device \\
\hline DAC1 & First Design Analysis Cycle \\
\hline $\mathrm{EKF}$ & Extended Kalman Filter \\
\hline GNC & Guidance, Navigation, and Control \\
\hline HDA & Hazard Detection and Avoidance \\
\hline IMU & Inertial Measurement Unit \\
\hline JPL & Jet Propulsion Laboratory \\
\hline LCI & Lunar-Centered Inertial \\
\hline $\mathrm{LCT}$ & Lunar Communications Terminal \\
\hline $\mathrm{LN}$ & Lunar Network \\
\hline LRO & Lunar Reconnaissance Orbiter \\
\hline LRS & Lunar Relay Satellite \\
\hline LSF & Lunar Surface Fixed \\
\hline LVLH & Local-Vertical, Local-Horizontal \\
\hline MA & Mean Anomaly \\
\hline NASA & National Aeronautics and Space Administration \\
\hline PDI & Powered Descent Initiation \\
\hline RAAN & Right Ascension of the Ascending Node \\
\hline TRN & Terrain-Relative Navigation \\
\hline
\end{tabular}




\section{Chapter 1 Introduction}

As NASA prepares to return humans to the moon and establish a long-term presence on the surface, technologies must be developed to access previously unvisited terrain regardless of the condition. Among these technologies is a guidance, navigation and control (GNC) system capable of safely and precisely delivering a spacecraft, whether manned or robotic, to a pre-determined landing area with an accuracy of $100 \mathrm{~m}$ or less. Particular areas of interest on the moon include its poles - particularly the South Pole - where data from the Clementine mission indicates that frozen volatiles such as water may have gathered in permanently shadowed craters.

The current state of the art for such a system was established by the Apollo program in the 1960's, which had many operational constraints. These included the limitation of landings to the near-side of the moon, prescribed lighting conditions, and significant intervention from the ground control, and even then landing accuracies were above a kilometer. Soon after it adopted the Vision for Space Exploration, in 2006 NASA initiated a new project termed "Autonomous precision Landing and Hazard detection and Avoidance Technology" (Commonly referred to as ALHAT) to develop a new state of the art GNC system and its supporting hardware and software. The highest priorities of the ALHAT project were to develop the technology needed to autonomously detect hazards in the landing area, select an optimal landing site, and then navigate to the selected landing site. The project aims to accomplish this while minimizing or eliminating all operational constraints.

During the beginning stages of the program it was necessary to make several important decisions such as what role the crew would play in the landing, what type of landing trajectory should be used, and what the landing requirements were to be. The role the crew would play was temporarily eliminated for the first design cycle because the ALHAT 
mission statement included robotic and cargo class missions, which would likely be the first to fly. This was done with the understanding that the system would be modified for crewed missions to provide the astronauts with the best information possible during the descent. It was decided that trajectory selection would require extensive consideration of optimal fuel usage, navigation accuracy, and crew preferences. The requirements for landing were decided to be "within 10's of meters anytime, anywhere if there are lunar navigation assets in place and/or precise lunar maps" [1].

Another important decision that the team needed to make was which sensors were best suited for achieving the goals of ALHAT. Early on, a baseline sensor suite was adopted which included an inertial measurement unit (IMU) with three gyroscopes and three accelerometers, as well as a star-camera for attitude determination, a velocimeter to measure the terrain-relative velocity of the lander, and an altimeter. Though these sensors were all very helpful for a precision landing, a method to further decrease the navigation errors was necessary to meet the requirements for a precision landing. Two possible solutions were radiometric measurements to lunar navigation assets such as surface and orbiting beacons, or terrain-relative navigation (TRN) using a method to correlate visible terrain with onboard maps. Because of the likelihood that there would be missions in which surface or orbiting assets would be non-existent or they would be unavailable at critical times due to their positions, it was decided that analysis should proceed with the assumption that the precision landing requirements should be attainable without the aid of radiometric navigation. Thus the baseline for the ALHAT project now includes TRN to aid in the achievement of the precision landing requirements. Despite this, however, the benefit of the radiometric measurements when navigation assets are available remains of great interest. 


\section{Chapter 2}

\section{Problem Statement}

The thesis of this research is that the ALHAT project is correct in assuming that terrain-relative navigation is more flexible and reliable for lunar landings than surface and orbiting beacon-relative navigation. To test this thesis, the capability of these two navigation methods to support estimation of the position and velocity of the lander relative to the landing site is evaluated using covariance analysis techniques. Both beacon- and terrainrelative navigation methods are used in conjunction with the baseline ALHAT sensor suite and an extended Kalman filter. Each phase included sensitivity studies of the navigation accuracy to multiple parameters.

The primary goals of the first phase of the research are to provide insight into the most beneficial time to use the TRN sensor and then estimate the navigation accuracy that can be achieved with TRN. Secondary goals of this phase are to provide estimates of the sensitivity of the navigation results to the type of descent trajectory, the accuracy of the TRN sensor, the measurement rate, and the initial navigation errors.

The primary goals in the second phase are to evaluate the navigation performance during powered descent when radiometric measurements are taken to orbiting beacons called Lunar Relay Satellites (LRSs) or surface beacons surveyed into the lunar map called Lunar Communication Terminals (LCTs). The secondary goals of this phase are to provide es-

timates of the sensitivity of radiometric navigation results to several parameters including the accuracy of the radiometric measurements, the initial navigation errors, the locations of the lunar navigation assets, and the type of the descent trajectory.

Finally, a comparison is drawn between the navigation results to determine which method is most accurate. These accuracies are then weighed against the results of the sensitivity studies to determine which method is more flexible and reliable. 


\section{Chapter 3 \\ Literature Survey}

\subsection{Lunar Navigation Heritage}

The Heritage for the ALHAT program began in the 1950's and 1960's when NASA was studying the feasibility of a manned lunar mission. The widely-publicized Apollo missions set the precedence for accomplishing the feat. Room remains, however, for improvement with the advancement in computer and GNC technology. The navigation methods used onboard the Apollo consisted purely of inertial navigation using a gyroscopically-stabilized platform with accelerometers along with star measurements to periodically correct the inertial platform [2]. More sophisticated navigation techniques would be required to visit more difficult terrain.

In 1989 President George H. W. Bush announced the Space Exploration Initiative, which included goals of the Freedom Space Station and lunar and martian exploration. Though the initiative was not completely successful, some important studies emerged including one into the use of a radiometric navigation architecture similar to the one used in this research. In this study [3], a network consisting of 6 orbiting beacons and a single surface beacon was evaluated by its ability to maintain the ephemeris knowledge of each orbiting beacon and to provide inertial navigation support to the lander as it arrived at the moon, orbited with an altitude of $100 \mathrm{~km}$, and landed. It is difficult, however, to extrapolate these results to different network configurations and landing scenarios.

\subsection{The ALHAT Project Assumptions}

An overview of the choices made in the early stages of the ALHAT project was written by the project manager, Dr. Chirold Epp [1], and was used as the main source for the introduction of this thesis. For the first design analysis cycle (DAC1), multiple parameters 


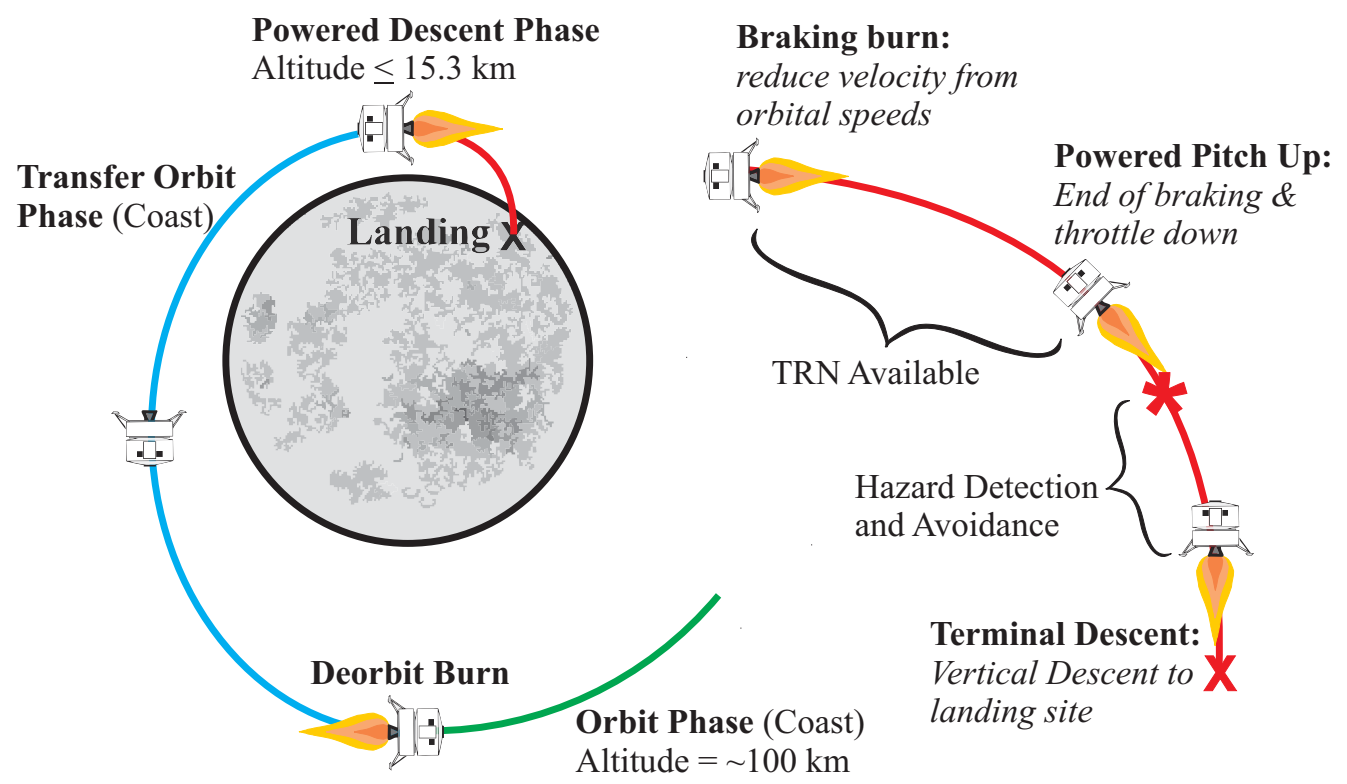

Fig. 3.1: Phases of the final descent [1].

were set as baselines to serve as a reference until further analysis provided the optimal values. These baseline parameters will be discussed in the following subsections.

\subsubsection{Trajectories}

The phases of landing were decided to first include a loiter phase where the lander orbits the moon in a circular orbit at an altitude of $100 \mathrm{~km}$ for a number of orbits after arriving at the moon. Nearly half a revolution prior to landing, a deorbit burn is executed to bring the perilune of the lander to approximately 15.3 kilometers. When the lander approaches perilune, powered descent initiation (PDI) occurs and the thrusters fire until the lander reaches the surface. Toward the end of the powered descent phase, the thrusters throttle down and the vehicle pitches up in the powered pitch-up phase. At approximately this time the Hazard Detection and Avoidance (HDA) phase is initiated. Finally, when the lander is directly over the landing site, it descends vertically for a soft landing. These phases of the descent trajectory are shown in Fig. 3.1.

The complete trajectory trade space consists of 252 trajectories with differing combinations of acceleration levels, slant ranges, and trajectory angles [4]. These parameters are 


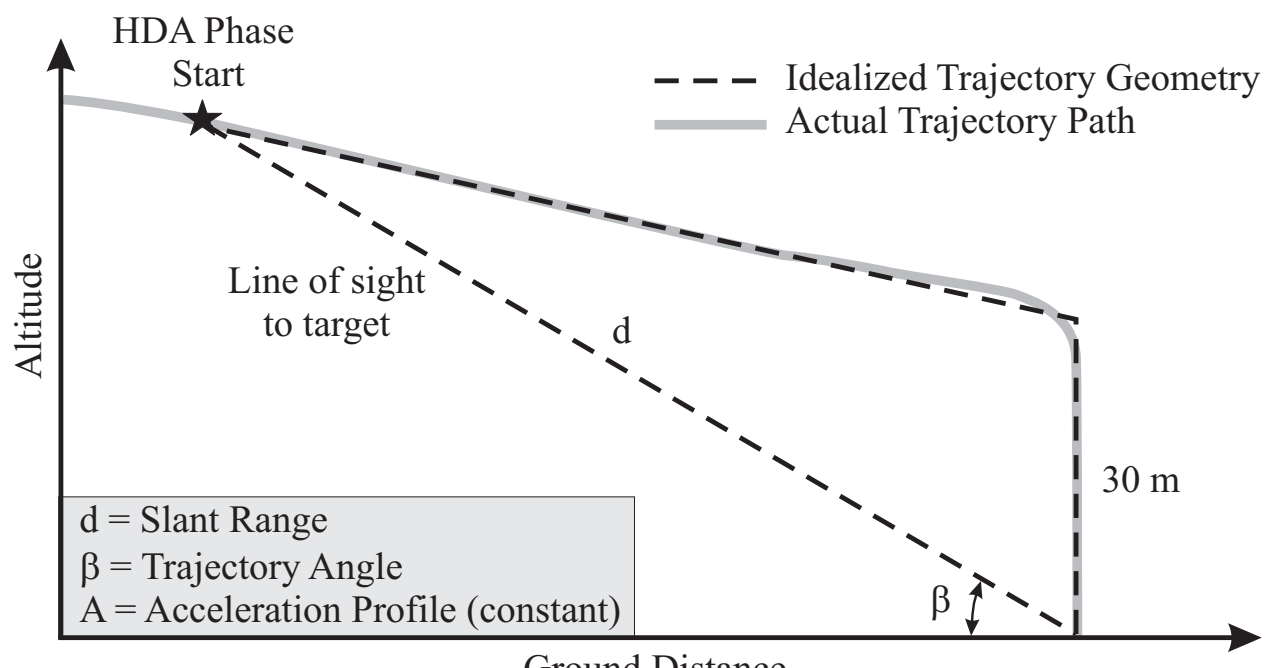

Fig. 3.2: General trajectory geometry.

defined in Fig. 3.2. To reduce the number of runs required to characterize the effect of changing the geometry of the trajectory, a reduced trade space was defined by the ALHAT team with eight trajectories that span the complete trade space. The geometries for each of these trajectories and their nicknames are displayed in Table 3.1 along with their number in the original trajectory trade space. The path followed by each trajectory is plotted in Fig. 3.3. The "Baseline" trajectory was chosen as a reference because it is located in the center of the trade space. The "Apollo-like" trajectory is similar to the original Apollo trajectories because it has a very shallow approach to the landing site. The "Sensor" trajectory descends vertically upon the landing site from an altitude of $1000 \mathrm{~km}$, giving an ample amount of time for the HDA sensor to scan the terrain. The "Cheapo" trajectory minimizes the fuel used in the descent. The "Brakes" trajectory approaches the landing site as efficiently as possible and then descends slowly. The "Slanty" trajectory likely represents the lowest slant angle that would support HDA while the "Peeky" trajectory likely has the highest slant angle that will allow the astronauts onboard the lander to visually scan the terrain through the lander's window. Finally, the "Stretch" trajectory can be compared with the "Baseline" trajectory to isolate the effect of the slant range. 
Table 3.1: Reduced Trajectory Trade Space

\begin{tabular}{lcccr}
\hline \hline Trajectory & $\#$ & A (lunar g's) & $\boldsymbol{\beta}(\mathbf{d e g})$ & $\mathbf{d ~ ( \mathbf { m } )}$ \\
\hline Baseline & 87 & 1.2 & 45 & 1000 \\
Apollo-like & 75 & 1.1 & 15 & 2000 \\
Sensor & 126 & 1.3 & 90 & 1000 \\
Cheapo & 201 & 1.5 & 45 & 800 \\
Brakes & 33 & 1.05 & 45 & 500 \\
Slanty & 104 & 1.2 & 30 & 500 \\
Peeky & 88 & 1.2 & 60 & 1000 \\
Stretch & 75 & 1.2 & 45 & 2000 \\
\hline
\end{tabular}

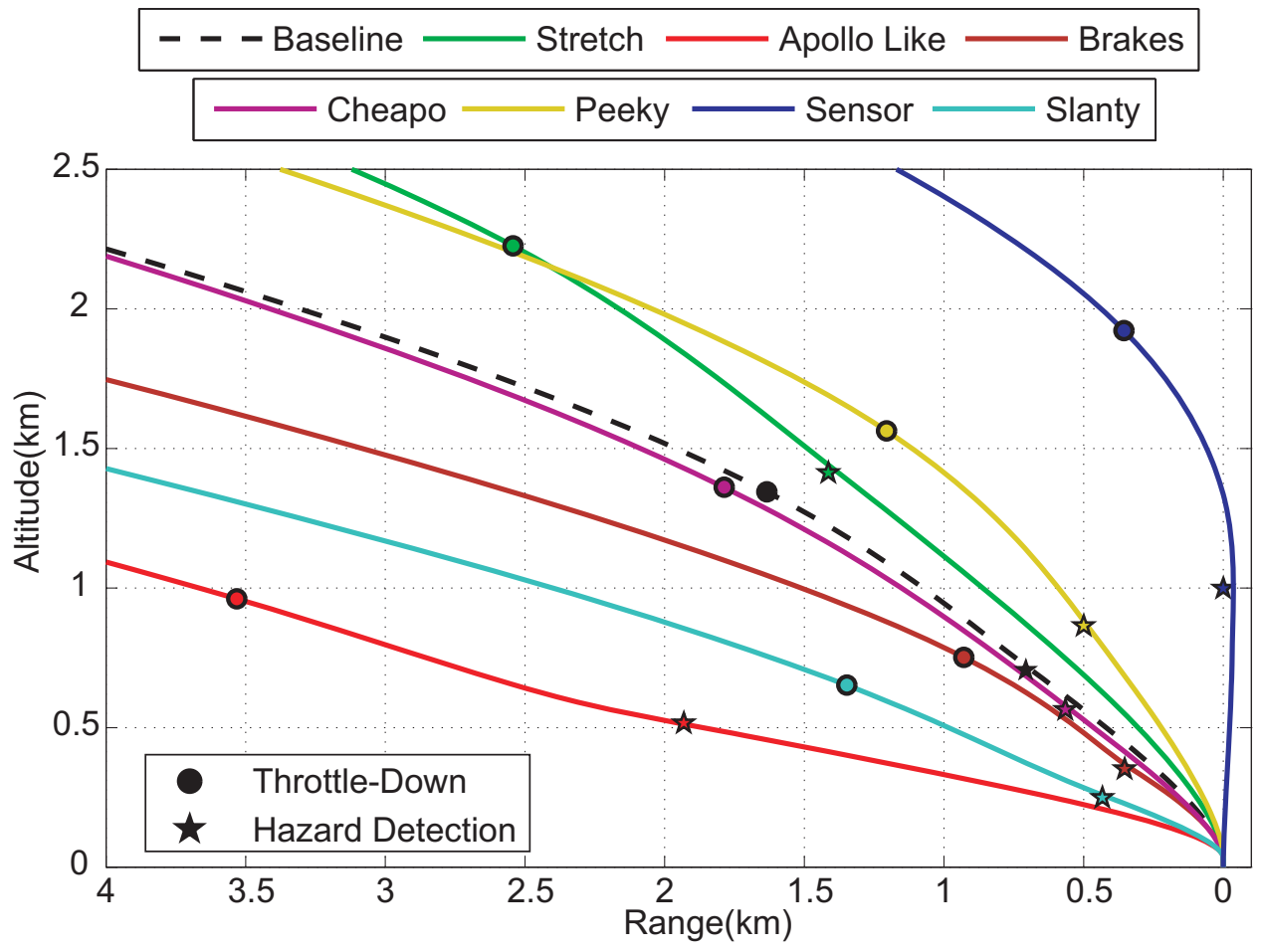

Fig. 3.3: The path of the final portion of the descent for the reduced trajectory trade space. The circles indicate where the engine throttles down and the stars indicate the point the guidance attempts to reach for HDA initiation. 
Table 3.2: Initial 1- $\boldsymbol{\sigma}$ Position/Velocity Covariance Levels for the Lander

\begin{tabular}{lccc}
\hline \hline Component & Low & Intermediate & High \\
\hline Down-Range $^{\mathrm{a}}$ & $86 \mathrm{~m}$ & $1500 \mathrm{~m}$ & $10,000 \mathrm{~m}$ \\
Cross-Track $^{\mathrm{b}}$ & $70 \mathrm{~m}$ & $200 \mathrm{~m}$ & $1000 \mathrm{~m}$ \\
Vertical $^{\mathrm{c}}$ & $10 \mathrm{~m}$ & $50 \mathrm{~m}$ & $300 \mathrm{~m}$ \\
Down-Range Rate $^{\mathrm{c}}$ & $0.0095 \mathrm{~m} / \mathrm{s}$ & $0.047 \mathrm{~m} / \mathrm{s}$ & $0.28 \mathrm{~m} / \mathrm{s}$ \\
Cross-Track Rate $^{\mathrm{b}}$ & $0.07 \mathrm{~m} / \mathrm{s}$ & $0.2 \mathrm{~m} / \mathrm{s}$ & $1.0 \mathrm{~m} / \mathrm{s}$ \\
Vertical Rate $^{\mathrm{a}}$ & $0.08 \mathrm{~m} / \mathrm{s}$ & $1.5 \mathrm{~m} / \mathrm{s}$ & $9.5 \mathrm{~m} / \mathrm{s}$ \\
\hline${ }^{\mathrm{a}}$ Correlation coef. of Down-Range and Vertical Rate: -0.9. \\
${ }^{\mathrm{b}}$ Correlation coef. of Cross-Track and Cross-Track Rate: -0.9. \\
${ }^{\mathrm{c}}$ Correlation coef. of Down-Range Rate and Vertical: -0.9.
\end{tabular}

\subsubsection{Initial Errors}

The initial covariances of the lander position and velocity are set at values that reflect various durations of Earth-based tracking and various mission scenarios. The values in Table 3.2 are based on navigation error covariances derived for the Orion Crew Exploration Vehicle and Lunar Surface Access Module [5]. The high covariance level corresponds with a scenario where the lander starts on the descent trajectory immediately after arriving in lunar orbit. Likewise, the low covariance level corresponds with scenarios where the lander loiters in a circular orbit around the moon, giving ample time for tracking. The intermediate values were used as the baseline and the effect of low initial state navigation errors were also briefly investigated.

The initial covariance of the landing site is also subject to error because of imprecisions in lunar mapping missions. For this analysis, it is assumed that maps will be available from the Lunar Reconnaissance Orbiter (LRO) that meet the LRO mission objectives [6]. The designers of the LRO expect that it will be able to survey potential landing sites with an accuracy of $100 \mathrm{~m} \mathrm{3- \sigma}$ in the north and east directions and $1 \mathrm{~m} \mathrm{3- \sigma}$ in the vertical direction. For conservativeness, $10 \mathrm{~m} \mathrm{3-} \sigma$ is used as the accuracy of the vertical component of the LRO measurements. The accuracy in the north and east directions corresponds to an error of 12 arc seconds 3- $\sigma$ in the latitude and longitude of the landing site. This causes the reference frame of the map to be both shifted and misaligned with respect to the inertial frame. This effect is termed the map-tie error. 


\subsubsection{The Lunar Environment}

Both gravitational and non-gravitational disturbances were accounted for in DAC1. Errors in the onboard gravitational model produce 60 milligals $3-\sigma$ of acceleration error with a $400 \mathrm{~km}$ correlation distance. This represents a conservative estimate of the lunar gravity uncertainty at $10 \mathrm{~km}$ altitude and is therefore even more conservative for higher altitudes. Gravity errors should not be as significant below $10 \mathrm{~km}$ altitude because noninertial sensors will be available to supplement the accelerometers. This level of uncertainty in the gravity model produces approximately $6 \mathrm{~km} \mathrm{3- \sigma}$ along-track uncertainty over the time of the final descent (approximately 1 hour). Since there are multiple well-known methods from the Apollo era that can be used to compensate for gravity errors, the baseline gravity error can be optionally reduced to $6 \mathrm{mGals} 3-\sigma$. This level of gravitational disturbance is used throughout this research. Non-gravitational accelerations due to uncoupled jet firings, crew movement, venting, and other unmodeled accelerations were accounted for as white noise and produce approximately $500 \mathrm{~m}$ of along-track error over the time of the final descent.

\subsubsection{Baseline Sensors}

The baseline sensors for the ALHAT model include an IMU, a star camera, a velocimeter and an altimeter. The use of each sensor is explained in this section along with definitions of their respective parameters. The values for these parameters are displayed in Table 3.3. These values are the same as those used in the first stage of the ALHAT work [7] and are consistent with the DAC1 assumptions for sensors and navigation. The rate at which all of these sensors are sampled in the linear covariance simulation is $0.5 \mathrm{~Hz}$.

\section{IMU}

The lander IMU is a strap-down system with three orthogonal accelerometers to measure the components of the non-gravitational acceleration of the lander and three orthogonal gyros to measure the angular velocity of the lander. The accelerometer data is only processed during maneuvers to avoid the cumulative effects of undesired accelerometer bias 
Table 3.3: Baseline Sensor Suite Parameters

\begin{tabular}{|c|c|c|c|}
\hline Sensor & Parameter & 1- $\sigma$ Steady-State Val. & Time Const. \\
\hline \multirow{4}{*}{ Accelerometers } & Bias: & 30 micro-g & $1 \times 10^{4} \mathrm{~s}$ \\
\hline & Nonorthogonality: & $20 \mathrm{arcsec} / \mathrm{axis}$ & $1 \times 10^{4} \mathrm{~s}$ \\
\hline & Scale factor: & $66 \mathrm{PPM}$ & $1 \times 10^{4} \mathrm{~s}$ \\
\hline & Random walk: & $5 \times 10^{-5} \mathrm{mps} / \sqrt{s}$ & \\
\hline \multirow{4}{*}{ Gyroscopes } & Bias: & $0.02 \mathrm{deg} / \mathrm{hr}$ & $1 \times 10^{7} \mathrm{~s}$ \\
\hline & Nonorthogonality: & $20 \operatorname{arcsec} /$ axis & $1 \times 10^{6} \mathrm{~s}$ \\
\hline & Scale factor: & 1.6 PPM & $1 \times 10^{6} \mathrm{~s}$ \\
\hline & Random walk: & $5 \times 10^{-5} \mathrm{deg} / \sqrt{s}$ & \\
\hline \multirow{2}{*}{ Star Camera } & Alignment bias: & $20 \operatorname{arcsec} /$ axis & $1 \times 10^{6} \mathrm{~s}$ \\
\hline & Noise: & $50 \operatorname{arcsec}$ & \\
\hline \multirow{6}{*}{ Altimeter } & Bias: & $0.5 \mathrm{~m}$ & $100 \mathrm{~s}$ \\
\hline & Scale Factor: & $0.1 \%$ & $100 \mathrm{~s}$ \\
\hline & Terrain Elv: & $33 \mathrm{~m}$ (range $>500 \mathrm{~m})$ & $10 \mathrm{~km}$ (distance) \\
\hline & & $3.3 \mathrm{~m}($ range $<500 \mathrm{~m})$ & $10 \mathrm{~km}$ (distance) \\
\hline & Noise: & $5 \mathrm{~m}(2-20 \mathrm{~km}$ alt $)$ & \\
\hline & & $1 \mathrm{~m}(0.01-2 \mathrm{~km}$ alt $)$ & \\
\hline \multirow{4}{*}{ Velocimeter } & Bias: & $0.001 \mathrm{~m} / \mathrm{s}$ & $100 \mathrm{~s}$ \\
\hline & Misalignment: & $0.33 \mathrm{mrad} /$ axis & $1 \times 10^{6} \mathrm{~s}$ \\
\hline & Scale Factor: & $0 \mathrm{PPM}$ & $100 \mathrm{~s}$ \\
\hline & Noise: & $0.06 \mathrm{~m} / \mathrm{s}$ & \\
\hline
\end{tabular}

and noise. The gyros operate continuously during the entire simulation. The error models of both the accelerometers and the gyros include biases and scale factors as well as nonorthogonality and random walk.

\section{Star Camera}

Because the gyroscopes in the IMU can only measure the rotation rate of the lander, a star camera is included in the baseline sensor suite to measure the lander's actual orientation based on the position of a number of guide stars. The star camera functions continuously during the entire simulation. The error model for the star camera includes a misalignment error and instrument noise.

\section{Altimeter}

The altimeter onboard the lander provides a measurement of its altitude. It operates from an altitude of $20 \mathrm{~km}$ down to the surface with varying precision as shown in Table 3.3. The model for the altimeter includes an unknown bias and scale factor, as well as 
instrument noise. The performance of the altimeter also depends on the uncertainty of the terrain elevation, which is modeled as a distance-correlated Markov process. When the lander is further than $500 \mathrm{~m}$ up-range of the landing site, the terrain elevation uncertainty is set at $100 \mathrm{~m} \mathrm{3- \sigma}$ with a $10 \mathrm{~km}$ correlation distance. When it is within $500 \mathrm{~m}$ range of the landing site this uncertainty drops to $10 \mathrm{~m} \mathrm{3- \sigma}$ to account for better knowledge of the terrain around the landing site.

\section{Velocimeter}

The velocimeter onboard the lander is a Doppler radar that provides a three-axis measurement of the velocity of the lander with respect to the surface of the moon. The velocimeter operates when the lander is between $5 \mathrm{~m}$ and $2 \mathrm{~km}$ altitude. The model of the velocimeter accounts for an unknown bias, misalignment, scale factor, and instrument noise.

\subsubsection{Reference Frames}

The following five classes of coordinate systems are used in the development of the mathematical models for the dynamics and sensors. In this paper, a coordinate transformation from frame $A$ to frame $B$ is represented by $\mathcal{T}_{A}^{B}$.

\section{Inertial Frame}

The reference frame for all other coordinate systems is the Lunar-Centered Inertial frame (LCI). It is taken to be the J2000 reference frame with its origin at the center of mass of the moon. The J2000 frame is defined via the FK5 star catalog with a standard epoch defined as 1.5 January 2000, or 12 P.M. (noon) on January $1^{\text {st }} 2000$ in the Barycentric Dynamical Time (TBD) time scale [8]. In this paper, a vector represented in this frame is denoted by a superscript $i$.

\section{Lunar Surface Fixed Frame}

The lunar surface fixed (LSF) coordinate system is a non-inertial frame that rotates and orbits with the moon with its origin centered at the landing site. The axes of this 
coordinate system are in the vertical, north, and east directions. In this paper, a vector represented in this frame is denoted by a superscript $s$. Note that the map-tie error must be accounted for in transformations between the LCI and LSF frames.

\section{Local Frame}

The local-vertical, local-horizon (LVLH) frame is a coordinate frame whose origin moves with the lander and has components that point in the down-range, vertical, and cross-track directions. It will be denoted by a superscript $L V L H$.

\section{Lander Body}

The lander body frame is centered at the center of gravity (CG) of the lander and its axes correspond to the principal axes of the lander. In this paper, a vector represented in this frame is denoted by a superscript $b$.

\section{Sensor Frames}

The coordinate system corresponding to each sensor is based on the principal axes of the sensor (e.g., axial and transverse directions). Examples include the star camera (superscript star) and the velocimeter (superscript vel) frames.

\subsection{Orbital Elements and Covariances}

Orbits are often defined by sets of 6 orbital elements $[9,10,11,12]$. In one common set, the semi-major axis and eccentricity of an ellipse are used to define the shape of the orbit. The inclination, the argument of perigee, and the right ascension of the ascending node (RAAN) then define the orientation of the orbit. Finally, the location of the spacecraft on the orbit is defined by the mean anomaly (MA).

When the covariance of a satellite is given with respect to its orbital elements, the element covariance must be transformed into the position and velocity (or state) covariance in the inertial frame before the errors can be propagated. A linear approximation to this transformation can be used quite reliably if the covariance is sufficiently small. Montenbruck 
outlines an analytic derivation of the partial derivative of a state, $\boldsymbol{x}$, with respect to the orbital elements, $\boldsymbol{\alpha}[11]$. The orbital element covariance can then be transformed into a state covariance using the formula

$$
P_{x}=\left(\frac{\partial \boldsymbol{x}}{\partial \boldsymbol{\alpha}}\right) P_{\alpha}\left(\frac{\partial \boldsymbol{x}}{\partial \boldsymbol{\alpha}}\right)^{T}
$$

\subsection{Frozen Lunar Orbits}

A "frozen" orbit [12] is designed such that at least one of its orbital elements remain constant in the presence of a perturbing force. In order to provide a reliable radio network for communication and navigation, a great deal of effort has been invested into designing frozen lunar orbits that retain constant orbital elements in the presence of both gravitational and non-gravitational disturbances. Three-body gravitational disturbances from the earth are the biggest concern for high-altitude lunar orbits, such as those necessary for an effective global lunar network with a minimal number of satellites. In cases with high inclinations, these three-body perturbations can mutate a circular orbit into a near-parabolic orbit within a few years' time $[3,13]$. Care must be taken to determine stable orbits with inclinations high enough to provide constant coverage of the poles. Todd Ely presents a method by which a highly-inclined frozen orbit can be designed such that it remains boundedly stable in the presence of both gravitational [14] and solar pressure [13] disturbances without the need for deterministic orbit maintenance.

\subsection{Terrain-Relative Navigation}

There are multiple types of terrain-relative navigation techniques that have been investigated by ALHAT [15]. All can be classified in two categories: active and passive. The active techniques use laser ranging to produce a 3-dimensional terrain map while the passive techniques use conventional CCD cameras. The simplest is a passive system that uses image processing techniques to either locate craters and other landmarks and correlate them with an onboard database or to correlate rectified images taken by the lander with lunar maps. In addition to providing a global position fix, features on the surface can then be tracked 
between frames to observe the velocity of the spacecraft relative to the surface. Depending on the accuracy of the camera, maps, and databases, when these techniques are used in conjunction with an extended Kalman filter, the position of the spacecraft can be estimated with an accuracy on the order of $40 \mathrm{~m}$ or less [16]. This method, however, lacks robustness because extreme lighting conditions can significantly change the appearance of the craters and landmarks become sparse near the lunar poles. This last effect is particularly significant because the highest priority landing site is located near the moon's southern pole.

An active TRN sensor is much more robust but requires more energy as it employs a laser scanner to illuminate points on the surface and to measure distances to those features. This laser could potentially be articulated with a fast steering mirror or simply be fixed to the frame of the lander and scan the terrain as it goes by. A third type of active sensor could use a laser flash to illuminate the surface. Because these methods offer complete measurements of the lander's position with respect to features in the terrain regardless of lighting conditions and with a reduced dependance on the type of terrain, this type of measurement is assumed in this research.

The baseline values for the parameters associated with the TRN are shown in Table 3.4. The 3-dimensional maps for the TRN algorithm will be taken from the results of the LRO mission, which will be placed in a polar orbit at an altitude of $50 \mathrm{~km}$ later in 2009 . The grid density of these maps is expected to be approximately 0.001 degrees latitude by 0.04 degrees longitude [6]. This means that the resolution of the maps will be higher at the poles than at the equator. Because the landing site is near the South Pole, the map resolution will increase as the lander approaches the landing site [15]. For this reason, the map resolution error was assumed to be $35 \mathrm{~m} \mathrm{3- \sigma}$ for surface features located more than 5 $\mathrm{km}$ from the landing site, and $2 \mathrm{~m} \mathrm{3-} \sigma$ for closer features. Although this two-tiered terrain mapping error model is simplified relative to what would be provided from surface mapping missions, it is complex enough to help understand the impact of changes in map resolution on TRN performance. The uncertainty of the longitude and latitude of the map caused by the map-tie error also has an effect on the TRN performance. 
Table 3.4: Baseline TRN Sensor Parameters

\begin{tabular}{lcc}
\hline \hline Parameter & $\begin{array}{c}\text { 1- } \boldsymbol{\sigma} \text { Steady- } \\
\text { State Value }\end{array}$ & $\begin{array}{c}\text { Time Constant/ } \\
\text { Correlation Distance }\end{array}$ \\
\hline Instrument Noise & $1 \mathrm{~m}$ & $\mathrm{~N} / \mathrm{A}$ \\
Instrument Bias & $1 \mathrm{~m}$ & $100 \mathrm{~s}$ \\
Instrument Misalignment & $0.33 \mathrm{mrad}$ & $1 \times 10^{6} \mathrm{~s}$ \\
Instrument Scale-Factor & $100 \mathrm{PPM}$ & $100 \mathrm{~s}$ \\
Map Resolution (range $>5 \mathrm{~km})$ & $11.67 \mathrm{~m}$ & $4 \mathrm{~km}$ \\
Map Resolution (range $<5 \mathrm{~km})$ & $0.67 \mathrm{~m}$ & $4 \mathrm{~km}$ \\
Map-Tie Error & 4 arcsec & invariant \\
\hline
\end{tabular}

\subsection{Radiometric Navigation}

The complete architecture of the Lunar Network (LN) as proposed by the Jet Propulsion Laboratory (JPL) consists of two LRSs in frozen lunar orbits with periods of 12 hours and two LCTs located near the landing site [17]. Each of these LN assets communicates on the S-band and would have an atomic clock onboard to ensure that the transmitted frequencies and times are as accurate as possible. At most, the clocks in the LN assets will differ by 10 nanoseconds. They will support both 1-way and 2-way range and Doppler (range-rate) measurements to up to five users (e.g., landers, rovers, and astronauts). User radios will be designed to support a single 2-way link and up to four 1-way links simultaneously [18].

The signal for a 2-way measurement originates from the LN asset, is transponded by the user, and then collected by the same asset. The asset then calculates the range of the user relative to itself using the time of flight of the signal. Due to unmodeled delays in the transponder this time-of-flight measurement is subject to a small bias and noise with a standard deviation of 35 nanoseconds, which corresponds to approximately 10.5 m 1- $\sigma$. It also determines the range rate of the user relative to itself by measuring the Doppler shift of the returned signal with an accuracy of approximately $0.5 \mathrm{~mm} / \mathrm{s} 1-\sigma$. These measurements are then transmitted to the user [18]. The values associated with 2-way radiometric measurements are summarized in Table 3.5.

The signal for a 1-way measurement also originates from the LN asset. The user must then use his own clock (which will not be as accurate as the asset's clock) to measure the time of flight and the doppler shift. Using the combined 1-way and 2-way data, the user can 
Table 3.5: Baseline Radiometric Parameters

\begin{tabular}{|c|c|c|c|}
\hline Parameter & $1-\sigma$ & Steady-State Val. & Time Const. \\
\hline Range Bias: & & $35 \mathrm{~ns}(10.5 \mathrm{~m})$ & $12 \mathrm{hr}$ \\
\hline Range Noise: & & $35 \mathrm{~ns}(10.5 \mathrm{~m})$ & $12 \mathrm{hr}$ \\
\hline Doppler Bias: & & $0.5 \mathrm{~mm} / \mathrm{s}$ & $12 \mathrm{hr}$ \\
\hline Doppler Noise: & & $0.5 \mathrm{~mm} / \mathrm{s}$ & $12 \mathrm{hr}$ \\
\hline
\end{tabular}

then resolve his time and frequency offset relative to the LN assets. For simplicity, this time and frequency offset can be ignored, and because these clock errors are the only difference between 1-way and 2-way radiometric measurement models, the 2-way measurement models can be used for all LN assets.

When working with radiometric measurements, it is important to remember their geometry. The geometry vectors of a scalar measurement are defined as the partial derivatives of the measured quantity with respect to the vectors being estimated [9]. Because a geometry vector is a partial derivative, the direction in which it points indicates the direction in which the measurement is sensitive (i.e., in which direction information about the estimated vector is available from the measurement) and its magnitude shows how the measurement's sensitivity varies with the geometry. For example, if the position of a beacon with respect to the lander is given by $\boldsymbol{r}_{b / \ell}$ and the range measurement is given by $\rho=\left|\boldsymbol{r}_{b / \ell}\right|$, then the position geometry vector, is given by

$$
\boldsymbol{h}_{r}(\rho)^{T}=\frac{\partial \rho}{\partial \boldsymbol{r}_{b / \ell}}=\left(\frac{\boldsymbol{r}_{b / \ell}}{\left|\boldsymbol{r}_{b / \ell}\right|}\right)^{T}
$$

This means that position information about the relative geometry is obtained from this measurement along the line-of-sight between the lander and the beacon. Because the magnitude of the geometry vector is always unity, the sensitivity of the measurement is independent of the relative position and velocity.

The geometry of the range-rate measurements, on the other hand, is more complicated. If the velocity of the beacon with respect to the lander is given by $\boldsymbol{v}_{b / \ell}$ and the range-rate 
measurement is given by

$$
\dot{\rho}=\left(\boldsymbol{v}_{b / \ell}\right)^{T} \frac{\boldsymbol{r}_{b / \ell}}{\left|\boldsymbol{r}_{b / \ell}\right|}
$$

then the position geometry vector is given by

$$
\boldsymbol{h}_{r}(\dot{\rho})^{T}=\frac{\partial \dot{\rho}}{\partial \boldsymbol{r}_{b / \ell}}=\left(\boldsymbol{v}_{b / \ell}\right)^{T} \frac{\left|\boldsymbol{r}_{b / \ell}\right|^{2} I_{3 \times 3}-\boldsymbol{r}_{b / \ell}\left(\boldsymbol{r}_{b / \ell}\right)^{T}}{\left|\boldsymbol{r}_{b / \ell}\right|^{3}}
$$

and the velocity geometry vector is given by

$$
\boldsymbol{h}_{v}(\dot{\rho})^{T}=\frac{\partial \dot{\rho}}{\partial \boldsymbol{v}_{b / \ell}}=\left(\frac{\boldsymbol{r}_{b / \ell}}{\left|\boldsymbol{r}_{b / \ell}\right|}\right)^{T}
$$

This means that not only is a constant level of velocity information available along the line-of-sight, but information about the relative position is also available. The sensitivity

of the position component of the range-rate measurement goes as the quantity $\left|\boldsymbol{v}_{b / \ell}\right| /\left|\boldsymbol{r}_{b / \ell}\right|$. Apparently, better position information is available from the range-rate measurement when the beacon and the lander are relatively close together and have a high relative velocity. By taking the inner product between $\boldsymbol{h}_{r}(\dot{\rho})$ and $\boldsymbol{r}_{b / \ell}$ and the inner product between $\boldsymbol{h}_{r}(\dot{\rho})$ and the cross product of $\boldsymbol{r}_{b / \ell}$ and $\boldsymbol{v}_{b / \ell}$, it can be determined that the direction in which this information is available is perpendicular to the relative position vector and in the plane defined by the relative position and velocity vectors.

\subsection{Kalman Filtering}

The Kalman filter has long been the mainstay of autonomous realtime navigation since Kalman published his original work in 1960 [19] and is now extremely well-known and welldocumented in the literature $[20,21]$. It provides an optimal method to take advantage of a priori models of the system and noisy, imperfect, asynchronous measurements from physical sensors to estimate the state of a system. In addition to the optimal estimate of the state, the filter also provides the covariance, or uncertainty, of the estimate. For the Kalman filter to function, it requires that the system be linearized about a nominal trajectory. To improve performance, the Kalman filter can be "extended" to function around the current 
estimate of the state. This extended Kalman filter (EKF) can then continue to optimally estimate the state of the system even if the state significantly deviates from the nominal trajectory. Of course, it is important to remember that even though the Kalman filter is an optimal estimator, it is only as accurate as the mathematical models of the system it uses. Therefore, care must be taking when designing and tuning a Kalman filter.

\subsection{Covariance Analysis}

An efficient alternative to Monte Carlo analysis for analyzing the performance of a dynamic system is (linear) covariance analysis. Consider the block diagram of a generic Monte Carlo analysis of a closed-loop GNC system illustrated in Fig. 3.4. The simulation for the analysis consists of truth models ${ }^{1}$ for the sensors, actuators, environment, and system dynamics, as well as flight software models for navigation (e.g. the onboard Kalman filter) and translational and rotational guidance and control. A Monte Carlo approach would require the system to run multiple times to produce the statistics of the dispersions (often called the control errors) and the navigation errors. A covariance analysis approach, on the other hand, requires each of the blocks to be approximated by a linear operation. The covariance of the true and navigation states can then be propagated through the linearized systems to find the same performance statistics, but in a single run. Because it uses linearized models of the system and assumes that all stochastic processes can be approximated by Gaussian distributions, covariance analysis may not provide the detailed, high-fidelity analysis of a complex Monte Carlo simulation. It is, however, usually accurate enough for use in the early design analysis of the system to aid in the reduction of a large trade space. In the case of traditional GNC space systems, the differences between linear covariance and Monte Carlo results are typically less than $10 \%$ [22] though results need to be periodically verified. For a complete discussion of the use of covariance analysis for GNC systems, the reader is referred to Maybeck [21] and Geller [23].

\footnotetext{
${ }^{1}$ Truth models are simulated models with which the GNC system interacts. In many cases they have higher fidelity than the navigation models.
} 


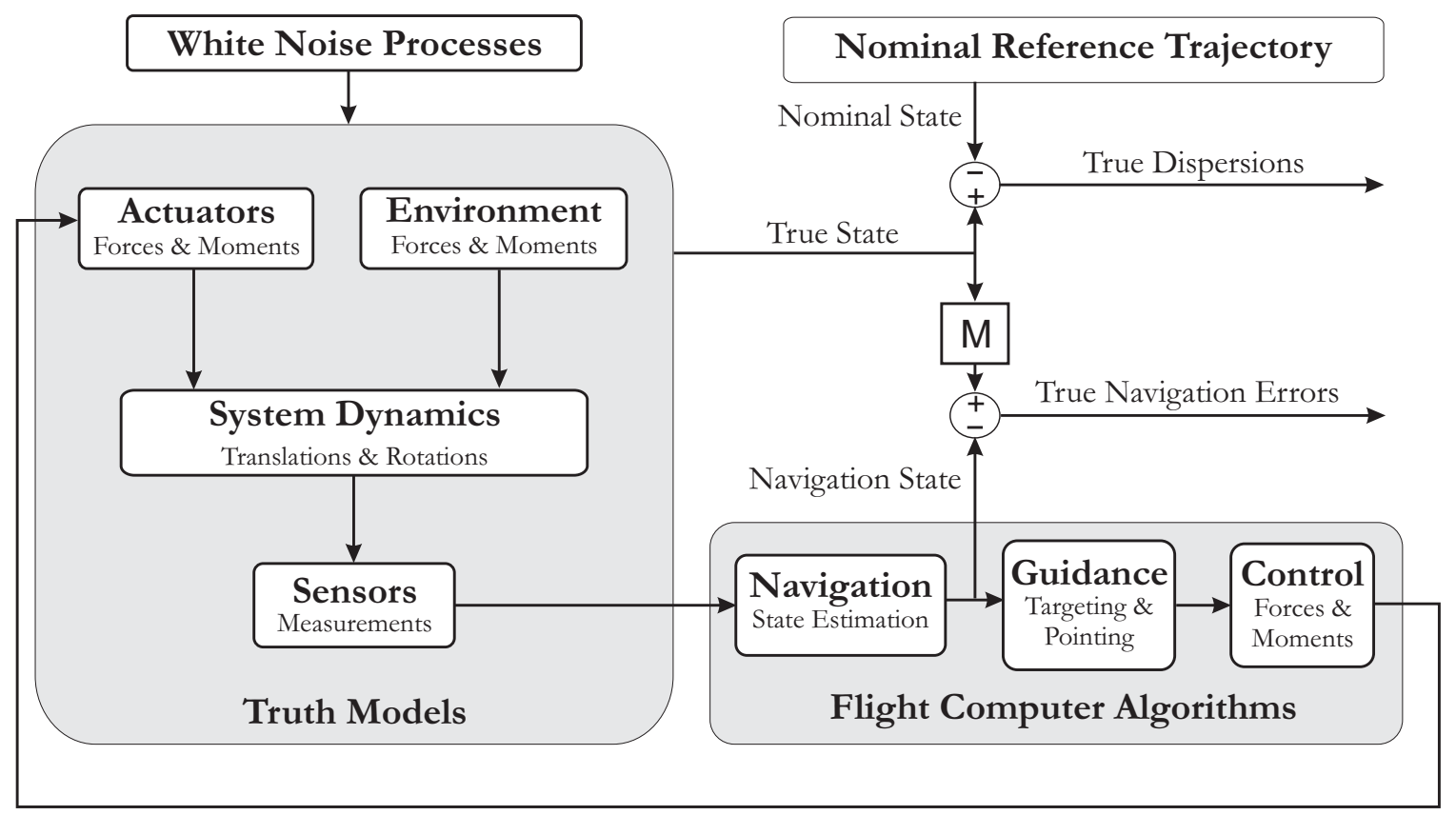

Actuator Commands

Fig. 3.4: Block diagram for a Monte Carlo analysis of a generic closed-loop GNC system 


\section{Chapter 4}

\section{Method}

\subsection{Linear Covariance Simulation}

Because of the large number of possible configurations, both phases of this study readily lent themselves to linear covariance simulation. For the first phase of this analysis, the linear covariance simulation that was used in the previous ALHAT analysis [7] was modified to include a higher-fidelity model of the TRN sensor (see page 26). This simulation now has a total of 63 true states for the lander, the landing site, the sensors, and the environment. When the full-state Kalman filter was included, this total was nearly doubled for a grand total of 123 states. The total was not completely doubled because the gyro measurements were used to replace the angular velocity states.

Eighteen states were added for the second phase of the analysis to support radiometric navigation. These include the inertial position and velocity of each LRS and biases for the range and range-rate measurements for up to three LN assets. It was assumed that the error of the LCT's position with respect to the landing site is negligible and therefore the inertial position uncertainties of the landing site and the LCT, as well as the associated position covariances, are identical. If this assumption is inappropriate for a given situation, then the navigation error of the lander relative to the landing site can be calculated by the root-sum-square of the lander's navigation error relative to the LCT and the LCT's position error relative to the landing site.

These modifications bring the number of true states to $n=81$. When these are duplicated for a nearly full-state Kalman filter, the total reaches 159 states. All 159 states are listed and identified in table 4.1 with the states modified or added for radiometric navigation italicized. The states are divided into 5 major groups: lunar lander states, LRS 
Table 4.1: List of States in the Linear Covariance Simulation

\begin{tabular}{lrl}
\hline \hline Linear Covariance States & $\#$ & Dynamics \\
\hline \hline Lander & & \\
Inertial position/velocity & 6 & Orbital motion (point mass gravity model) \\
Attitude/attitude rate & 6 & Euler's equations \\
\hline Orbiting Beacons (2) & & \\
Inertial position/velocity & 12 & Orbital motion (point mass gravity model) \\
\hline Reference Site & & \\
Landing site/LCT error & 3 & Lunar rotation \\
\hline Sensor Parameters & & \\
Accelerometers & 9 & $1^{\text {st }}$ order Markov bias, scale-factor, and misalign. \\
Gyros & 9 & $1^{\text {st }}$ order Markov bias, scale-factor, and misalign. \\
Star camera & 3 & $1^{\text {st }}$ order Markov misalign. \\
Altimeter & 2 & $1^{\text {st }}$ order Markov bias, scale-factor \\
Velocimeter & 9 & $1^{\text {st }}$ order Markov bias, scale-factor, and misalign. \\
TRN sensor & 9 & $1^{\text {st }}$ order Markov bias, scale-factor, and misalign. \\
Range to 3 beacons & 3 & $1^{\text {st }}$ order Markov bias \\
Doppler to 3 beacons & 3 & $1^{\text {st }}$ order Markov bias \\
\hline Environment & & \\
Map resolution error & 3 & $1^{\text {st }}$ order Markov bias (distance correlated) \\
Terrain elevation & 1 & $1^{\text {st }}$ order Markov bias (distance correlated) \\
Gravity model error & 3 & $1^{\text {st }}$ order Markov bias (distance correlated) \\
\hline \hline Total & $\mathbf{8 1}$ & Total with filter: 159 states \\
& &
\end{tabular}

orbiter states, reference site states, sensor parameters, and environment states:

$$
\mathbf{x}=\left(\boldsymbol{x}_{\ell}, \boldsymbol{x}_{o}, \boldsymbol{x}_{r}, \boldsymbol{p}_{s}, \boldsymbol{\varepsilon}\right)^{T} .
$$

The lunar lander states consist of the inertial position, the inertial velocity, the inertialto-lander-frame quaternion, and the inertial angular velocity of the lander in the lander-body frame:

$$
\boldsymbol{x}_{\ell}=\left(\boldsymbol{r}^{i}, \boldsymbol{v}^{i}, \boldsymbol{q}_{i}^{\ell}, \boldsymbol{\omega}^{\ell}\right)^{T} .
$$

Note that in the truth model, a 4-dimensional quaternion is used for the orientation of the lander, but in the linear covariance analysis, a 3-dimensional "modified" state is used for the orientation (see pg. 30 for explanation).

The orbiter states can be divided into the position and velocities of up to two LRSs:

$$
\boldsymbol{x}_{o}=\left(\boldsymbol{r}_{l r s 1}^{i}, \boldsymbol{v}_{l r s 1}^{i}, \boldsymbol{r}_{l r s 2}^{i}, \boldsymbol{v}_{l r s 2}^{i}\right)
$$


Because the LRSs broadcast in all directions, their attitudes are not modeled.

The reference site states refer to a location on the surface of the moon that corresponds to the landing site (or an LCT when one is available), relative to which the lander will attempt to navigate. Only three states are needed to track the covariance of one reference site position,

$$
\boldsymbol{x}_{r}=\boldsymbol{r}_{r}^{i}
$$

and care will be taken to designate when the reference site is the landing site and when it is an LCT.

Of the 82 truth states, $n_{p}=47$ are sensor parameter states. The sensor parameters, $\boldsymbol{p}_{s}$, characterize the errors of the accelerometers, gyros, star camera, altimeter, velocimeter, TRN sensor, and the lander's radiometric measurements:

$$
\boldsymbol{p}_{s}=\left(\boldsymbol{p}_{\text {accel }}, \boldsymbol{p}_{\text {gyro }}, \boldsymbol{p}_{\text {star }}, \boldsymbol{p}_{\text {alt }}, \boldsymbol{p}_{\text {vel }}, \boldsymbol{p}_{\text {trn }}, \boldsymbol{p}_{\text {radio }}\right)^{T}
$$

These parameters consist of bias errors $(b)$, scale factor errors $(f)$, and misalignment errors $(\epsilon)-$ all of which are modeled as first-order Markov processes correlated by time. The elements of each set of parameters are outlined in Table 4.1.

Finally, the $n_{\varepsilon}=7$ environment states represent limited knowledge of the lunar environment. They consist of errors in the lunar gravity model, errors in the onboard map used for the TRN, and an error in the knowledge of the terrain elevation:

$$
\varepsilon=\left(\varepsilon_{\text {grav }}^{i}, \varepsilon_{\text {map }}^{s}, \varepsilon_{e l v}\right)^{T}
$$

All are modeled as first-order Markov processes with an associated correlation distance instead of a traditional correlation time constant.

The navigation state is defined by an $\hat{n}$-dimensional vector $(\hat{n}<n)$ :

$$
\hat{\mathbf{x}}=\left(\hat{\boldsymbol{x}}_{\ell}, \hat{\boldsymbol{x}}_{o}, \hat{\boldsymbol{x}}_{r}, \hat{\boldsymbol{p}}_{s}, \hat{\boldsymbol{\varepsilon}}\right)^{T}
$$


which is nearly a copy of the true states with the exception of the inertial angular velocity of the lander ${ }^{1}$. Thus, it is composed of 10 lander states, 12 orbiting beacon states, 3 reference site states, $\hat{n}_{p}=47$ parameter states, and $\hat{n}_{\varepsilon}=7$ environment states:

$$
\begin{aligned}
\hat{\boldsymbol{x}}_{\ell} & =\left(\hat{\boldsymbol{r}}^{i}, \hat{\boldsymbol{v}}^{i}, \hat{\boldsymbol{q}}_{i}^{\ell}\right)^{T} \\
\hat{\boldsymbol{x}}_{o} & =\left(\hat{\boldsymbol{r}}_{\text {lrs } 1}^{i}, \hat{\boldsymbol{v}}_{\text {lrs } 1}^{i}, \hat{\boldsymbol{r}}_{\text {lrs } 2}^{i}, \hat{\boldsymbol{v}}_{\text {lrs } 2}^{i}\right)^{T} \\
\hat{\boldsymbol{x}}_{r} & =\hat{\boldsymbol{r}}_{r}^{i} \\
\hat{\boldsymbol{p}}_{s} & =\left(\hat{\boldsymbol{p}}_{\text {accel }}, \hat{\boldsymbol{p}}_{\text {gyro }}, \hat{\boldsymbol{p}}_{\text {star }}, \hat{\boldsymbol{p}}_{\text {alt }}, \hat{\boldsymbol{p}}_{\text {vel }}, \hat{\boldsymbol{p}}_{\text {trn }}, \hat{\boldsymbol{p}}_{\text {radio }}\right)^{T} \\
\hat{\boldsymbol{\varepsilon}} & =\left(\hat{\varepsilon}_{\text {grav }}^{i}, \hat{\boldsymbol{\varepsilon}}_{\text {map }}^{s}, \hat{\varepsilon}_{\text {elv }}\right)^{T} .
\end{aligned}
$$

\subsection{Dynamics and Environment Truth Models}

The dynamics of the true state vector are given by

$$
\begin{aligned}
\dot{\boldsymbol{r}}^{i} & =\boldsymbol{v}^{i} \\
\dot{\boldsymbol{v}}^{i} & =\boldsymbol{a}_{\text {grav }}^{i}\left(\boldsymbol{r}^{i}\right)+\boldsymbol{a}_{t h r}^{i}+\varepsilon_{\text {grav }}^{i}+\boldsymbol{w}_{a} \\
\dot{\boldsymbol{q}}_{i}^{\ell} & =\frac{1}{2} \boldsymbol{\omega}^{\ell} \otimes \boldsymbol{q}_{i}^{\ell} \\
\dot{\boldsymbol{\omega}}^{\ell} & =I_{\ell}^{-1}\left[-\boldsymbol{\omega}^{\ell} \times I_{\ell} \boldsymbol{\omega}^{\ell}+\boldsymbol{T}_{\text {thr }}^{\ell}\right]+\boldsymbol{w}_{\alpha} \\
\dot{\boldsymbol{r}}_{\text {lrs } 1,2}^{i} & =\boldsymbol{v}_{\text {lrs } 1,2}^{i} \\
\dot{\boldsymbol{v}}_{l r s 1,2}^{i} & =\boldsymbol{a}_{\text {grav }}^{i}\left(\boldsymbol{r}_{\text {lrs } 1,2}^{i}\right) \\
\dot{\boldsymbol{r}}_{r}^{i} & =\boldsymbol{\omega}_{\text {moon }}^{i} \times \boldsymbol{r}_{r}^{i} \\
\dot{p}_{j} & =-p_{j} / \tau_{j}+w_{p_{j}}, \quad j=1,2,3, \ldots, n_{p} \\
\dot{\varepsilon}_{k} & =-\frac{\varepsilon_{k}}{d_{k} /\left|\boldsymbol{v}^{i}\right|}+w_{\varepsilon_{k}}, \quad k=1,2,3, \ldots, n_{\varepsilon} .
\end{aligned}
$$

The angular velocity vector of the moon is denoted by $\boldsymbol{\omega}_{\text {moon }}^{i}$ and $I_{\ell}$ is the inertia of the lander. The acceleration due to gravity is $\boldsymbol{a}_{\text {grav }}^{i}(\cdot)$. The accelerations and torques

\footnotetext{
${ }^{1}$ The angular velocity of the lander is omitted from the filter because the attitude model operates in model replacement mode [24], where gyro measurements replace the Euler equations that would otherwise model the dynamics of the angular velocity state.
} 
from the thrusters, $\boldsymbol{a}_{t h r}^{i}$ and $\boldsymbol{T}_{t h r}^{\ell}$ respectively, are prescribed by the guidance and control laws. Unmodeled translational and rotational accelerations, $\boldsymbol{w}_{a}$ and $\boldsymbol{w}_{\alpha}$, respectively, are accounted for as process noise where

$$
\begin{aligned}
E\left[\boldsymbol{w}_{a}(t) \boldsymbol{w}_{a}^{T}\left(t^{\prime}\right)\right] & =S_{a} \delta\left(t-t^{\prime}\right) \\
E\left[\boldsymbol{w}_{\alpha}(t) \boldsymbol{w}_{\alpha}^{T}\left(t^{\prime}\right)\right] & =S_{\alpha} \delta\left(t-t^{\prime}\right)
\end{aligned}
$$

Each sensor parameter $p_{j}$ is modeled as a first-order Markov process with a time constant $\tau_{j}$ and is driven by white noise $w_{p_{j}}$ with variance

$$
E\left[w_{p_{j}}(t) w_{p_{j}}\left(t^{\prime}\right)\right]=\frac{2 \sigma_{p_{j}}^{2}}{\tau_{j}} \delta\left(t-t^{\prime}\right)
$$

Similarly, each environment state $\varepsilon_{k}$ is modeled as a first-order Markov process with an associated correlation distance $d_{k}$ and is driven by white noise $w_{\varepsilon_{k}}$ with variance

$$
E\left[w_{\varepsilon_{k}}(t) w_{\varepsilon_{k}}\left(t^{\prime}\right)\right]=\frac{2 \sigma_{\varepsilon_{k}}^{2}}{d_{k} /\left|\boldsymbol{v}^{i}\right|} \delta\left(t-t^{\prime}\right)
$$

The values for $\sigma_{p_{j}}$ and $\sigma_{\varepsilon_{k}}$ are the 1- $\sigma$ steady-state values for the associated parameters that were defined in Tables 3.3, 3.4, and 3.5.

\subsection{Sensor Truth Models}

The lander IMU is a strap-down system with three orthogonal accelerometers to measure the components of the non-gravitational accelerations of the lander, and three orthogonal gyros to measure the angular velocity of the lander. The parameters for both the accelerometer and gyro error models consist of scale-factor errors, misalignment errors, and bias errors:

$$
\boldsymbol{p}_{\text {accel }}=\left(\boldsymbol{f}_{\text {accel }}, \boldsymbol{\epsilon}_{\text {accel }}, \boldsymbol{b}_{\text {accel }}\right)^{T} \quad \text { and } \quad \boldsymbol{p}_{\text {gyro }}=\left(\boldsymbol{f}_{\text {gyro }}, \boldsymbol{\epsilon}_{\text {gyro }}, \boldsymbol{b}_{\text {gyro }}\right)^{T}
$$


In terms of these parameters, the output of the IMU in the lander frame is

$$
\begin{gathered}
\tilde{\boldsymbol{a}}_{\text {accel }}^{\ell}=\left[I_{3 \times 3}+\operatorname{Diag}\left(\boldsymbol{f}_{\text {accel }}\right)\right] \delta \mathcal{T}\left(\boldsymbol{\epsilon}_{\text {accel }}\right) \mathcal{T}_{i}^{\ell}\left(\boldsymbol{q}_{i}^{\ell}\right)\left\{\boldsymbol{a}_{\text {thr }}^{i}+\boldsymbol{w}_{a}^{i}\right\}+\boldsymbol{b}_{\text {accel }}+\boldsymbol{\eta}_{\text {accel }} \\
\tilde{\boldsymbol{\omega}}^{\ell}=\left[I_{3 \times 3}+\operatorname{Diag}\left(\boldsymbol{f}_{\text {gyro }}\right)\right] \delta \mathcal{T}\left(\boldsymbol{\epsilon}_{\text {gyro }}\right) \boldsymbol{\omega}^{\ell}+\boldsymbol{b}_{\text {gyro }}+\boldsymbol{\eta}_{\text {gyro }},
\end{gathered}
$$

where the covariances of the accelerometer noise $\left(\boldsymbol{\eta}_{\text {accel }}\right)$ and gyro noise $\left(\boldsymbol{\eta}_{\text {gyro }}\right)$ are

$$
\begin{aligned}
& E\left[\boldsymbol{\eta}_{\text {accel }}(t) \boldsymbol{\eta}_{\text {accel }}^{T}\left(t^{\prime}\right)\right]=S_{\eta_{\text {accel }} \delta\left(t-t^{\prime}\right)} \\
& E\left[\boldsymbol{\eta}_{\text {gyro }}(t) \boldsymbol{\eta}_{\text {gyro }}^{T}\left(t^{\prime}\right)\right]=S_{\eta_{\text {gyro }} \delta\left(t-t^{\prime}\right) .}
\end{aligned}
$$

The star camera is used to measure the three-axis orientation of the lander. The star camera error model has only one parameter: the 3-dimensional uncertainty in the alignment of the star camera with respect to the lander-body frame. Thus $\boldsymbol{p}_{\text {star }}=\boldsymbol{\epsilon}_{\text {star }}$. The output of the star camera is modeled as

$$
\tilde{\boldsymbol{q}}_{i}^{\text {star }}=\delta \boldsymbol{q}\left(\boldsymbol{\nu}_{\text {star }}\right) \otimes \delta \boldsymbol{q}\left(\boldsymbol{\epsilon}_{\text {star }}\right) \otimes \overline{\boldsymbol{q}}_{\ell}^{\text {star }} \otimes \boldsymbol{q}_{i}^{\ell}
$$

where $\delta \boldsymbol{q}(\cdot)$ is the quaternion associated with a small rotation, $\delta \boldsymbol{q}(\boldsymbol{\epsilon}) \approx\left(\boldsymbol{\epsilon}^{T} / 2,1\right)^{T}$, and a bar over a variable denotes that it is a design value. The covariance of the measurement noise $\boldsymbol{\nu}_{\text {star }}$ is given by

$$
E\left[\boldsymbol{\nu}_{\text {star }}\left(t_{m}\right) \boldsymbol{\nu}_{\text {star }}^{T}\left(t_{n}\right)\right]=R_{\nu_{\text {star }}} \delta_{m n}
$$

The lander altimeter measures the altitude of the lander above the lunar terrain. The altimeter error model includes a scale factor and a bias, $\boldsymbol{p}_{\text {alt }}=\left(f_{\text {alt }}, b_{\text {alt }}\right)^{T}$, as well as the terrain elevation bias $\varepsilon_{e l v}$. The altitude measurement is modeled as

$$
\tilde{h}_{\text {alt }}=f_{\text {alt }}\left\{\left|\boldsymbol{r}^{i}\right|-\rho_{\text {moon }}\right\}+b_{\text {alt }}+\varepsilon_{\text {elv }}+\nu_{\text {alt }},
$$


where $\rho_{\text {moon }}$ is the local radius of the moon and $\nu_{\text {alt }}$ represents white measurement noise with variance

$$
E\left[\nu_{\text {alt }}\left(t_{m}\right) \nu_{a l t}\left(t_{n}\right)\right]=\sigma_{\nu_{a l t}}^{2} \delta_{m n}
$$

The lander velocimeter provides a 3-dimensional measurement of the surface-relative velocity in the velocimeter frame. The parameters used in the velocimeter error model are a scale factor, a bias, and an unknown misalignment of the instrument, $\boldsymbol{p}_{\text {vel }}=\left(\boldsymbol{f}_{\text {vel }}, \boldsymbol{b}_{v e l}, \boldsymbol{\epsilon}_{v e l}\right)^{T}$. In terms of these parameters, the velocimeter model can be written as

$$
\tilde{\boldsymbol{v}}_{\text {rel }}^{\text {vel }}=\left[I_{3 \times 3}+\operatorname{Diag}\left(\boldsymbol{f}_{\text {vel }}\right)\right] \delta \mathcal{T}\left(\boldsymbol{\epsilon}_{\text {vel }}\right) \overline{\mathcal{T}}_{\ell}^{\text {vel }} \mathcal{T}_{i}^{\ell}\left(\boldsymbol{q}_{i}^{\ell}\right)\left\{\boldsymbol{v}^{i}-\boldsymbol{\omega}_{\text {moon }}^{i} \times \boldsymbol{r}^{i}\right\}+\boldsymbol{b}_{\text {vel }}+\boldsymbol{\nu}_{\text {vel }},
$$

where the covariance of the measurement noise $\boldsymbol{\nu}_{v e l}$ is given by

$$
E\left[\boldsymbol{\nu}_{v e l}\left(t_{m}\right) \boldsymbol{\nu}_{v e l}^{T}\left(t_{n}\right)\right]=R_{\nu_{v e l}} \delta_{m n}
$$

A perfect measurement from the TRN sensor would be the position of the TRN sensor with respect to the reference site in the surface frame:

$$
\boldsymbol{r}_{t r n}^{s}=\boldsymbol{r}_{t r n / r e f}^{s}=\mathcal{T}_{i}^{s}\left(\boldsymbol{r}^{i}+\mathcal{T}_{\ell}^{i} \boldsymbol{r}_{t r n / c g}^{\ell}-\boldsymbol{r}_{r}^{i}\right)
$$

where $\boldsymbol{r}_{t r n / c g}^{\ell}$ is the position of the TRN with respect to the lander's center of gravity in the lander-body frame. The actual output of the TRN sensor is modeled to include errors due to instrument bias, scale-factor, and misalignment, $\boldsymbol{p}_{t r n}=\left(\boldsymbol{b}_{t r n}, \boldsymbol{f}_{t r n}, \boldsymbol{\epsilon}_{t r n}\right)^{T}$, as well as instrument noise $\left(\boldsymbol{\nu}_{t r n}\right)$, attitude dispersion $\left(\delta \boldsymbol{\theta}_{\boldsymbol{i}}^{\boldsymbol{\ell}}\right)$, map error in the surface frame, $\left(\boldsymbol{\varepsilon}_{\text {map }}^{s}\right)$, and map-tie error $(\boldsymbol{\delta} \phi)$. The actual measurement model can be written as

$$
\tilde{\boldsymbol{r}}_{t r n}^{s}=\left[I_{3 \times 3}+\operatorname{Diag}\left(\boldsymbol{f}_{t r n}\right)\right] \mathcal{T}_{i}^{s}(\boldsymbol{\delta} \boldsymbol{\phi})\left(\boldsymbol{r}_{t r n / r e f}^{s}\right)+\boldsymbol{\varepsilon}_{\text {map }}^{s}+\boldsymbol{b}_{t r n}+\boldsymbol{\nu}_{t r n}+\boldsymbol{e}\left(\delta \boldsymbol{\theta}_{i}^{\ell}, \boldsymbol{\epsilon}_{t r n}\right),
$$

where the instrument noise $\boldsymbol{\nu}_{t r n}$ has a covariance of

$$
E\left[\boldsymbol{\nu}_{t r n}\left(t_{m}\right) \boldsymbol{\nu}_{t r n}\left(t_{n}\right)^{T}\right]=R_{\nu_{t r n}} \delta_{m n}
$$




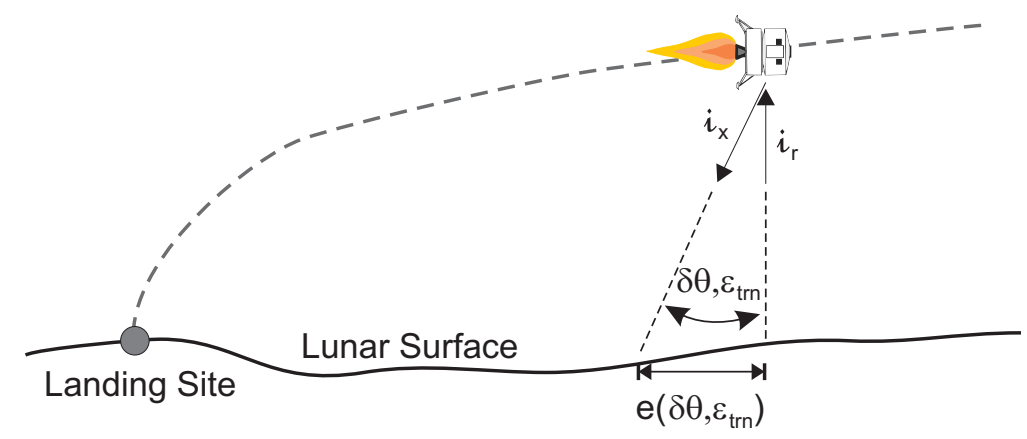

Fig. 4.1: Effect of attitude uncertainty and instrument misalignment on TRN output.

The map-tie error, which is a function of the inertial uncertainty of the reference site location:

$$
\boldsymbol{\delta} \phi=\frac{\boldsymbol{r}_{r}^{i} \times \delta \boldsymbol{r}_{r}^{i}}{\left|\boldsymbol{r}_{r}^{i}\right|^{2}}
$$

introduces an uncertainty into the transformation from the inertial frame to the surface frame: $\mathcal{T}_{i}^{s}(\boldsymbol{\delta} \phi)=\delta \mathcal{T}(\boldsymbol{\delta} \phi) \overline{\mathcal{T}}_{i}{ }^{s}$. The error in the TRN measurement caused by the misalignment of the sensor and uncertainties in the attitude of the spacecraft is given by

$$
\boldsymbol{e}\left(\delta \boldsymbol{\theta}_{i}^{\ell}, \boldsymbol{\epsilon}_{t r n}\right)=h \mathcal{T}_{i}^{s}(\boldsymbol{\delta} \phi)\left[\overline{\mathcal{T}}_{\ell}^{i} \delta \mathcal{T}\left(-\delta \boldsymbol{\theta}_{i}^{\ell}\right) \overline{\mathcal{T}}_{t r n}^{\ell} \delta \mathcal{T}\left(\boldsymbol{\epsilon}_{t r n}\right) \boldsymbol{i}_{x}^{t r n}+\boldsymbol{i}_{r}^{i}\right]
$$

This error is also illustrated in Fig. 4.1. Here, $h$ is the altitude of the lander, $\boldsymbol{i}_{x}^{t r n}$ is the unit vector along the bore-sight (x-axis) of the TRN instrument in the TRN frame, and $\boldsymbol{i}_{r}$ is the unit vector from the center of the moon to the spacecraft. It is assumed that the desired direction of the TRN bore-site is nadir along $-\boldsymbol{i}_{r}^{i}$.

For the radiometric measurements, it is assumed that 2-way range and Doppler (rangerate) measurements will be received from all surface and orbiting assets that are available and visible. The parameters associated with the radiometric measurements are range bias and Doppler bias for each beacon:

$$
\boldsymbol{p}_{\text {radio }}=\left(b_{\text {range }, \text { rrs } 1}, b_{\text {doppler }, \text { rrs } 1}, b_{\text {range }, \text { lrs } 2}, b_{\text {doppler }, \text { lrs } 2}, b_{\text {range }, l c t}, b_{\text {doppler }, l c t}\right)^{T} .
$$


A generic model for the range measurements is given by

$$
\rho_{\text {lrs }, l c t}=\left|\boldsymbol{r}^{i}-\boldsymbol{r}_{\text {lrs }, l c t}^{i}\right|+b_{\text {range }}+\nu_{\text {range }},
$$

and a generic model for the Doppler measurements is

$$
\dot{\rho}_{l r s, l c t}=\frac{\left(\boldsymbol{r}^{i}-\boldsymbol{r}_{l r s, l c t}^{i}\right)^{T}}{\left|\boldsymbol{r}^{i}-\boldsymbol{r}_{l r s, l c t}^{i}\right|}\left(\boldsymbol{v}^{i}-\boldsymbol{v}_{l r s, l c t}^{i}\right)+b_{\text {doppler }}+\nu_{\text {doppler }}
$$

where covariances for the noise terms are given by

$$
\begin{gathered}
E\left[\boldsymbol{\nu}_{\text {range }}\left(t_{m}\right) \boldsymbol{\nu}_{\text {range }}\left(t_{n}\right)^{T}\right]=R_{\nu_{\text {range }} \delta_{m n}} \\
E\left[\boldsymbol{\nu}_{\text {doppler }}\left(t_{m}\right) \boldsymbol{\nu}_{\text {doppler }}\left(t_{n}\right)^{T}\right]=R_{\nu_{\text {doppler }}} \delta_{m n} .
\end{gathered}
$$

It is assumed that radiometric measurements from the LN assets will not be hindered by outages and multi-path issues whenever the relative geometry meets certain constraints for visibility. The nominal constraint for the measurements from the LRSs is such that the LRS must be more than 10 degrees above the lander's horizon. This corresponds to the angle $\alpha$ in Fig. 4.2. This angle is calculated by solving the following set of equations:

$$
\begin{aligned}
\cos (\alpha+\gamma) & =\frac{\left(\boldsymbol{r}^{i}\right)^{T}\left(\boldsymbol{r}^{i}-\boldsymbol{r}_{l r s}^{i}\right)}{\left|\boldsymbol{r}^{i}\right|\left|\boldsymbol{r}^{i}-\boldsymbol{r}_{l r s}^{i}\right|} \\
\sin (\gamma) & =\frac{\rho_{\text {moon }}}{\left|\boldsymbol{r}^{i}\right|}
\end{aligned}
$$

where $\gamma$ is defined in the figure and $\rho_{\text {moon }}$ is the radius of the moon. The constraint for the measurements from the LCT is such that the lander must be more than 10 degrees above the LCT's horizon. This corresponds to the angle $\beta$ in the figure, which can be calculated by solving the following equation:

$$
\cos \left(\beta+90^{\circ}\right)=\frac{\left(\boldsymbol{r}_{l c t}^{i}\right)^{T}\left(\boldsymbol{r}_{l c t}^{i}-\boldsymbol{r}^{i}\right)}{\left|\boldsymbol{r}_{l c t}^{i}\right|\left|\boldsymbol{r}_{l c t}^{i}-\boldsymbol{r}^{i}\right|}
$$




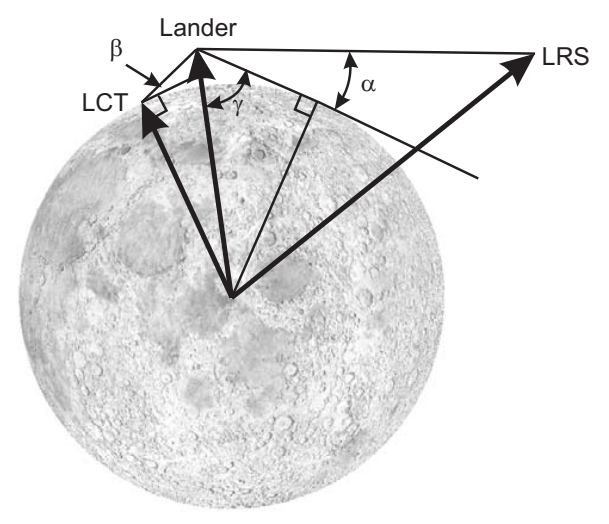

Fig. 4.2: In order for measurements to be received from an LRS or LCT, the angle $\alpha$ or $\beta$ must be greater than the elevation constraint.

\subsection{Navigation Algorithm}

The propagation equations for the navigation state are based upon the truth models of the dynamics and environment:

$$
\begin{aligned}
\dot{\hat{\boldsymbol{r}}}^{i} & =\hat{\boldsymbol{v}}^{i} \\
\dot{\hat{\boldsymbol{v}}}^{i} & =\boldsymbol{a}_{\text {grav }}^{i}\left(\hat{\boldsymbol{r}}^{i}\right)+\hat{\boldsymbol{\varepsilon}}_{\text {grav }}^{i}+\hat{\boldsymbol{a}}_{\text {nongrav }}^{i}\left(\tilde{\boldsymbol{a}}_{\text {accel }}^{\ell}, \hat{\boldsymbol{p}}_{\text {accel }}\right) \\
\dot{\hat{\boldsymbol{q}}}_{i}^{\ell} & =\frac{1}{2} \hat{\boldsymbol{\omega}}_{\text {comp }}\left(\tilde{\boldsymbol{\omega}}^{\ell}, \hat{\boldsymbol{p}}_{\text {gyro }}\right) \otimes \hat{\boldsymbol{q}}_{i}^{\ell} \\
\dot{\boldsymbol{r}}_{\text {lrs } 1,2}^{i} & =\hat{\boldsymbol{v}}_{\text {lrs } 1,2}^{i} \\
\dot{\hat{\boldsymbol{v}}}_{l r s 1,2}^{i} & =\boldsymbol{a}_{\text {grav }}^{i}\left(\hat{\boldsymbol{r}}_{\text {lrs } 1,2}^{i}\right) \\
\dot{\hat{\boldsymbol{r}}}_{r}^{i} & =\boldsymbol{\omega}_{m o o n} \times \hat{\boldsymbol{r}}_{o}^{i} \\
\dot{\hat{p}}_{k} & =-\left(\hat{p}_{k} / \hat{\tau}_{k}\right), \quad k=1,2,3, \ldots, \hat{n}_{p} \\
\dot{\hat{\varepsilon}}_{k} & =-\frac{\hat{\varepsilon} \hat{\varepsilon}_{k}}{\hat{d}_{k} /\left|\hat{\boldsymbol{v}}^{i}\right|}, \quad k=1,2,3, \ldots, n_{\varepsilon} .
\end{aligned}
$$

The accelerometers' measurement of the accelerations caused by the thrusters and nongravitational disturbances, $\tilde{\boldsymbol{a}}_{\text {accel }}^{\ell}$, is corrected using the best estimates of the error model parameters and is used directly in the propagation of the lander's position and velocity:

$$
\hat{\boldsymbol{a}}_{\text {nongrav }}^{i}\left(\tilde{\boldsymbol{a}}_{\text {accel }}^{\ell}, \hat{\boldsymbol{p}}_{\text {accel }}\right)=\mathcal{T}_{\ell}^{i}\left(\hat{\boldsymbol{q}}_{\ell}^{i}\right) \delta \mathcal{T}\left(-\hat{\boldsymbol{\epsilon}}_{\text {accel }}\right)\left\{\left[I_{3 \times 3}-\operatorname{Diag}\left(\hat{\boldsymbol{f}}_{\text {accel }}\right)\right] \tilde{\boldsymbol{a}}_{\text {accel }}^{\ell}-\hat{\boldsymbol{b}}_{\text {accel }}\right\}
$$


Similarly, the corrected measurement of the angular velocity is used to propagate the quaternion of the lander:

$$
\hat{\boldsymbol{\omega}}_{\text {comp }}\left(\tilde{\boldsymbol{\omega}}^{\ell}, \hat{\boldsymbol{p}}_{\text {gyro }}\right)=\delta \mathcal{T}\left(-\hat{\boldsymbol{\epsilon}}_{\text {gyro }}\right)\left\{\left[I_{3 \times 3}-\operatorname{Diag}\left(\hat{\boldsymbol{f}}_{\text {gyro }}\right)\right] \tilde{\boldsymbol{w}}_{\text {gyro }}^{\ell}-\hat{\boldsymbol{b}}_{\text {gyro }}\right\} .
$$

These propagation equations can be written in the compact form

$$
\dot{\hat{\mathbf{x}}}=\hat{\boldsymbol{f}}(\hat{\mathbf{x}}, \tilde{\boldsymbol{y}}, t)
$$

where $\tilde{\boldsymbol{y}}=\left(\tilde{\boldsymbol{a}}_{\text {accel }}^{\ell}, \tilde{\boldsymbol{w}}_{\text {gyro }}^{\ell}\right)^{T}$ is the output of the IMU [see Equations (4.27) and (4.28) on page 25]. Linearizing this system of equations leads to the navigation state covariance propagation equations

$$
\dot{\hat{P}}=\hat{F}_{\hat{x}} \hat{P}+\hat{P} \hat{F}_{\hat{x}}+\hat{S}_{\eta}+\hat{S}_{w}
$$

where $\hat{F}_{\hat{x}}$ is the partial of $\hat{\boldsymbol{f}}$ with respect to $\hat{\mathbf{x}}$. This partial derivative, the state process noise covariance $\hat{S}_{w}$, and the covariance $S_{\eta}$ due to gyro and accelerometer noise are given in Appendix A. This is the first of two key equations in the linear covariance analysis and is used to propagate the errors between measurements.

Note that "modified" states [25] were used in deriving the covariance propagation equation. The 4 -dimensional quaternion state, $\hat{\boldsymbol{q}}_{i}^{\ell}$, is replaced by a 3 -dimensional rotation vector state, $\hat{\boldsymbol{\theta}}_{i}^{\ell}$, and the quaternion kinematics are replaced by the Bortz equation [24]. Thus, quaternion errors $\delta \hat{\boldsymbol{q}}_{i}^{\ell}$ are replaced by a 3 -dimensional small-angle rotation vector $\delta \hat{\boldsymbol{\theta}}_{i}^{\ell}$ and the linearized Bortz equation is used to derive the attitude covariance propagation equations. This replacement facilitates linearization and helps to ensure that the covariance associated with the attitude remains well conditioned.

All the navigation state update equations

$$
\delta \hat{\mathbf{x}}_{m}=\hat{K}\left(t_{m}\right)\left[\tilde{\boldsymbol{z}}_{m}-\hat{\boldsymbol{h}}\left(\hat{\mathbf{x}}_{m}, t_{m}\right)\right]
$$


are additive:

$$
\hat{\mathbf{x}}_{m}^{+}=\hat{\mathbf{x}}_{m}^{-}+\delta \hat{\mathbf{x}}_{m}
$$

with the exception of the attitude updates, which are implemented as small quaternion rotations,

$$
\left(\hat{\boldsymbol{q}}_{i}^{\ell}\right)^{+}=\delta \boldsymbol{q}\left(\hat{\delta}_{i}^{\ell}\right) \otimes\left(\hat{\boldsymbol{q}}_{i}^{\ell}\right)^{-}
$$

When processing altimeter, velocimeter, TRN, and radiometric range and Doppler measurements, $\tilde{\boldsymbol{z}}_{m}$ is simply the output of the sensor and $\hat{\boldsymbol{h}}\left(\hat{\boldsymbol{x}}_{m}, t_{m}\right)$ is the estimate of the measurement based on the error models given in Equations (4.33), (4.35), (4.38), (4.43), and (4.44), respectively (see pages $25-28)$. When processing star camera data, $\tilde{\boldsymbol{z}}_{m}$ is a derived measurement [24] calculated from

$$
\left(\begin{array}{c}
\frac{1}{2} \tilde{\boldsymbol{z}}_{m} \\
1
\end{array}\right)=\tilde{\boldsymbol{q}}_{i}^{\text {star }} \otimes \hat{\boldsymbol{q}}_{\ell}^{i} \otimes \overline{\boldsymbol{q}}_{\text {star }}^{\ell} \otimes\left(\begin{array}{c}
-\frac{1}{2} \hat{\boldsymbol{\epsilon}}_{\text {star }} \\
1
\end{array}\right)
$$

For an extended Kalman filter, the flight computer estimate of the derived star camera measurement is

$$
\hat{\boldsymbol{h}}\left(\hat{\boldsymbol{x}}_{m}, t_{m}\right)=\hat{\mathcal{T}}_{\ell}^{\text {star }} \delta \hat{\boldsymbol{\theta}}_{i}^{\ell}+\hat{\boldsymbol{\epsilon}}_{\text {star }},
$$

where $\hat{\boldsymbol{\epsilon}}_{\text {star }}$ is the flight computer's estimate of the star camera misalignment.

The navigation state covariance update equation [20] is given by

$$
\hat{P}\left(t_{m}^{+}\right)=\left[I-\hat{K}\left(t_{m}\right) \hat{H}_{\hat{x}}\left(t_{m}\right)\right] \hat{P}\left(t_{m}^{-}\right)\left[I-\hat{K}\left(t_{m}\right) \hat{H}_{\hat{x}}\left(t_{m}\right)\right]^{T}+\hat{K}\left(t_{m}\right) \hat{R}_{\nu}\left(t_{m}\right) \hat{K}^{T}\left(t_{m}\right),
$$

where the Kalman gain $\hat{K}\left(t_{m}\right)$ is given by

$$
\hat{K}\left(t_{m}\right)=\hat{P}\left(t_{m}^{-}\right) \hat{H}_{\hat{x}}^{T}\left(t_{m}\right)\left[\hat{H}_{\hat{x}}\left(t_{m}\right) \hat{P}\left(t_{m}^{-}\right) \hat{H}_{\hat{x}}^{T}\left(t_{m}\right)+\hat{R}_{\nu}\left(t_{m}\right)\right]^{-1} .
$$

The measurement sensitivity matrices $\hat{H}_{\hat{x}}$ and the measurement covariance matrices $\hat{R}_{\nu}$ for the star camera, altimeter, velocimeter, TRN, and radiometric measurements are given in Appendix A. The covariance update equation (4.67) is the second of two key linear 
covariance analysis equations and is used to update the navigation state when measurements are available.

\subsection{Metrics}

The performance metrics for this research are conservative estimates of the 3- $\sigma$ navigation error given by

$$
p_{\text {nav }}^{i, r e l}=3 \sqrt{\operatorname{tr}\left(\hat{P}^{i, r e l}\right)}
$$

where $\hat{P}^{i, r e l}$ is either the covariance of the inertial navigation error of the lander, or the covariance of the navigation error of the lander relative to the reference site. The inertial and relative error vectors can be described using two linear operations, $A_{i}$ and $A_{\text {rel }}$ :

$$
\boldsymbol{e}_{n a v}^{i}=A_{i}(\mathbf{x}-\hat{\mathbf{x}}) \quad \text { and } \quad \boldsymbol{e}_{n a v}^{r e l}=A_{r e l}(\mathbf{x}-\hat{\mathbf{x}})
$$

These same two linear operators can be used to calculate the covariances required by Equation (4.69) using the navigation state covariance that is propagated by Equation (4.61) and updated by Equation (4.67):

$$
\hat{P}^{i}=A_{i} \hat{P} A_{i}^{T} \quad \text { and } \quad \hat{P}^{r e l}=A_{r e l} \hat{P} A_{r e l}^{T} .
$$

The most important of the two performance metrics is the relative navigation performance because it is used to assess how well the lander can navigate its way to the landing site. A useful fact about the performance metrics defined in Equation (4.69) is that because the

trace of a matrix is equal to the sum of its eigenvalues, $p_{\text {nav }}^{i, r e l}$ remains constant through coordinate transformations and therefore provides a robust measurement of the navigation performance.

\subsection{Requirements}

As stated in the introduction, the minimum requirement for a precision landing is that the navigation accuracy must be at most $100 \mathrm{~m} \mathrm{3- \sigma}$ at landing. The hazard-relative navi- 
Table 4.2: Navigation Requirements

\begin{tabular}{lr}
\hline \hline Phase & Requirement \\
\hline PDI & $1000 \mathrm{~m}$ \\
Throttle-down & $200 \mathrm{~m}$ \\
HDA & $100 \mathrm{~m}$ \\
Landing & $100 \mathrm{~m}$ \\
\hline
\end{tabular}

gation software requires the navigation accuracy to remain constant from HDA to landing. It is foreseeable that navigation requirements will also be needed at PDI and throttle-down to reduce the fuel that is used during powered descent. Otherwise, a significant amount of fuel would be required to reduce large dispersions late in the trajectory when it would have been much more efficient to make small velocity corrections earlier in the trajectory. For this reason, the navigation accuracy is required to be within $1000 \mathrm{~m}$ at PDI and $200 \mathrm{~m}$ at throttle-down. These requirements are summarized in Table 4.2.

To illustrate the motivation for TRN and radiometric navigation, the navigation error that results from only using the baseline sensors during the descent is plotted in Fig. 4.3. The lander navigation errors are initialized at the intermediate level given in Table 3.2 (page 8). After the deorbit burn, the combined effect of uncertainties in the initial state of the lander, the lunar environment, and the accelerometer model causes the navigation error to

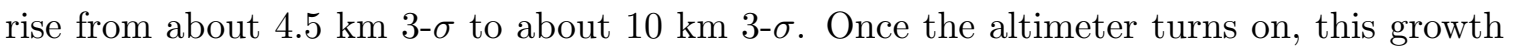
is very nearly eliminated due to correlations in the LVLH frame (see the footnotes of Table 3.2 on page 8 ). The Kalman filter is then able to use additional measurements from the altimeter to even further reduce the navigation error. The error at PDI is shown by a large

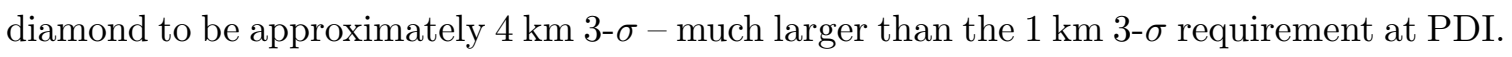
The powered descent burn itself allows the Kalman filter to use altimeter and accelerometer measurements to reduce the navigation errors to about $1.2 \mathrm{~km} 3-\sigma$ where the velocimeter turns on and the navigation error is again sharply reduced to about $700 \mathrm{~m} \mathrm{3- \sigma}$ just in time for throttle-down and HDA. This does not support either of these phases. By the time of landing, the navigation errors are only reduced to $559 \mathrm{~m} \mathrm{3-} \sigma$. Clearly, the baseline sensor suite is not able to support any of the phases required for a precision landing. 


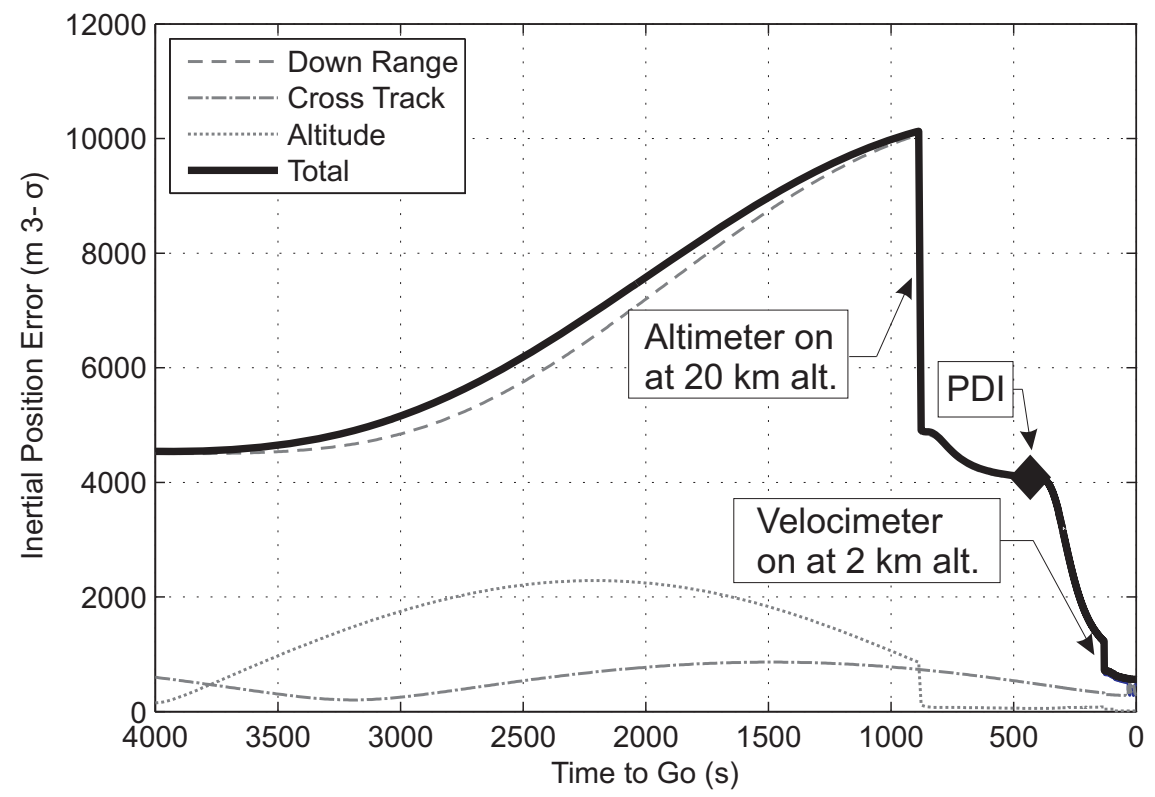

Fig. 4.3: Navigation errors resulting from only using the baseline sensors. 


\section{Chapter 5 \\ Results}

\subsection{Terrain-Relative Navigation Studies}

The objective of the linear covariance TRN analysis is to evaluate the ability of TRN to meet the navigation requirements. Particular interest is in the effect of the operating range of the TRN sensor as well as the trajectory type, the map resolution, and the sensor rate.

\subsubsection{TRN Operating Range Study}

It can easily be determined that the best navigation results would be obtained by using the TRN sensor as long as possible. There are, however, two significant reasons why the operating range of the TRN might be limited. First, the TRN sensor will likely be designed to scan the terrain directly under the lander for a particular limited range of altitudes. Second, the computer onboard the lander would be required to hold detailed maps of all the terrain over which the lander could possibly fly. This is why determining the sensitivity of the navigation accuracy to the operating range of the TRN sensor was the primary goal of this phase of the research.

To accomplish this, the time from PDI to throttle-down was divided into five equal periods. The beginning of each period was considered as a possible time for the TRN sensor to turn on and the end of each period was considered as a possible time for the sensor to turn off. The navigation accuracies resulting from the use of each possible combination of on-times and off-times was predicted using the linear covariance simulation. This gives 15 possible ranges defined by six evenly-spaced times: T1 through T6, where T1 is PDI and T6 is throttle-down. The altitude and range of the lander along the baseline trajectory and 
the navigation accuracy associated with each operating range is shown in Fig. 5.1 in which the time of PDI is defined as 0 seconds.

The same results are also displayed graphically in Fig. 5.2. In all four plots, each line represents the results from a different TRN operating range in which either the time for turning the TRN sensor on or off is varied. The top-left plot reflects the first row of the table where the initiation time is held fixed at PDI (T1) and the top-right plot reflects the second row where the initiation time is held at T2. The lower-left plot reflects the fourth column where the TRN cutoff time is held at T5 and the lower-right plot reflects the final column where the cutoff time is held at throttle-down (T6). This figure shows that a single TRN measurement reduces the navigation error from $4 \mathrm{~km}$ to about $50 \mathrm{~m}$. Once the TRN is turned off, the error growth resumes due to disturbances and residual velocity errors until the velocimeter turns on and corrects the velocity errors. Fig. 5.2 effectively shows that the time at which the TRN sensor is turned off has a much more significant effect than the time at which it turns on and that later TRN cutoff times provide better navigation performance. The effect of TRN measurements inside the area where higher resolution maps are available is also quite apparent. When these high-resolution measurements are available, the navigation errors are reduced to $10 \mathrm{~m}$ or less.

Because the baseline sensor suite can only reduce navigation errors to approximately $4 \mathrm{~km} \mathrm{3- \sigma}$ at PDI, the TRN must initiate at or before T1 to support the $1000 \mathrm{~m} \mathrm{3}-\sigma$ requirement at PDI. This means that the possible TRN operating ranges are limited to those displayed in the first row of the table in Fig. 5.1 and the top-left plot in Fig. 5.2. In this case, if the TRN design requires the TRN to operate only over a short range, it would be unlikely that TRN measurements would be available inside the area with high-resolution maps. This may not be an issue because all of the possible TRN operating ranges meet the $100 \mathrm{~m} \mathrm{3- \sigma}$ requirement. If, however, the high precision gained by TRN measurements in the high-resolution map range is desirable, then some other method would be required to support PDI. 


\begin{tabular}{|c|c|c|c|c|c|c|}
\hline & \multicolumn{5}{|c|}{ Possible TRN Cutoff Times } \\
\hline & & $\begin{array}{l}\text { T2 }=70 \mathrm{~s} \\
\mathrm{~h}=14 \mathrm{~km} \\
\mathrm{r}=200 \mathrm{~km}\end{array}$ & $\begin{array}{l}\text { T3 }=140 \mathrm{~s} \\
\mathrm{~h}=12.2 \mathrm{~km} \\
\mathrm{r}=100 \mathrm{~km}\end{array}$ & $\begin{array}{l}\text { T4 }=210 \mathrm{~s} \\
\mathrm{~h}=8.6 \mathrm{~km} \\
\mathrm{r}=50 \mathrm{~km}\end{array}$ & $\begin{array}{l}\text { T5 }=280 \mathrm{~s} \\
\mathrm{~h}=4.1 \mathrm{~km} \\
\mathrm{r}=10 \mathrm{~km}\end{array}$ & $\begin{array}{l}\text { T6 }=350 \mathrm{~s} \\
\mathrm{~h}=0.7 \mathrm{~km} \\
\mathrm{r}=0.4 \mathrm{~km}\end{array}$ \\
\hline \multirow{15}{*}{ 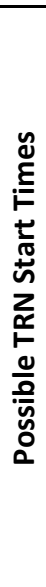 } & $\mathrm{T} 1=0 \mathrm{~s}$ & $30.2 @ \mathrm{CO}$ & $28.3 @$ CO & $26.5 @$ CO & $28.2 @$ CO & $6.3 @ \mathrm{CO}$ \\
\hline & $\mathrm{h}=14.5 \mathrm{~km}$ & $56.9 @$ TD & $42.8 @$ TD & $31.9 @$ TD & $25.4 @ T D$ & $6.3 @$ TD \\
\hline & $r=300 \mathrm{~km}$ & 44.8 @ L & $33.2 @ \mathrm{~L}$ & $24.2 @ \mathrm{~L}$ & $20.0 @$ L & $7.8 @$ L \\
\hline & $\mathrm{T} 2=70 \mathrm{~s}$ & & $29.2 @$ CO & $29.2 @$ CO & $28.0 @$ CO & $6.6 @$ CO \\
\hline & $\mathrm{h}=14 \mathrm{~km}$ & & $44.9 @ T D$ & $34.2 @$ TD & $25.6 @ T D$ & $6.6 @ T D$ \\
\hline & $r=200 \mathrm{~km}$ & & $44.9 @$ L & $26.3 @ \mathrm{~L}$ & $20.6 @ \mathrm{~L}$ & $8.1 @$ L \\
\hline & $\mathrm{T} 3=140 \mathrm{~s}$ & & & $30.0 @$ CO & $31.7 @ \mathrm{CO}$ & $6.4 @$ CO \\
\hline & $\mathrm{h}=12.2 \mathrm{~km}$ & & & $35.1 @$ @D & $26.7 @ T D$ & $6.4 @ T D$ \\
\hline & $\mathrm{r}=100 \mathrm{~km}$ & & & $27.6 @ \mathrm{~L}$ & $21.7 @ \mathrm{~L}$ & $7.9 @$ L \\
\hline & $\mathrm{T4}=210 \mathrm{~s}$ & & & & $32.3 @ \mathrm{CO}$ & $6.8 @ \mathrm{CO}$ \\
\hline & $\mathrm{h}=14 \mathrm{~km}$ & & & & $28.7 @ T D$ & $6.8 @$ TD \\
\hline & $\mathrm{r}=50 \mathrm{~km}$ & & & & $25.2 @ \mathrm{~L}$ & $8.1 @ \mathrm{~L}$ \\
\hline & $\mathrm{T5}=280 \mathrm{~s}$ & & \multirow{3}{*}{\multicolumn{2}{|c|}{$\begin{array}{ll}\text { CO: } & \text { TRN Cutoff } \\
\text { TD: } & \text { Throttle-down } \\
\text { L: } & \text { Landing }\end{array}$}} & & $6.6 @ \mathrm{CO}$ \\
\hline & $\mathrm{h}=4.1 \mathrm{~km}$ & & & & & $6.6 @$ TD \\
\hline & $r=10 \mathrm{~km}$ & & & & & $8.0 @$ L \\
\hline
\end{tabular}

Fig. 5.1: Possible TRN operating ranges on the baseline trajectory. Each cell shows the navigation performance at TRN cutoff (CO), throttle-down (TD), and landing (L). Values are in meters $3-\boldsymbol{\sigma}$.
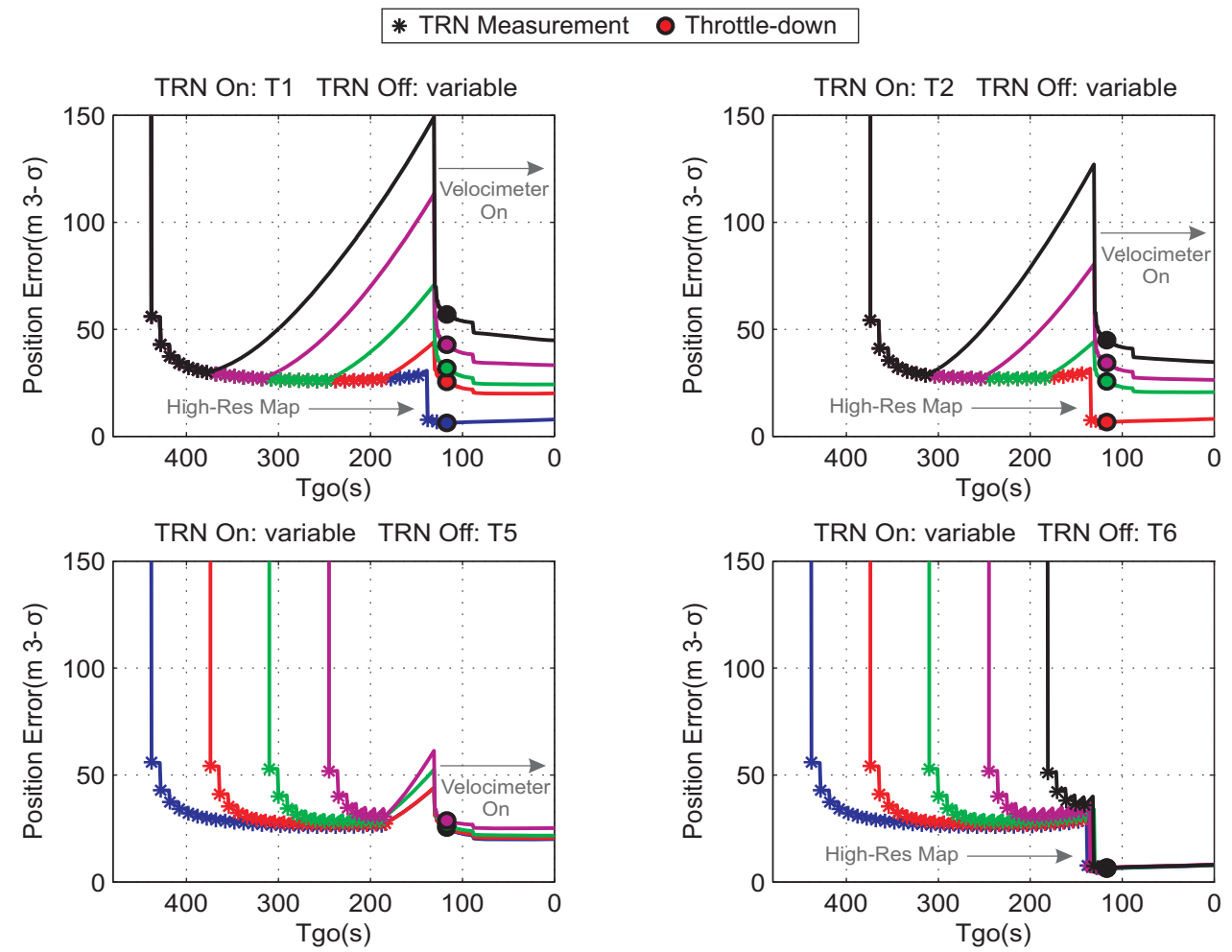

Fig. 5.2: Effect of varying the operating range of the TRN sensor on navigation performance Each line plots the navigation performance of a different operating range against the time to go until landing. Compare with Fig. 5.1. 


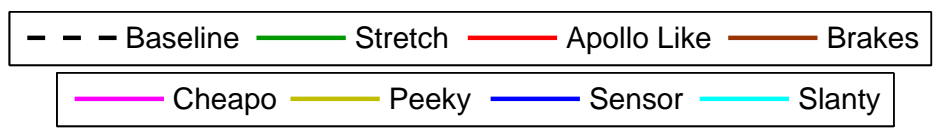

TRN Operating Altitude: T1 to T2

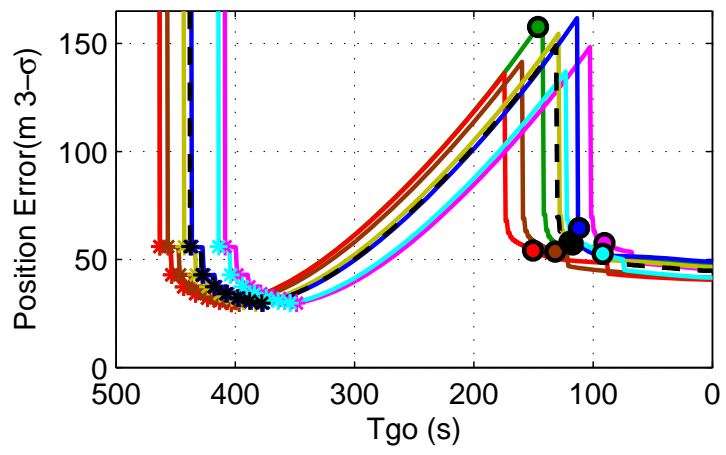

TRN Operating Altitude: T1 to T6

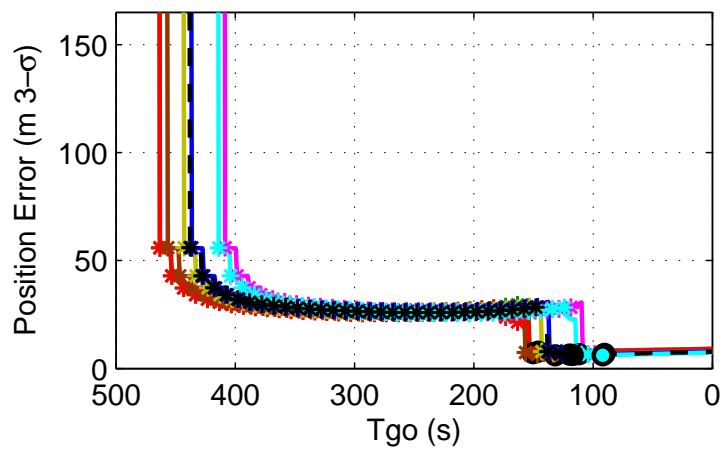

TRN Operating Altitude: T1 to T4

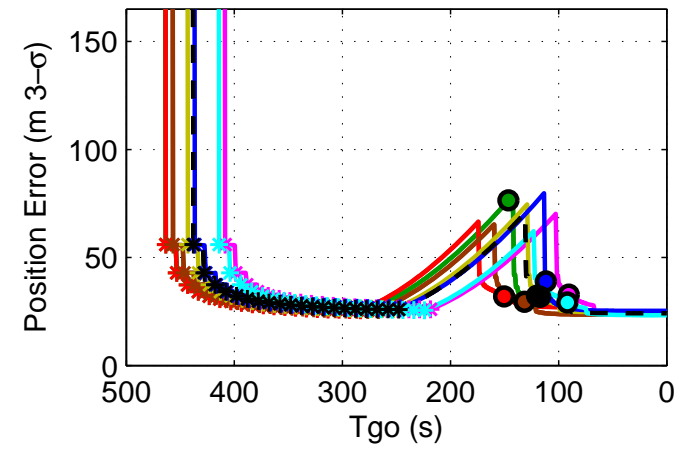

TRN Operating Altitude: T3 to T4

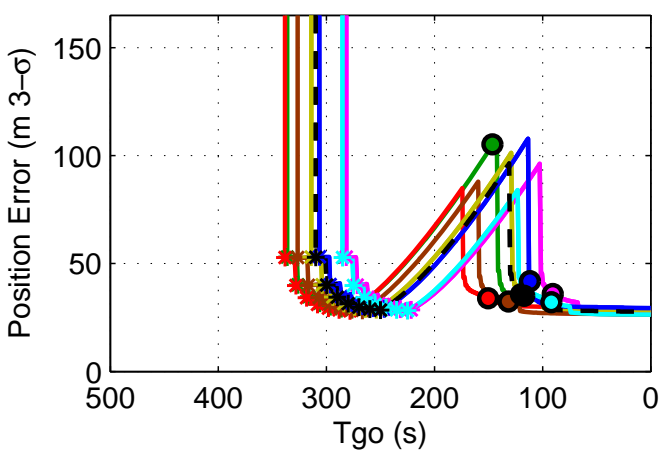

Fig. 5.3: Effect of varying the trajectory type on TRN performance.

\subsubsection{TRN Trajectory Study}

Next, it was desired to evaluate the sensitivity of the navigation results over the trajectory trade space. The navigation accuracy is shown in Fig. 5.3 for all eight nominal trajectories with four different TRN operating ranges. The navigation accuracy is plotted against $T_{g o}$, which is the time-to-go where landing is at 0 seconds. The majority of the difference between the results from each trajectory is caused by differences in the amount of time each trajectory requires from PDI to landing. Because of this, the lines that represent the results from each trajectory are simply time-shifted images of each other. This means that changing the trajectory type mainly affects how long each trajectory has to land after the TRN turns on at PDI and the velocimeter turns on at an altitude of $2 \mathrm{~km}$. Overall, the performance is not sensitive to the trajectory type. 

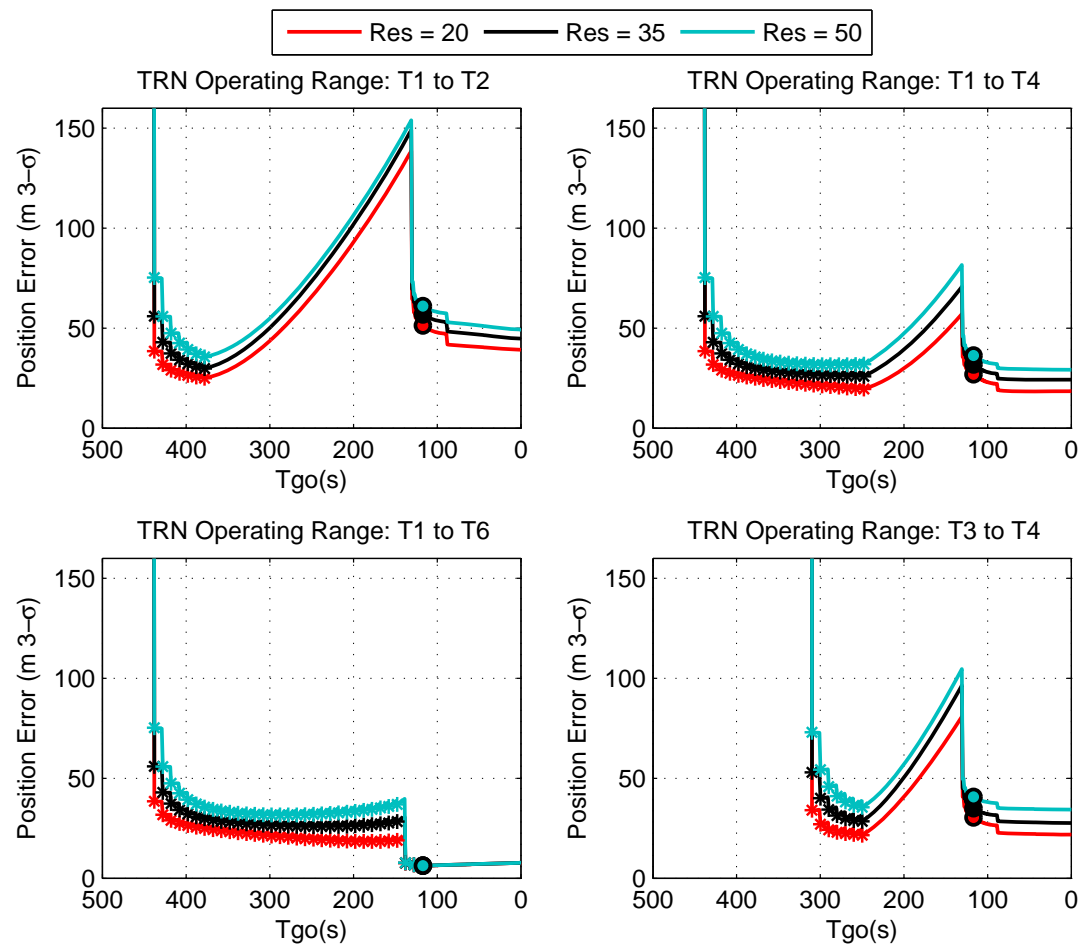

Fig. 5.4: Effect of varying the map resolution error for ranges greater than $5 \mathrm{~km}$ on TRN performance.

\subsubsection{Map Resolution}

The sensitivity of the navigation performance to the resolution of the onboard map $\left(\varepsilon_{\text {map }}^{s}\right)$ was also investigated. While the map resolution error for ranges within $5 \mathrm{~km}$ was held constant at $2 \mathrm{~m} \mathrm{3- \sigma}$, the map resolution for ranges beyond $5 \mathrm{~km}$ was varied from 20 to $50 \mathrm{~m} \mathrm{3-} \sigma$ (a change of $\pm 43 \%$ from the baseline value of $35 \mathrm{~m} \mathrm{3- \sigma}$ ). The results are shown in Fig. 5.4. While TRN was operating, the effect of the map resolution on navigation accuracy ranged from $20 \%$ to $30 \%$. The effect that it had at landing ranged from $0 \%$ to $25 \%$. This shows that the navigation performance is not very sensitive to the onboard map resolution over this range.

One observation that can be drawn from the plots in Fig. 5.4 is that the navigation accuracy is often even better than the map resolution after there have been multiple TRN measurements. This is because the velocity of the spacecraft relative to the surface is so high that the effect of the map resolution is not much more than high-frequency noise, 

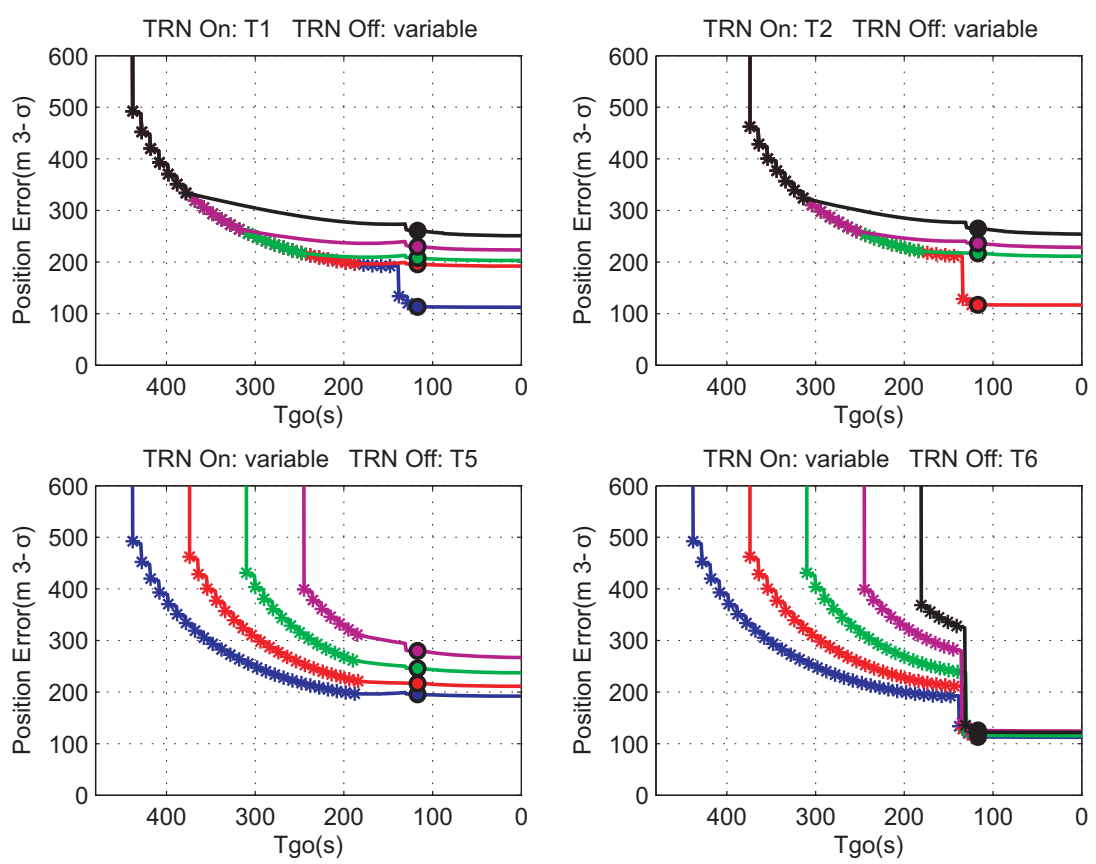

Fig. 5.5: Navigation errors for a landing at an outpost near the equator using an active TRN sensor with poor onboard map resolution. Compare with Fig. 5.2.

which the Kalman filter effectively filters out. The lower-left plot shows, however, that as the lander approaches the landing site and slows down, the frequency of the noise caused by the map resolution drops and becomes increasingly difficult to filter out.

A second map resolution study was performed to determine if an active TRN system using maps from the LRO mission would be able to support a landing near the equator. In this study, the trajectory remained the same, but the map resolution was changed to match the expected grid density at the equator, which would be $30 \mathrm{~m}$ in the north-south direction, $1200 \mathrm{~m}$ in the east-west direction, and $10 \mathrm{~m}$ in the vertical direction. It is also assumed that the terrain around the landing site has a ten-fold map resolution improvement. The predicted results of this situation over the possible operating ranges are shown in Fig. 5.5. It is apparent that the only operating ranges that can come close to the $100 \mathrm{~m} \mathrm{3-} \sigma$ precision landing requirement use the TRN sensor in the area with high-resolution maps. It is also apparent that the duration of time over which the TRN functions has a much greater effect when poorer resolution maps are used than it did with the baseline setup. 

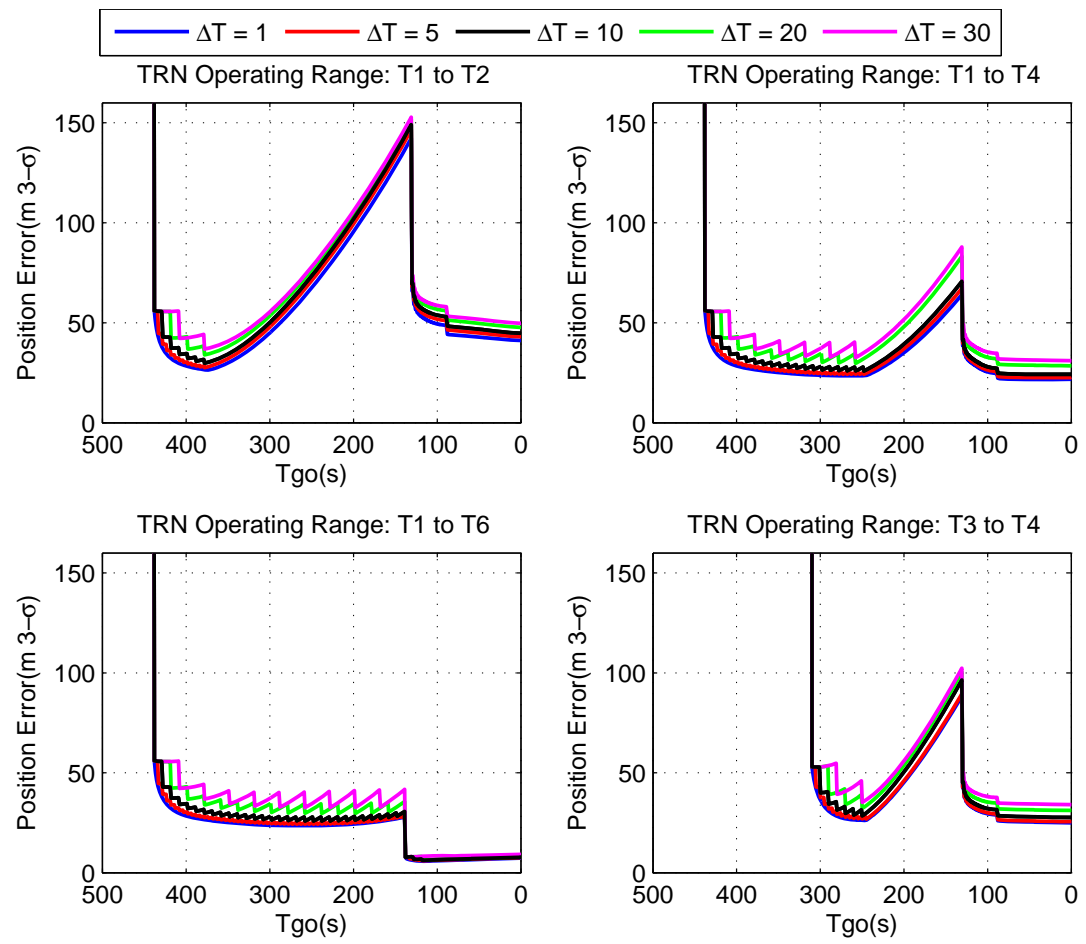

Fig. 5.6: Effect of varying the TRN measurement rate on TRN performance.

\subsubsection{Measurement Rate and Outages}

Another sensitivity study investigated the effects of the TRN measurement rate and possible measurement outages. The baseline design prescribes a TRN measurement to be taken and processed once every ten seconds. This study modified that rate to see what the effect would be if the actual TRN measurements were available more or less frequently, or if for any reason there were intermittent measurement outages. Fig. 5.6 shows the navigation results over a broad spectrum of measurement rates. The plot on the left particularly illustrates that TRN provides very robust measurements in that the results for $\Delta T=1$ are nearly the same as the results of $\Delta T=30$ after only 70 seconds. 
Table 5.1: LRS Orbital Elements and Initial Variance

\begin{tabular}{lcc}
\hline \hline Element & Value & Initial 3- $\boldsymbol{\sigma}$ Value \\
\hline Semi-major Axis & $6142 \mathrm{~km}$ & $0.2 \mathrm{~m}$ \\
Eccentricity & 0.57 & $0.1 \times 10^{-6}$ \\
Inclination & $44^{\circ}-58^{\circ}$ & $0.072 \operatorname{arcsec}$ \\
Argument of Perilune & $82^{\circ}-98^{\circ}$ & $0.180 \operatorname{arcsec}$ \\
Right Ascension & $0^{\circ}-360^{\circ}$ & $0.180 \operatorname{arcsec}$ \\
Mean Anomaly & $0^{\circ}-360^{\circ}$ & $0.00072 \operatorname{arcsec}$ \\
\hline
\end{tabular}

\subsection{Radiometric Navigation Studies}

The goal of the second phase of this research is to evaluate the performance of radiometric navigation using measurements during powered descent to one or two LRSs in lunar orbit or to a single LCT on the lunar surface. The LRSs are placed in a high-inclination frozen lunar orbit, which they share, separated by a mean anomaly of 180 degrees. The orbital elements for their orbit and the variances of these elements are shown in Table 5.1. When the orbital element variances are transformed to position and velocity variances, the errors are on the order of $10 \mathrm{~m} \mathrm{3- \sigma}$ and $2 \mathrm{~mm} / \mathrm{s} 3-\sigma$, respectively, with some variation as the mean anomaly changes.

There is some variability in the orientation of the LRS orbits due to three-body perturbations from the earth and the sun, solar pressure, and non-spherical gravity field perturbations from the moon. The inclination of the orbit and the argument of perilune both vary by $14^{\circ}$ and $16^{\circ}$, respectively. Preliminary analysis showed that the navigation performance had a limited sensitivity to these parameters, so they both were set at the middle of their range (i.e. the inclination is $51^{\circ}$ and the argument of perilune is $90^{\circ}$ ). The right ascension (RAAN) and the mean anomaly have the greatest effect on the navigation results and were sampled over the full $0^{\circ}-360^{\circ}$ range in all studies involving an LRS.

The surface beacons were analyzed by varying the position of a single LCT in the two grids illustrated in Fig. 5.7. A coarse $11 \times 11$ grid extends from the landing site up-range $350 \mathrm{~km}$ to the ground-track location directly under where PDI occurs with a spacing of 35 $\mathrm{km}$, and $80 \mathrm{~km}$ off-track (as far as the lander can see from its altitude at PDI, subject to the elevation constraint for visibility) with a spacing of $8 \mathrm{~km}$. LCTs that have no contact 


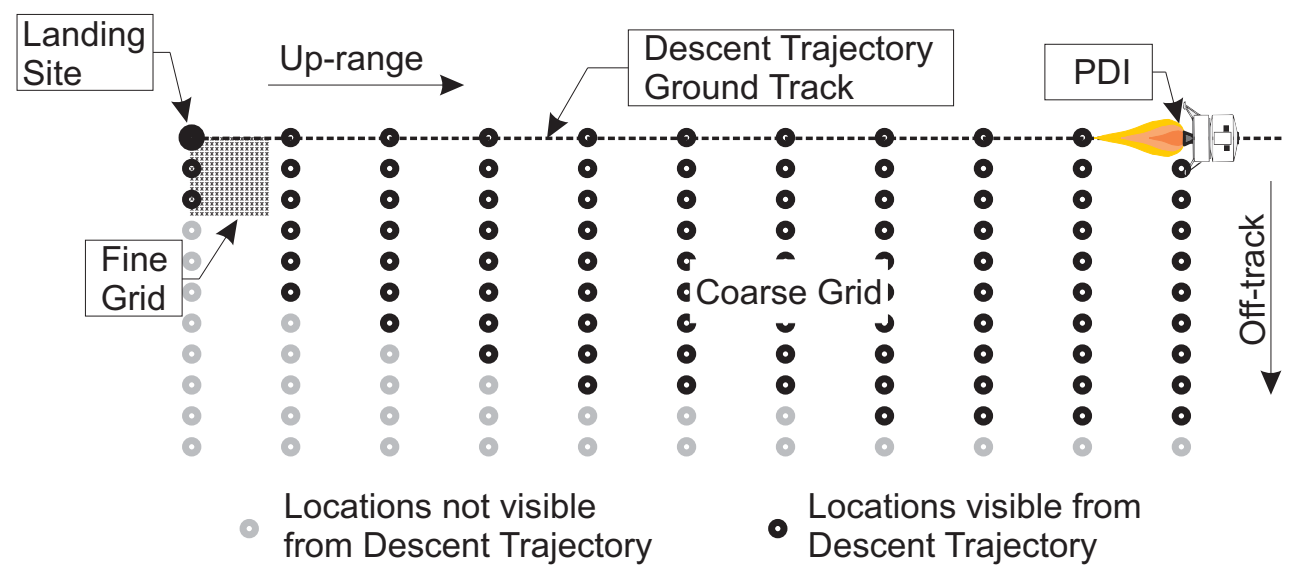

Fig. 5.7: Grids for LCT placement.

with the lander during the final descent are ignored. The fine grid focuses more on LCTs located near the landing site. This grid extends $20 \mathrm{~km}$ up-range and $15 \mathrm{~km}$ off-track with $1 \mathrm{~km}$ spacing in both directions.

\subsubsection{Single Orbiting Beacon Study}

An example of the time history of the lander's relative navigation performance using a single orbiting beacon is shown in Fig. 5.8. As mentioned previously, radiometric measurements are assumed to be available during the two orbits (4 hours) prior to the deorbit burn. The sawtooth appearance of the plot is caused by the LRS coming in and out of sight as the lander orbits with a period of approximately 2 hours. The LRS comes into view one last time during the final descent. With the aid of the baseline sensor suite (IMU, star camera, altimeter, and velocimeter), the radiometric navigation performance is shown to be approximately $200 \mathrm{~m} \mathrm{3-} \sigma$ when the right ascension of the LRS is rotated $180^{\circ}$ relative to the right ascension of the lander's orbit and the LRS is at apolune when the deorbit burn occurs.

To investigate all possible arrival conditions, the LRS mean anomalies and right ascensions were sampled by selecting 16 evenly-spaced values for the right ascension and 10 evenly-spaced values for the mean anomaly (including the case considered above). The navigation performances at PDI for all 160 possible cases are shown in Fig. 5.9. The values in 
Relative LRS Right Ascension: $-180^{\circ}$ LRS Mean Anomaly: $180^{\circ}$
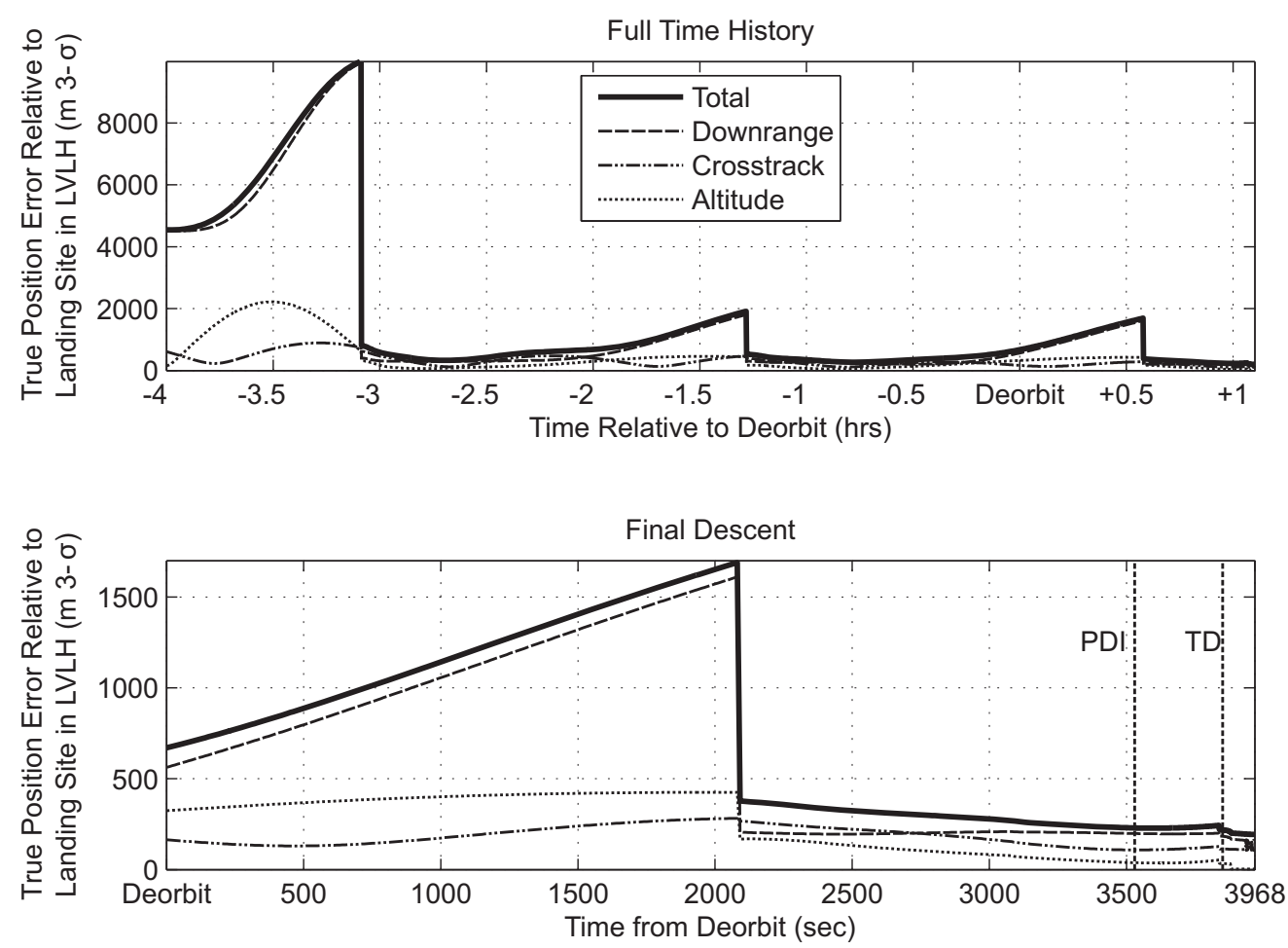

Fig. 5.8: Time history of the navigation error of the lander relative to the landing site using radiometric measurements to a single LRS for two orbits prior to the final descent and during the descent.

these figures are color-coded with the best results highlighted in blue and the worst results highlighted in red. It was observed that without exception, the navigation errors decreased uniformly throughout the trajectory so that the best cases at PDI gave the best results all the way to landing. The statistics of the $3-\sigma$ inertial and relative navigation accuracies from all of the mean anomaly and right ascension combinations at all four key locations in the trajectory are shown in Table 5.2.

The two key factors that drove performance were the duration of radiometric tracking and how much the geometry changed over time. Intuitively, the cases with longer durations of tracking performed best and cases where no tracking was available during the final descent (i.e., most of the geometries in the two far-right columns) performed quite poorly. The geometries in which the LRS was at perilune when the deorbit burn occurred (far left column) provide visibility during the deorbit burn and the first portion of the descent. 


\begin{tabular}{|c|c|c|c|c|c|c|c|c|c|c|c|}
\hline \multirow{2}{*}{\multicolumn{2}{|c|}{ PDI }} & \multicolumn{10}{|c|}{ LRS Mean Anomaly at Deorbit (deg) } \\
\hline & & 0 & 36 & 72 & 108 & 144 & 180 & 216 & 252 & 288 & 324 \\
\hline \multirow{16}{*}{ 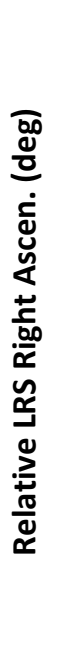 } & -180 & 472 & 192 & 207 & 245 & 223 & 229 & 219 & 212 & 210 & 208 \\
\hline & -157.5 & 464 & 217 & 211 & 211 & 207 & 204 & 206 & 210 & 208 & 250 \\
\hline & -135 & 468 & 214 & 210 & 194 & 187 & 198 & 206 & 217 & 239 & 309 \\
\hline & -112.5 & 482 & 213 & 193 & 182 & 185 & 205 & 224 & 248 & 287 & 1964 \\
\hline & -90 & 511 & 250 & 183 & 194 & 198 & 227 & 246 & 298 & 364 & 2036 \\
\hline & -67.5 & 545 & 205 & 177 & 215 & 219 & 236 & 278 & 325 & 1796 & 1903 \\
\hline & -45 & 424 & 197 & 233 & 220 & 219 & 254 & 272 & 283 & 1485 & 921 \\
\hline & -22.5 & 174 & 283 & 235 & 216 & 236 & 239 & 249 & 243 & 1115 & 726 \\
\hline & 0 & 178 & 290 & 223 & 216 & 222 & 228 & 233 & 237 & 930 & 688 \\
\hline & 22.5 & 181 & 268 & 223 & 220 & 215 & 222 & 245 & 269 & 872 & 731 \\
\hline & 45 & 259 & 328 & 237 & 216 & 211 & 250 & 306 & 319 & 890 & 846 \\
\hline & 67.5 & 516 & 406 & 217 & 225 & 242 & 352 & 342 & 330 & 990 & 1250 \\
\hline & 90 & 512 & 269 & 243 & 220 & 301 & 334 & 310 & 391 & 1302 & 1651 \\
\hline & 112.5 & 484 & 206 & 249 & 216 & 290 & 279 & 395 & 487 & 1515 & 200 \\
\hline & 135 & 495 & 202 & 203 & 218 & 221 & 412 & 368 & 301 & 233 & 195 \\
\hline & 157.5 & 484 & 194 & 201 & 213 & 228 & 285 & 260 & 234 & 211 & 208 \\
\hline
\end{tabular}

Fig. 5.9: Relative navigation results at PDI in meters 3- $\sigma$ using radiometric measurements to a single LRS.

Table 5.2: Statistics of the navigation performance metrics over the mean anomaly and right ascension trade space for the single orbiting beacon case. Compare with Fig. 5.9.

\begin{tabular}{ccccc}
\hline \hline Performance Metric & Median & Min & Max & Average \\
\hline \multicolumn{4}{c}{ Inertial Navigation Performance } \\
PDI & $191 \mathrm{~m}$ & $101 \mathrm{~m}$ & $2031 \mathrm{~m}$ & $339 \mathrm{~m}$ \\
Throttle-down & $187 \mathrm{~m}$ & $103 \mathrm{~m}$ & $626 \mathrm{~m}$ & $240 \mathrm{~m}$ \\
HDA & $178 \mathrm{~m}$ & $101 \mathrm{~m}$ & $595 \mathrm{~m}$ & $231 \mathrm{~m}$ \\
Landing & $158 \mathrm{~m}$ & $95 \mathrm{~m}$ & $510 \mathrm{~m}$ & $202 \mathrm{~m}$ \\
\hline Relative Navigation Performance & \\
PDI & $238 \mathrm{~m}$ & $174 \mathrm{~m}$ & $2036 \mathrm{~m}$ & $381 \mathrm{~m}$ \\
Throttle-down & $235 \mathrm{~m}$ & $175 \mathrm{~m}$ & $642 \mathrm{~m}$ & $284 \mathrm{~m}$ \\
HDA & $228 \mathrm{~m}$ & $174 \mathrm{~m}$ & $611 \mathrm{~m}$ & $276 \mathrm{~m}$ \\
Landing & $212 \mathrm{~m}$ & $170 \mathrm{~m}$ & $529 \mathrm{~m}$ & $252 \mathrm{~m}$ \\
\hline
\end{tabular}


Because the LRS was at its lowest altitude and moving at its highest velocity, the rangerate measurements were most effective in these cases. However, because tracking occurred at the beginning of the descent, these cases performed quite poorly during the final portion of the descent with a few exceptions where the LRS followed the lander throughout a significant portion of its trajectory and observed it from multiple angles. These two key factors are discussed in more detail in Appendix B.

Because the mean anomaly is much easier to modify and control than the right ascension, the goal is to identify a mean anomaly for each possible right ascension that would meet the requirement for the four performance metrics. It was observed that this was possible at PDI and, in most cases, at throttle-down, but not possible at HDA and landing. For each right ascension, a mean anomaly can be selected to achieve a relative navigation performance at PDI and throttle-down near $200 \mathrm{~m} \mathrm{3- \sigma}$. However, the requirements to support HDA and landing were not met as the inertial navigation accuracies (which do not include map-tie) never fall below $100 \mathrm{~m} \mathrm{3- \sigma .}$

Finally, the sensitivity to multiple parameters including the accuracy of the radiometric measurements, the elevation-angle constraint for LRS visibility, and the initial covariances of the LRS, lander, and landing site (map-tie) was determined. In addition, the effect of using TRN measurements just before throttle-down (from T5 to T6) and only using radiometric measurements from deorbit to landing was also evaluated. The results for this sensitivity study are shown in Fig. 5.10.

Variations in range and range-rate measurement accuracy and the initial covariance of the lander and LRS did not have a large affect on the navigation performance. The map-tie error did have a significant effect, but even if the map-tie error was eliminated completely, the $100 \mathrm{~m}$ requirement at HDA cannot be met. The elevation constraint for visibility plays a significant role in a few trajectories, but does not affect performance in most of the trajectories that otherwise have good navigation performance. When the TRN sensor is turned on, it is clear that nearly all of the error at and after throttle-down is eliminated, which would mean that radiometric navigation could support improved accuracy at PDI until the more 


\begin{tabular}{|c|c|c|c|c|c|c|c|c|}
\hline \multirow{3}{*}{ Parameter } & \multirow{3}{*}{$\begin{array}{c}\text { Baseline } \\
(3-\sigma)\end{array}$} & \multirow{3}{*}{$\begin{array}{l}\text { Value } \\
(3-\sigma)\end{array}$} & \multicolumn{6}{|c|}{ Effect on Relative Position Error of Lander } \\
\hline & & & \multicolumn{2}{|c|}{ PDI } & \multicolumn{2}{|c|}{ Throttle-Down } & \multicolumn{2}{|c|}{ Landing } \\
\hline & & & Med. \% & Max. \% & Med. \% & Max. \% & Med. \% & Max. \% \\
\hline \multirow{2}{*}{ Range Bias/Noise } & \multirow{2}{*}{$35 \mathrm{~ns}$} & $10 \mathrm{~ns}$ & $-1.2 \%$ & $-4.3 \%$ & $-1.2 \%$ & $-4.0 \%$ & $-1.2 \%$ & $-3.5 \%$ \\
\hline & & $50 \mathrm{~ns}$ & $0.7 \%$ & $2.9 \%$ & $0.7 \%$ & $2.8 \%$ & $0.7 \%$ & $2.9 \%$ \\
\hline \multirow{2}{*}{ Doppler Bias/Noise } & \multirow{2}{*}{$0.5 \mathrm{~mm} / \mathrm{s}$} & $0.05 \mathrm{~mm} / \mathrm{s}$ & $0.0 \%$ & $-0.3 \%$ & $0.0 \%$ & $-0.3 \%$ & $-0.1 \%$ & $-0.3 \%$ \\
\hline & & $5 \mathrm{~mm} / \mathrm{s}$ & $0.5 \%$ & $2.8 \%$ & $0.4 \%$ & $1.6 \%$ & $0.7 \%$ & $2.5 \%$ \\
\hline \multirow{2}{*}{$\begin{array}{l}\text { Elevation Constraint for } \\
\text { Visibility }\end{array}$} & \multirow{2}{*}{$10 \mathrm{deg}$} & $1 \mathrm{deg}$ & $-1.0 \%$ & $-83.2 \%$ & $-0.8 \%$ & $-55.9 \%$ & $-0.6 \%$ & $-49.6 \%$ \\
\hline & & 20 deg & $1.3 \%$ & $1025.6 \%$ & $0.8 \%$ & $202.2 \%$ & $0.7 \%$ & $150.3 \%$ \\
\hline \multirow{2}{*}{ Initial LRS Cov } & \multirow{2}{*}{$\sim 10 \mathrm{~m}$} & $\sim 1 \mathrm{~m}$ & $0.0 \%$ & $0.0 \%$ & $0.0 \%$ & $0.0 \%$ & $0.0 \%$ & $0.0 \%$ \\
\hline & & $\sim 30 \mathrm{~m}$ & $0.0 \%$ & $0.0 \%$ & $0.0 \%$ & $0.0 \%$ & $0.0 \%$ & $0.0 \%$ \\
\hline Initial Lander Cov & $1500 \mathrm{~m}$ & $110 \mathrm{~m}$ & $-2.3 \%$ & $-27.2 \%$ & $-1.9 \%$ & $-18.0 \%$ & $-1.4 \%$ & $-12.5 \%$ \\
\hline Map Tie & $100 \mathrm{~m}$ & $30 \mathrm{~m}$ & $-17.7 \%$ & $-36.9 \%$ & $-18.3 \%$ & $-36.4 \%$ & $-22.7 \%$ & $-38.8 \%$ \\
\hline Late TRN & Off & On & $0.0 \%$ & $0.0 \%$ & $-97.2 \%$ & $-99.0 \%$ & $-96.5 \%$ & $-98.5 \%$ \\
\hline Tracking Before Deorbit & On & Off & $45.7 \%$ & $494.5 \%$ & $20.9 \%$ & $91.1 \%$ & $13.7 \%$ & $44.8 \%$ \\
\hline \multicolumn{3}{|c|}{ Median Position Error with Baseline Setup: } & \multicolumn{2}{|c|}{$238 \mathrm{~m}$} & \multicolumn{2}{|c|}{$235 \mathrm{~m}$} & \multicolumn{2}{|c|}{$212 \mathrm{~m}$} \\
\hline & & \multicolumn{3}{|c|}{ Legend: } & & & & \\
\hline & & \multicolumn{3}{|c|}{ No significant Change } & & & & \\
\hline & & \multirow{2}{*}{\multicolumn{3}{|c|}{ Significant Improvement }} & & & & \\
\hline & & & & & & & & \\
\hline
\end{tabular}

Fig. 5.10: Sensitivity of relative navigation results to key parameters for the single orbiting beacon study.

robust TRN measurements are available. Finally, using radiometric measurements only during the final descent causes significantly worse performance, particularly for trajectories that do not support tracking during the final descent.

\subsubsection{Two Coplanar Orbiting Beacons Study}

The effect of adding radiometric measurements to a second LRS was evaluated in the next stage of the analysis. The second LRS was placed in the same orbit with the same element variances and separated from the first by a mean anomaly of 180 degrees. Because of the symmetry in the mean anomaly, this reduced the possible right ascension and mean anomaly combinations by a factor of two. The relative navigation results at PDI are shown in Fig. 5.11 and the statistics of all the performance metrics over all possible mean anomaly and right ascension combinations are displayed in Table 5.3.

It can be seen that the effect of the right ascension and mean anomaly of the LRS orbit was reduced when compared to the single orbiting beacon study. The results are generally better than the single beacon study because of an increase in the amount of time 


\begin{tabular}{|c|c|c|c|c|c|c|}
\hline \multirow{2}{*}{\multicolumn{2}{|c|}{ PDI }} & \multicolumn{5}{|c|}{ Mean anomaly of LRS 1/LRS 2 at De-Orbit (deg) } \\
\hline & & \multirow{2}{*}{$\frac{\mathbf{0} / \mathbf{1 8 0}}{182}$} & \multirow{2}{*}{$\begin{array}{c}\mathbf{3 6 / 2 1 6} \\
150\end{array}$} & \multirow{2}{*}{$\begin{array}{c}\mathbf{7 2 / 2 5 2} \\
150\end{array}$} & \multirow{2}{*}{$\begin{array}{c}108 / 288 \\
149\end{array}$} & \multirow{2}{*}{$\begin{array}{c}\mathbf{1 4 4 / 3 2 4} \\
157\end{array}$} \\
\hline \multirow{16}{*}{ 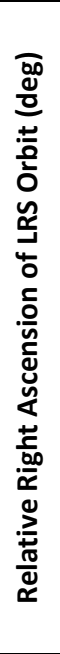 } & -180 & & & & & \\
\hline & -157.5 & 172 & 151 & 150 & 149 & 156 \\
\hline & -135 & 164 & 151 & 150 & 149 & 161 \\
\hline & -112.5 & 167 & 151 & 150 & 149 & 169 \\
\hline & -90 & 168 & 151 & 150 & 150 & 182 \\
\hline & -67.5 & 165 & 150 & 150 & 183 & 176 \\
\hline & -45 & 168 & 150 & 151 & 174 & 172 \\
\hline & -22.5 & 148 & 150 & 151 & 176 & 182 \\
\hline & 0 & 148 & 150 & 151 & 192 & 197 \\
\hline & 22.5 & 149 & 150 & 151 & 201 & 191 \\
\hline & 45 & 151 & 150 & 151 & 192 & 181 \\
\hline & 67.5 & 153 & 150 & 150 & 180 & 172 \\
\hline & 90 & 160 & 150 & 150 & 172 & 173 \\
\hline & 112.5 & 165 & 150 & 150 & 173 & 157 \\
\hline & 135 & 173 & 150 & 149 & 149 & 159 \\
\hline & 157.5 & 182 & 150 & 149 & 149 & 161 \\
\hline
\end{tabular}

Fig. 5.11: Relative navigation results at PDI in meters $3-\boldsymbol{\sigma}$ using radiometric measurements to two LRSs.

Table 5.3: Statistics of the navigation performance metrics over the mean anomaly and right ascension trade space for the two coplanar orbiting beacon case. Compare to Fig. 5.11.

\begin{tabular}{|c|c|c|c|c|}
\hline Performance Metric & Median & Min & Max & Average \\
\hline \multicolumn{5}{|c|}{ Inertial Navigation Performance } \\
\hline PDI & $52 \mathrm{~m}$ & $42 \mathrm{~m}$ & $143 \mathrm{~m}$ & $72 \mathrm{~m}$ \\
\hline Throttle-down & $50 \mathrm{~m}$ & $45 \mathrm{~m}$ & $136 \mathrm{~m}$ & $71 \mathrm{~m}$ \\
\hline HDA & $49 \mathrm{~m}$ & $44 \mathrm{~m}$ & $133 \mathrm{~m}$ & $70 \mathrm{~m}$ \\
\hline Landing & $39 \mathrm{~m}$ & $30 \mathrm{~m}$ & $117 \mathrm{~m}$ & $59 \mathrm{~m}$ \\
\hline \multicolumn{5}{|c|}{ Relative Navigation Performance } \\
\hline PDI & $151 \mathrm{~m}$ & $148 \mathrm{~m}$ & $201 \mathrm{~m}$ & $161 \mathrm{~m}$ \\
\hline Throttle-down & $150 \mathrm{~m}$ & $149 \mathrm{~m}$ & $196 \mathrm{~m}$ & $161 \mathrm{~m}$ \\
\hline HDA & $150 \mathrm{~m}$ & $148 \mathrm{~m}$ & $194 \mathrm{~m}$ & $160 \mathrm{~m}$ \\
\hline Landing & $146 \mathrm{~m}$ & $144 \mathrm{~m}$ & $183 \mathrm{~m}$ & $155 \mathrm{~m}$ \\
\hline
\end{tabular}


when LRSs are visible to the lander and an increase in the variation of the measurement geometries. In the two beacon study, the mean anomaly of the constellation can easily be selected for each ascending node to ensure relative navigation errors of $150 \mathrm{~m} \mathrm{3-} \sigma$ or less at PDI, throttle-down, HDA, and landing. However, this still does not meet the requirement for a navigation accuracy of $100 \mathrm{~m} \mathrm{3- \sigma}$ at HDA and landing.

It is important to note here that in cases without TRN or other surface aids, the inertial navigation covariance of the lander is independent of the covariance of the landing site position error. Because of this, the relative navigation performance can be calculated by the root-sum-square of the inertial navigation performance and the covariance of the landing site position error. This is important because in most cases, inertial navigation performances are much better than $100 \mathrm{~m} \mathrm{3- \sigma}$. If the uncertainty in the landing site position can be reduced to $60 \mathrm{~m} \mathrm{3-} \sigma$ in the north and east directions, then radiometric navigation relative to two orbiting beacons could support all phases of the descent with a relative navigation error of $100 \mathrm{~m} \mathrm{3- \sigma}$.

The same parameter study that was performed for the single orbiting beacon case was performed for the two orbiting beacon case and yielded very similar results, which are summarized in Fig. 5.12. Radiometric navigation performance was again insensitive to variations in measurement accuracy and the initial covariance of the lander and the LRSs. In this study, the performance was insensitive to the elevation constraint for visibility in all cases. The effect of eliminating tracking before the deorbit burn was not as dramatic as it was in the single orbiting beacon study, but remained significant.

\subsubsection{Single Surface Beacon Study}

The final stage of this analysis focused on the benefits of taking range and range-rate measurements to a single LCT surface beacon. The position error of the LCT with respect to the landing site was assumed negligible in this analysis, but can be added to the results (root-sum-square) as an independent error. LCTs in both the fine and coarse grids are considered in this study. Those in the coarse grid are assumed to have enough power to communicate with the lander while it is in a circular orbit at an altitude of $100 \mathrm{~km}$ during 


\begin{tabular}{|c|c|c|c|c|c|}
\hline \multirow[b]{2}{*}{ Parameter } & \multirow[b]{2}{*}{$\begin{array}{c}\text { Baseline } \\
(3-\sigma)\end{array}$} & \multirow[b]{2}{*}{$\begin{array}{l}\text { Value } \\
(3-\sigma)\end{array}$} & \multicolumn{3}{|c|}{ Average Effect on Relative Position Error of Lander } \\
\hline & & & PDI & Throttle-Down & Landing \\
\hline \multirow{2}{*}{ Range Bias/Noise } & \multirow{2}{*}{$35 \mathrm{~ns}$} & $10 \mathrm{~ns}$ & $-2.3 \mathrm{~m} \quad(-1.4 \%)$ & $-2.3 \mathrm{~m} \quad(-1.4 \%)$ & $-2.5 \mathrm{~m} \quad(-1.6 \%)$ \\
\hline & & $50 \mathrm{~ns}$ & $+1.1 \mathrm{~m} \quad(+0.7 \%)$ & $+1.1 \mathrm{~m} \quad(+0.7 \%)$ & $+1.2 \mathrm{~m} \quad(+0.8 \%)$ \\
\hline \multirow{2}{*}{ Doppler Bias/Noise } & \multirow{2}{*}{$0.5 \mathrm{~mm} / \mathrm{s}$} & $0.05 \mathrm{~mm} / \mathrm{s}$ & $-0.1 \mathrm{~m} \quad(-0.1 \%)$ & $-0.1 \mathrm{~m} \quad(-0.1 \%)$ & $-0.1 \mathrm{~m} \quad(-0.1 \%)$ \\
\hline & & $5 \mathrm{~mm} / \mathrm{s}$ & $+0.9 \mathrm{~m} \quad(+0.6 \%)$ & $+0.8 \mathrm{~m} \quad(+0.5 \%)$ & $+0.7 \mathrm{~m} \quad(+0.5 \%)$ \\
\hline \multirow{2}{*}{$\begin{array}{l}\text { Elevation Constraint for } \\
\text { Visibility }\end{array}$} & \multirow{2}{*}{10 deg } & $1 \mathrm{deg}$ & $-2.1 \mathrm{~m} \quad(-1.2 \%)$ & $-2.4 \mathrm{~m} \quad(-1.4 \%)$ & $-2.2 \mathrm{~m} \quad(-1.3 \%)$ \\
\hline & & $20 \mathrm{deg}$ & $+4.9 \mathrm{~m} \quad(+3.2 \%)$ & $+2.0 \mathrm{~m} \quad(+1.2 \%)$ & $+1.8 \mathrm{~m} \quad(+1.1 \%)$ \\
\hline \multirow{2}{*}{ Initial LRS Cov } & \multirow{2}{*}{$\sim 10 \mathrm{~m}$} & $\sim 1 \mathrm{~m}$ & $0.0 \mathrm{~m} \quad 0.00 \%$ & $0.0 \mathrm{~m} \quad 0.00 \%$ & $0.0 \mathrm{~m} \quad 0.00 \%$ \\
\hline & & $\sim 30 \mathrm{~m}$ & $+0.1 \mathrm{~m} \quad(+0.0 \%)$ & $+0.1 \mathrm{~m} \quad(+0.0 \%)$ & $+0.1 \mathrm{~m} \quad(+0.1 \%)$ \\
\hline Initial Lander Cov & $1500 \mathrm{~m}$ & $110 \mathrm{~m}$ & $0.0 \mathrm{~m} \quad 0.00 \%$ & $0.0 \mathrm{~m} \quad 0.00 \%$ & $0.0 \mathrm{~m} \quad 0.00 \%$ \\
\hline Map Tie & $100 \mathrm{~m}$ & $30 \mathrm{~m}$ & $-76.3 m \quad(-48.4 \%)$ & $-76.4 m \quad(-48.4 \%)$ & $-82.1 m(-53.9 \%)$ \\
\hline Tracking Before Deorbit & On & Off & $+59.5 \mathrm{~m}(+32.7 \%)$ & $+32.0 m(+17.8 \%)$ & $+20.4 m(+12.0 \%)$ \\
\hline \multicolumn{3}{|c|}{ Average Position Error with Baseline Setup: } & $160.71 \mathrm{~m}$ & $160.68 \mathrm{~m}$ & $155.08 \mathrm{~m}$ \\
\hline & & \multicolumn{2}{|c|}{ Legend: } & & \\
\hline & & \multicolumn{2}{|c|}{ No significant Change } & & \\
\hline & & \multicolumn{2}{|c|}{ Significant Improvement } & & \\
\hline & & \multicolumn{2}{|c|}{ Significant Degradation } & & \\
\hline
\end{tabular}

Fig. 5.12: Sensitivity of relative navigation results to operational parameters for the two coplanar orbiting beacon study.

the two orbits prior to the deorbit burn, subject to the elevation angle constraint. The LCTs in the fine grid are allowed to communicate with the lander only during the final descent, also subject to the elevation angle constraint.

The navigation performances for the baseline case are shown in Fig. 5.13 for the coarse grid at PDI, throttle-down and landing. There is a definite line in the first chart that shows which LCTs are visible to the lander at or before PDI. When the lander attempts to navigate relative to LCTs located closer to the landing site, it has high navigation errors at PDI because it has had no direct contact with the LCT since the previous orbit. LCTs farther up-range, however, can support PDI very well. The results at throttle-down and landing show that any LCT location that is not on the ground-track of the final descent trajectory is able to support a precision landing with relative navigation accuracies below $100 \mathrm{~m} \mathrm{3-} \sigma$. LCTs on the ground-track, however, are unable to observe the cross-track position of the lander because the position information from both the range and range-rate measurements was in the plane of the orbit. The factors that affect the performance of the surface-beacon-relative navigation results are discussed in more detail in Appendix B. 


\begin{tabular}{|c|c|c|c|c|c|c|c|c|c|c|c|c|}
\hline \multirow{2}{*}{\multicolumn{2}{|c|}{ PDI }} & \multicolumn{11}{|c|}{ Off-Track Location of Beacon (km) } \\
\hline & & \multirow[t]{2}{*}{80} & \multirow[t]{2}{*}{72} & \multirow[t]{2}{*}{64} & \multirow[t]{2}{*}{56} & \multirow[t]{2}{*}{48} & \multirow[t]{2}{*}{40} & \multirow[t]{2}{*}{32} & \multirow[t]{2}{*}{24} & \multirow{2}{*}{$\begin{array}{c}16 \\
1525\end{array}$} & \multirow{2}{*}{$\begin{array}{c}8 \\
1495\end{array}$} & \multirow{2}{*}{$\frac{\mathbf{0}}{1436}$} \\
\hline$\widehat{\boldsymbol{E}}$ & 0 & & & & & & & & & & & \\
\hline 픔 & 35 & & & & & & 1651 & 1611 & 1572 & 1541 & 1522 & 1431 \\
\hline ¿̊ & 70 & & & & & 1696 & 1659 & 1620 & 1585 & 1559 & 1554 & 1418 \\
\hline ఖ్ల & 105 & & & & 1735 & 1701 & 1664 & 1627 & 1595 & 1577 & 1586 & 1405 \\
\hline 4 & 140 & & & 1773 & 1743 & 1709 & 1672 & 1637 & 1608 & 1599 & 1620 & 1402 \\
\hline 드 & 175 & & & 1780 & 1750 & 1716 & 1681 & 1647 & 1623 & 1625 & 1641 & 1403 \\
\hline 莺 & 210 & & & 1787 & 1756 & 1723 & 1689 & 1659 & 1641 & 1653 & 1624 & 1408 \\
\hline 9 & 245 & & 1821 & 1793 & 1762 & 63 & 64 & 66 & 71 & 81 & 122 & 304 \\
\hline ำ & 280 & & 1826 & 62 & 62 & 52 & 53 & 55 & 51 & 60 & 92 & 276 \\
\hline$\underset{\pi}{\pi}$ & 315 & & 51 & 50 & 49 & 47 & 47 & 48 & 50 & 57 & 85 & 256 \\
\hline ㅇํㅁ & 350 & & 52 & 50 & 44 & 44 & 43 & 44 & 46 & 52 & 78 & 238 \\
\hline
\end{tabular}

\begin{tabular}{|c|c|c|c|c|c|c|c|c|c|c|c|c|}
\hline \multirow{2}{*}{\multicolumn{2}{|c|}{$\begin{array}{c}\text { Throttle- } \\
\text { Down }\end{array}$}} & \multicolumn{11}{|c|}{ Off-Track Location of Beacon (km) } \\
\hline & & \multirow[t]{2}{*}{80} & \multirow[t]{2}{*}{72} & \multirow[t]{2}{*}{64} & \multirow[t]{2}{*}{56} & \multirow[t]{2}{*}{48} & \multirow[t]{2}{*}{40} & \multirow[t]{2}{*}{32} & \multirow[t]{2}{*}{24} & \multirow{2}{*}{$\frac{16}{37}$} & \multirow{2}{*}{$\frac{8}{19}$} & \multirow{2}{*}{$\frac{\mathbf{0}}{712}$} \\
\hline$\overline{\boldsymbol{\varepsilon}}$ & 0 & & & & & & & & & & & \\
\hline 总 & 35 & & & & & & 41 & 38 & 37 & 39 & 46 & 751 \\
\hline ธุ & 70 & & & & & 42 & 39 & 39 & 40 & 42 & 55 & 645 \\
\hline$\stackrel{\mathbb{D}}{0}$ & 105 & & & & 46 & 42 & 41 & 41 & 42 & 46 & 61 & 537 \\
\hline$\overline{0}$ & 140 & & & 50 & 46 & 44 & 44 & 44 & 45 & 49 & 65 & 454 \\
\hline ఏ & 175 & & & 52 & 48 & 47 & 47 & 47 & 48 & 52 & 68 & 393 \\
\hline 莺 & 210 & & & 53 & 51 & 50 & 50 & 51 & 52 & 55 & 71 & 348 \\
\hline בَ & 245 & & 69 & 57 & 55 & 54 & 54 & 54 & 55 & 58 & 72 & 313 \\
\hline 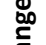 & 280 & & 64 & 60 & 60 & 59 & 59 & 59 & 59 & 62 & 76 & 286 \\
\hline$\underset{\mathbb{1}}{\mathbb{1}}$ & 315 & & 68 & 65 & 65 & 63 & 63 & 63 & 64 & 66 & 79 & 265 \\
\hline ํํㅁ & 350 & & 73 & 72 & 70 & 69 & 69 & 70 & 71 & 71 & 83 & 248 \\
\hline
\end{tabular}

\begin{tabular}{|c|c|c|c|c|c|c|c|c|c|c|c|c|}
\hline \multirow{2}{*}{\multicolumn{2}{|c|}{ Landing }} & \multicolumn{11}{|c|}{ Off-Track Location of Beacon (km) } \\
\hline & & \multirow[t]{2}{*}{80} & \multirow[t]{2}{*}{72} & \multirow[t]{2}{*}{64} & \multirow[t]{2}{*}{56} & \multirow[t]{2}{*}{48} & \multirow[t]{2}{*}{40} & \multirow[t]{2}{*}{32} & \multirow[t]{2}{*}{24} & \multirow{2}{*}{$\frac{16}{8}$} & \multirow{2}{*}{$\frac{8}{6}$} & \multirow{2}{*}{$\frac{\mathbf{0}}{70}$} \\
\hline $\bar{\xi}$ & 0 & & & & & & & & & & & \\
\hline$\underset{\mathrm{E}}{\mathrm{E}}$ & 35 & & & & & & 12 & 10 & 9 & 8 & 9 & 567 \\
\hline ర్ & 70 & & & & & 15 & 14 & 13 & 12 & 12 & 14 & 504 \\
\hline$\coprod_{\infty}^{\mathbb{\infty}}$ & 105 & & & & 20 & 18 & 17 & 17 & 17 & 17 & 20 & 437 \\
\hline$\frac{5}{0}$ & 140 & & & 25 & 23 & 22 & 21 & 21 & 21 & 22 & 26 & 381 \\
\hline 도 & 175 & & & 28 & 27 & 26 & 25 & 25 & 26 & 27 & 32 & 337 \\
\hline "艹 & 210 & & & 32 & 30 & 30 & 30 & 30 & 30 & 31 & 38 & 302 \\
\hline 오 & 245 & & 51 & 36 & 35 & 34 & 34 & 34 & 35 & 36 & 43 & 274 \\
\hline 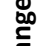 & 280 & & 42 & 40 & 40 & 39 & 39 & 39 & 39 & 41 & 48 & 252 \\
\hline $\begin{array}{l}\bar{\pi} \\
\stackrel{4}{1}\end{array}$ & 315 & & 46 & 44 & 44 & 43 & 43 & 43 & 44 & 46 & 54 & 235 \\
\hline$\stackrel{0}{5}$ & 350 & & 51 & 50 & 48 & 48 & 48 & 48 & 49 & 50 & 59 & 221 \\
\hline
\end{tabular}

Fig. 5.13: Navigation error relative to the LCT on the coarse grid using radiometric measurements to a single LCT at PDI, throttle-down, and landing. Values are in meters $3-\boldsymbol{\sigma}$. 
The fine grid essentially zooms in on the locations near the landing site as highlighted by a bold border in the results of the coarse grid. The results for the fine grid are shown in Fig. 5.14. There are two important observations that can be drawn from the results of the fine grid. First, the optimal location for an LCT is approximately $2 \mathrm{~km}$ up-range from the landing site and $2 \mathrm{~km}$ from the ground track. Second, as long as the LCT is placed no closer than this to the landing site, the accuracy of the LCT placement is not important (e.g., an LCT placed $5 \mathrm{~km}$ up-range and $5 \mathrm{~km}$ off-track performs nearly as well).

The sensitivity of relative navigation to key study parameters for the coarse grid are shown in Fig. 5.15. Navigation performance is again insensitive to variations in the range and range-rate measurement accuracies and the initial covariance of the lander and LCT position errors. The main effect of changing the visibility constraint is seen at PDI where more or fewer LCTs are made visible by the constraint. This effect is illustrated in Fig. 5.16 and Fig. 5.17. The map-tie error has no effect on the results of this study because the reference site location is directly observable. Finally, extending radiometric tracking before the deorbit burn proves significantly beneficial only at PDI when LCTs have not yet been visible on the descent trajectory.

The sensitivity of radiometric navigation using an LCT on the coarse grid to the descent trajectory type is displayed in Fig. 5.18. For the most part, the performance of navigation relative to beacons far from the landing site was not significantly affected by the trajectory type. Exceptions occur at throttle-down and HDA because the times at which the velocimeter becomes available (at $2 \mathrm{~km}$ altitude) and the altimeter model improves (at 500 m range) vary between trajectories. Velocimeter measurements for the "Stretch" trajectory are not available at throttle-down, causing significantly higher navigation errors. Also, because the "Sensor" trajectory enters the range where the altimeter model improves before throttle-down, the results for that trajectory are somewhat better that the others.

The sensitivity of the navigation performance to the trajectory type at HDA and throttle-down was found to be much more significant on the fine grid. The results for the fine grid on all eight trajectories are shown with a common color scale in Fig. 5.19. To 


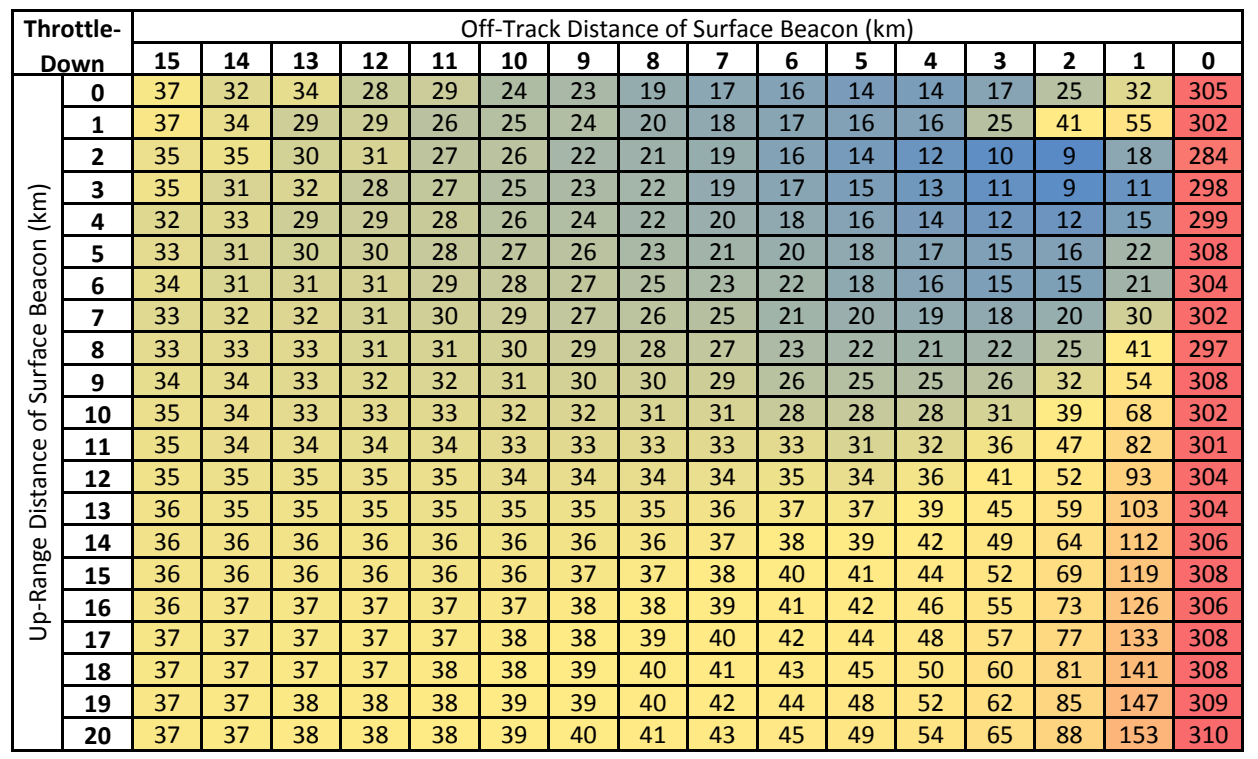

\begin{tabular}{|c|c|c|c|c|c|c|c|c|c|c|c|c|c|c|c|c|c|}
\hline \multirow{2}{*}{\multicolumn{2}{|c|}{ Landing }} & \multicolumn{16}{|c|}{ Off-Track Distance of Surface Beacon $(\mathrm{km})$} \\
\hline & & 15 & 14 & 13 & 12 & 11 & 10 & 9 & 8 & 7 & 6 & 5 & 4 & 3 & 2 & 1 & 0 \\
\hline \multirow{21}{*}{ 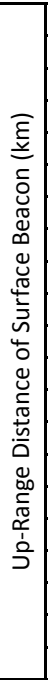 } & 0 & 8 & 8 & 8 & 8 & 7 & 7 & 7 & 6 & 6 & 5 & 5 & 5 & 5 & 6 & 5 & 69 \\
\hline & 1 & 8 & 8 & 8 & 7 & 7 & 7 & 7 & 6 & 6 & 5 & 5 & 5 & 4 & 5 & 5 & 194 \\
\hline & 2 & 8 & 8 & 8 & 7 & 7 & 7 & 6 & 6 & 5 & 5 & 5 & 5 & 4 & 4 & 4 & 246 \\
\hline & 3 & 8 & 8 & 7 & 7 & 7 & 6 & 6 & 6 & 5 & 5 & 5 & 5 & 5 & 4 & 5 & 253 \\
\hline & 4 & 8 & 8 & 7 & 7 & 7 & 6 & 6 & 6 & 6 & 5 & 5 & 5 & 5 & 5 & 6 & 245 \\
\hline & 5 & 8 & 7 & 7 & 7 & 6 & 6 & 6 & 6 & 6 & 6 & 5 & 5 & 5 & 6 & 7 & 272 \\
\hline & 6 & 8 & 7 & 7 & 7 & 6 & 6 & 6 & 6 & 6 & 6 & 5 & 5 & 6 & 6 & 9 & 278 \\
\hline & 7 & 7 & 7 & 7 & 7 & 6 & 6 & 6 & 6 & 6 & 6 & 6 & 6 & 6 & 7 & 11 & 285 \\
\hline & 8 & 7 & 7 & 7 & 7 & 7 & 6 & 6 & 6 & 6 & 6 & 6 & 6 & 6 & 7 & 12 & 282 \\
\hline & 9 & 7 & 7 & 7 & 7 & 7 & 6 & 6 & 6 & 6 & 6 & 6 & 6 & 7 & 8 & 13 & 291 \\
\hline & 10 & 7 & 7 & 7 & 7 & 7 & 6 & 6 & 6 & 6 & 6 & $\overline{6}$ & 6 & 7 & 8 & 14 & 286 \\
\hline & 11 & 7 & 7 & 7 & 7 & 7 & 6 & 6 & 6 & 6 & 6 & 6 & 7 & 7 & 9 & 15 & 285 \\
\hline & 12 & 7 & 7 & 7 & 7 & 7 & 6 & 6 & 6 & 6 & 6 & 6 & 7 & 8 & 9 & 16 & 288 \\
\hline & 13 & 8 & 7 & 7 & 7 & 7 & 7 & 6 & 6 & 6 & 7 & 7 & 7 & 8 & 10 & 17 & 287 \\
\hline & 14 & 7 & 7 & 7 & 7 & 7 & 7 & 7 & 7 & 7 & 7 & 7 & 7 & 8 & 10 & 17 & 289 \\
\hline & 15 & 7 & 7 & 7 & 7 & 7 & 7 & 7 & 7 & 7 & 7 & 7 & 7 & 8 & 11 & 18 & 291 \\
\hline & 16 & 7 & 7 & 7 & 7 & 7 & 7 & 7 & 7 & 7 & 7 & 7 & 8 & 9 & 11 & 19 & 289 \\
\hline & 17 & 7 & 7 & 7 & 7 & 7 & 7 & 7 & 7 & 7 & 7 & 7 & 8 & 9 & 11 & 20 & 290 \\
\hline & 18 & 8 & 7 & 7 & 7 & 7 & 7 & 7 & 7 & 7 & 7 & 8 & 8 & 9 & 12 & 21 & 291 \\
\hline & 19 & 8 & 8 & 7 & 7 & 7 & 7 & 7 & 7 & 7 & 7 & 8 & 8 & 9 & 12 & 22 & 291 \\
\hline & 20 & 8 & 8 & 8 & 7 & 7 & 7 & 7 & 7 & 7 & 8 & 8 & 9 & 10 & 13 & 23 & 292 \\
\hline
\end{tabular}

Fig. 5.14: Navigation results relative to an LCT on the fine grid using radiometric measurements to a single LCT at Throttle-down and landing. Values are in meters $3-\sigma$. 


\begin{tabular}{|c|c|c|c|c|c|c|}
\hline \multirow[b]{2}{*}{ Parameter } & \multirow[b]{2}{*}{$\begin{array}{c}\text { Baseline } \\
(3-\sigma)\end{array}$} & \multirow[b]{2}{*}{$\begin{array}{l}\text { Value } \\
(3-\sigma)\end{array}$} & \multicolumn{4}{|c|}{ Average Effect on Beacon-Relative Position Error of Lander } \\
\hline & & & $\begin{array}{c}\text { PDI } \\
\text { (visible) }\end{array}$ & \begin{tabular}{|c|} 
PDI \\
(not visible) \\
\end{tabular} & Throttle-Down & Landing \\
\hline Range Bias/Noise & $35 \mathrm{~ns}$ & & $\begin{array}{l}-3.1 \mathrm{~m}(-4.4 \%) \\
+2.2 \mathrm{~m}(+2.9 \%)\end{array}$ & $\begin{array}{ll}-0.4 \mathrm{~m} & (0.0 \%) \\
+0.0 \mathrm{~m} & (0.0 \%)\end{array}$ & $\begin{array}{ll}-0.4 \mathrm{~m} & (-0.6 \%) \\
+0.1 \mathrm{~m} & (+0.2 \%)\end{array}$ & $\begin{array}{ll}-0.3 \mathrm{~m} & (-0.7 \%) \\
+0.1 \mathrm{~m} & (+0.2 \%)\end{array}$ \\
\hline Doppler Bias/Noise & $0.5 \mathrm{~mm} / \mathrm{s}$ & $\begin{array}{c}0.05 \mathrm{~mm} / \mathrm{s} \\
5 \mathrm{~mm} / \mathrm{s}\end{array}$ & $\begin{array}{l}-0.2 \mathrm{~m}(-0.3 \%) \\
+2.7 \mathrm{~m}(+4.7 \%)\end{array}$ & \begin{tabular}{|cc}
$-0.8 m$ & $(-0.1 \%)$ \\
$+35.3 m$ & $(+2.1 \%)$ \\
\end{tabular} & $\begin{array}{l}-0.1 \mathrm{~m} \\
+1.0 .2 \%) \\
+1.8 \mathrm{~m}\end{array}$ & $\begin{array}{cc}0.0 \mathrm{~m} & (-0.3 \%) \\
+0.8 \mathrm{~m} & (+5.7 \%)\end{array}$ \\
\hline $\begin{array}{c}\text { Elevation Constraint for } \\
\text { Visibility }\end{array}$ & 10 deg & $\begin{array}{l}1 \mathrm{deg} \\
20 \mathrm{deg}\end{array}$ & \multicolumn{2}{|c|}{$\begin{array}{c}\text { more beacons visible } \\
\text { less beacons visible }\end{array}$} & $\begin{array}{l}-2.9 \mathrm{~m}(-5.2 \%) \\
+2.1 \mathrm{~m}(+3.4 \%)\end{array}$ & $\begin{array}{l}-1.1 \mathrm{~m}(-4.5 \%) \\
+0.8 \mathrm{~m}(+1.9 \%)\end{array}$ \\
\hline Map Tie & $100 \mathrm{~m}$ & $30 \mathrm{~m}$ & $-0.1 \mathrm{~m}(-0.2 \%)$ & $-0.4 \mathrm{~m} \quad(0.0 \%)$ & $-1.2 \mathrm{~m}(-1.7 \%)$ & $-0.6 \mathrm{~m}(-2.1 \%)$ \\
\hline Initial Lander Cov & $1500 \mathrm{~m}$ & $110 \mathrm{~m}$ & $-0.6 \mathrm{~m}(-2.3 \%)$ & $-50.2 \mathrm{~m}(-3.7 \%)$ & $-3.7 \mathrm{~m}(-8.3 \%)$ & $-4.3 \mathrm{~m}(-13.2 \%)$ \\
\hline Tracking Before Deorbit & On & Off & $+7.1 m(+19.0 \%)$ & $+2431 m(+152.5 \%)$ & $+4.5 \mathrm{~m} \mathrm{(+4.3 \% )}$ & $+4.9 \mathrm{~m} \mathrm{(+12.8 \% )}$ \\
\hline Avg. Position Error v & ith Baselin & Setup: & $59 \mathrm{~m}$ & $1659 \mathrm{~m}$ & $55 \mathrm{~m}$ & $31 \mathrm{~m}$ \\
\hline
\end{tabular}

Legend:

No significant Change

Significant Improvement

Significant Degradation

Fig. 5.15: Sensitivity of relative navigation results to key parameters for the coarse grid.

briefly summarize these results, the higher trajectories ("Stretch," "Peeky," and "Sensor") have better navigation performance than the lower trajectories ("Brakes," "Apollo-Like," and "Slanty"). The same exceptions mentioned in the coarse grid are found in the fine grid. 


\begin{tabular}{|c|c|c|c|c|c|c|c|c|c|c|c|c|}
\hline \multirow{2}{*}{\multicolumn{2}{|c|}{ PDI Error }} & \multicolumn{11}{|c|}{ Off-Track Location of Beacon (km) } \\
\hline & & \multirow{2}{*}{$\begin{array}{c}80 \\
1768 \\
\end{array}$} & \multirow{2}{*}{$\begin{array}{c}72 \\
1746 \\
\end{array}$} & \multirow{2}{*}{$\frac{64}{1720}$} & \multirow{2}{*}{$\begin{array}{c}\mathbf{5 6} \\
1690 \\
\end{array}$} & \multirow{2}{*}{$\begin{array}{c}\mathbf{4 8} \\
1654 \\
\end{array}$} & \multirow{2}{*}{$\begin{array}{c}40 \\
1613 \\
\end{array}$} & \multirow{2}{*}{$\begin{array}{c}32 \\
1569 \\
\end{array}$} & \multirow{2}{*}{$\begin{array}{c}24 \\
1524 \\
\end{array}$} & \multirow{2}{*}{$\begin{array}{c}16 \\
1483 \\
\end{array}$} & \multirow{2}{*}{$\begin{array}{c}8 \\
1451 \\
\end{array}$} & \multirow{2}{*}{$\frac{0}{1402}$} \\
\hline$\approx$ & 0 & & & & & & & & & & & \\
\hline$\stackrel{\underline{\underline{c}}}{\underline{\underline{*}}}$ & 35 & 1776 & 1754 & 1728 & 1697 & 1661 & 1621 & 1577 & 1534 & 1498 & 1479 & 1394 \\
\hline ธ & 70 & 1784 & 1761 & 1735 & 1704 & 1668 & 1628 & 1586 & 1546 & 1516 & 1512 & 1376 \\
\hline$\stackrel{\mathscr{ల}}{\mathscr{ల}}$ & 105 & 1792 & 1768 & 1742 & 1710 & 1674 & 1635 & 1594 & 106 & 137 & 247 & 496 \\
\hline 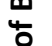 & 140 & 70 & 71 & 72 & 73 & 62 & 63 & 66 & 72 & 86 & 141 & 415 \\
\hline 등 & 175 & 56 & 56 & 56 & 55 & 56 & 57 & 59 & 63 & 74 & 117 & 359 \\
\hline 吾 & 210 & 52 & 51 & 51 & 51 & 52 & 53 & 54 & 58 & 67 & 102 & 318 \\
\hline อ & 245 & 49 & 48 & 48 & 48 & 48 & 49 & 50 & 54 & 62 & 93 & 287 \\
\hline ט. & 280 & 47 & 46 & 46 & 45 & 45 & 45 & 46 & 49 & 56 & 86 & 263 \\
\hline$\underset{\mathbb{\pi}}{\mathbb{4}}$ & 315 & 47 & 46 & 45 & 45 & 44 & 44 & 44 & 45 & 51 & 78 & 243 \\
\hline$\stackrel{\circ}{\supset}$ & 350 & 46 & 45 & 44 & 43 & 42 & 42 & 42 & 44 & 50 & 75 & 228 \\
\hline
\end{tabular}

\begin{tabular}{|c|c|c|c|c|c|c|c|c|c|c|c|c|}
\hline \multirow{2}{*}{\multicolumn{2}{|c|}{$\begin{array}{c}\text { PDI } \\
\text { Sensitivity }\end{array}$}} & \multicolumn{11}{|c|}{ Off-Track Location of Beacon (km) } \\
\hline & & 80 & 72 & 64 & 56 & 48 & 40 & 32 & 24 & 16 & 8 & 0 \\
\hline \multirow{11}{*}{ 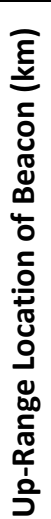 } & 0 & & & & & & & & & $-3 \%$ & $-3 \%$ & $-2 \%$ \\
\hline & 35 & & & & & & $-2 \%$ & $-2 \%$ & $-2 \%$ & $-3 \%$ & $-3 \%$ & $-3 \%$ \\
\hline & 70 & & & & & $-2 \%$ & $-2 \%$ & $-2 \%$ & $-3 \%$ & $-3 \%$ & $-3 \%$ & $-3 \%$ \\
\hline & 105 & & & & $-1 \%$ & $-2 \%$ & $-2 \%$ & $-2 \%$ & $-93 \%$ & $-91 \%$ & $-84 \%$ & $-65 \%$ \\
\hline & 140 & & & $-96 \%$ & $-96 \%$ & $-96 \%$ & $-96 \%$ & $-96 \%$ & $-96 \%$ & $-95 \%$ & $-91 \%$ & $-70 \%$ \\
\hline & 175 & & & $-97 \%$ & $-97 \%$ & $-97 \%$ & $-97 \%$ & $-96 \%$ & $-96 \%$ & $-96 \%$ & $-93 \%$ & $-74 \%$ \\
\hline & 210 & & & $-97 \%$ & $-97 \%$ & $-97 \%$ & $-97 \%$ & $-97 \%$ & $-97 \%$ & $-96 \%$ & $-94 \%$ & $-77 \%$ \\
\hline & 245 & & $-97 \%$ & $-97 \%$ & $-97 \%$ & $-24 \%$ & $-24 \%$ & $-24 \%$ & $-24 \%$ & $-24 \%$ & $-24 \%$ & $-5 \%$ \\
\hline & 280 & & $-98 \%$ & $-26 \%$ & $-27 \%$ & $-14 \%$ & $-15 \%$ & $-17 \%$ & $-6 \%$ & $-6 \%$ & $-6 \%$ & $-5 \%$ \\
\hline & 315 & & $-10 \%$ & $-8 \%$ & $-8 \%$ & $-6 \%$ & $-7 \%$ & $-8 \%$ & $-10 \%$ & $-10 \%$ & $-8 \%$ & $-5 \%$ \\
\hline & 350 & & $-13 \%$ & $-12 \%$ & $-3 \%$ & $-3 \%$ & $-4 \%$ & $-5 \%$ & $-5 \%$ & $-4 \%$ & $-4 \%$ & $-4 \%$ \\
\hline
\end{tabular}

Fig. 5.16: Upper Chart: The navigation results at PDI relative to a single surface beacon on the coarse grid when the visibility constraint is lowered to 1 degree. Compare with Fig. 5.13. Lower Chart: The percent change in the navigation results when the visibility constraint is lowered from 10 degrees to 1 degree. Five different areas are outlined. The green and grey areas on the left include beacons that are now visible at some point during the final descent. The grey area is visible at or before PDI. Beacons in the blue and yellow area at the bottom have increased tracking time before PDI. The beacons in the red area in the middle are now visible at PDI. The beacons in the blue area at the top saw no change in visibility at PDI. 


\begin{tabular}{|c|c|c|c|c|c|c|c|c|c|c|c|c|}
\hline \multirow{2}{*}{\multicolumn{2}{|c|}{ PDI Error }} & \multicolumn{11}{|c|}{ Off-Track Location of Beacon $(\mathrm{km})$} \\
\hline & & \multirow[t]{2}{*}{80} & \multirow[t]{2}{*}{72} & \multirow[t]{2}{*}{64} & \multirow[t]{2}{*}{56} & \multirow[t]{2}{*}{48} & \multirow[t]{2}{*}{40} & \multirow[t]{2}{*}{32} & \multirow[t]{2}{*}{24} & \multirow[t]{2}{*}{16} & \multirow[t]{2}{*}{8} & \multirow{2}{*}{$\frac{0}{1463}$} \\
\hline \multirow{11}{*}{ 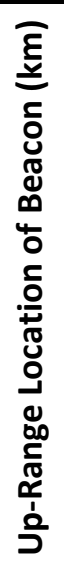 } & 0 & & & & & & & & & & & \\
\hline & 35 & & & & & & & & & 1583 & 1563 & 1461 \\
\hline & 70 & & & & & & & & 1626 & 1602 & 1593 & 1452 \\
\hline & 105 & & & & & & & & 1634 & 1617 & 1621 & 1441 \\
\hline & 140 & & & & & & & 1673 & 1647 & 1640 & 1650 & 1439 \\
\hline & 175 & & & & & & & 1684 & 1663 & 1666 & 1666 & 1441 \\
\hline & 210 & & & & & & & 1696 & 1681 & 1692 & 1643 & 1442 \\
\hline & 245 & & & & & & & 1704 & 1695 & 1724 & 1581 & 1449 \\
\hline & 280 & & & & & & & 66 & 69 & 78 & 112 & 289 \\
\hline & 315 & & & & & & & 54 & 57 & 57 & 86 & 265 \\
\hline & 350 & & & & & & & 48 & 49 & 55 & 79 & 246 \\
\hline
\end{tabular}

\begin{tabular}{|c|c|c|c|c|c|c|c|c|c|c|c|c|}
\hline \multirow{2}{*}{\multicolumn{2}{|c|}{\begin{tabular}{|c|} 
PDI \\
Sensitivity \\
\end{tabular}}} & \multicolumn{11}{|c|}{ Off-Track Location of Beacon (km) } \\
\hline & & 80 & 72 & 64 & 56 & 48 & 40 & 32 & 24 & 16 & 8 & 0 \\
\hline \multirow{11}{*}{ 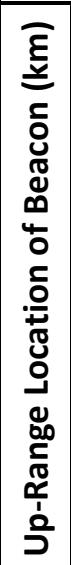 } & 0 & & & & & & & & & & & $2 \%$ \\
\hline & 35 & & & & & & & & & $3 \%$ & $3 \%$ & $2 \%$ \\
\hline & 70 & & & & & & & & $3 \%$ & $3 \%$ & $3 \%$ & $2 \%$ \\
\hline & 105 & & & & & & & & $2 \%$ & $3 \%$ & $2 \%$ & $3 \%$ \\
\hline & 140 & & & & & & & $2 \%$ & $2 \%$ & $3 \%$ & $2 \%$ & $3 \%$ \\
\hline & 175 & & & & & & & $2 \%$ & $2 \%$ & $3 \%$ & $1 \%$ & $3 \%$ \\
\hline & 210 & & & & & & & $2 \%$ & $2 \%$ & $2 \%$ & $1 \%$ & $2 \%$ \\
\hline & 245 & & & & & & & $2484 \%$ & $2301 \%$ & $2022 \%$ & $1198 \%$ & $377 \%$ \\
\hline & 280 & & & & & & & $19 \%$ & $35 \%$ & $30 \%$ & $22 \%$ & $5 \%$ \\
\hline & 315 & & & & & & & $13 \%$ & $14 \%$ & $1 \%$ & $2 \%$ & $4 \%$ \\
\hline & 350 & & & & & & & $10 \%$ & $7 \%$ & $6 \%$ & $2 \%$ & $3 \%$ \\
\hline
\end{tabular}

Fig. 5.17: Upper Chart: The navigation results at PDI relative to a single surface beacon on the coarse grid when the visibility constraint is raised to 20 degrees. Compare with Fig. 5.13. Lower Chart: The percent change in the navigation results when the visibility constraint is raised from 10 degrees to 20 degrees. Six different areas are outlined. The white area on the left was not visible before or after the change. The beacons in the green and grey areas in the center have completely lost visual contact with the lander during the final descent. The grey area was once visible at or before PDI. Beacons in the blue and yellow area at the bottom right have less tracking time before PDI. The beacons in the red line in the middle are now not visible at PDI. The beacons in the blue area at the top right saw no change in visibility at PDI. 


\begin{tabular}{|c|c|c|c|c|c|}
\hline \multirow[b]{2}{*}{ Trajectory \# } & \multicolumn{4}{|c|}{ Average Effect on Beacon-Relative Position Error of Lander } & \multirow{3}{*}{$\begin{array}{c}\text { Change in \# } \\
\text { Beacons } \\
\text { Visibile }\end{array}$} \\
\hline & $\begin{array}{c}\text { PDI } \\
\text { (visible) }\end{array}$ & $\begin{array}{c}\text { PDI } \\
\text { (not visible) } \\
\end{array}$ & Throttle-Down & Landing & \\
\hline Baseline & $59 \mathrm{~m} \mathrm{3- \sigma}$ & $1659 \mathrm{~m} \mathrm{3- \sigma}$ & 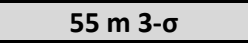 & 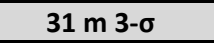 & \\
\hline Brakes & $-2.7 \mathrm{~m}(-3.6 \%)$ & $-0.8 \mathrm{~m}(-0.1 \%)$ & $-1.7 \mathrm{~m}(-2.1 \%)$ & $-1.8 \mathrm{~m}(-4.5 \%)$ & -1 \\
\hline Apollo-Like & $-3.2 \mathrm{~m}(-4.5 \%)$ & $-0.3 \mathrm{~m}(0.0 \%)$ & $-2.3 \mathrm{~m}(-2.9 \%)$ & $-2.8 \mathrm{~m}(-8.2 \%)$ & -2 \\
\hline Slanty & $-2.8 \mathrm{~m}(-3.6 \%)$ & $-0.7 \mathrm{~m}(0.0 \%)$ & $-2.7 \mathrm{~m}(-4 \%)$ & $-2.2 \mathrm{~m}(-7.1 \%)$ & -2 \\
\hline Stretch & $-3.1 \mathrm{~m}(-4.4 \%)$ & $-0.4 \mathrm{~m}(0.0 \%)$ & $+64.8 \mathrm{~m} \mathrm{(+107.8 \% )}$ & $+1.8 \mathrm{~m} \mathrm{(+5.4 \% )}$ & +3 \\
\hline Peeky & $+0.0 \mathrm{~m}(0.0 \%)$ & $0.0 \mathrm{~m}(0.0 \%)$ & $+1.2 \mathrm{~m} \mathrm{(+2.2 \% )}$ & $+1.0 \mathrm{~m}(+3.6 \%)$ & +2 \\
\hline Sensor & $-3.2 \mathrm{~m}(-4.3 \%)$ & $-0.3 \mathrm{~m}(0.0 \%)$ & $-8.7 \mathrm{~m}(-15.9 \%)$ & $+3.1 \mathrm{~m} \mathrm{(+9.0 \% )}$ & +5 \\
\hline Cheapo & $0.0 \mathrm{~m}(0.0 \%)$ & $+0.0 \mathrm{~m}(0.0 \%)$ & $+0.2 \mathrm{~m} \mathrm{(+0.8 \% )}$ & $+0.4 \mathrm{~m}(+0.7 \%)$ & 0 \\
\hline
\end{tabular}

Fig. 5.18: Sensitivity of relative navigation results on the coarse grid to the trajectory type. 

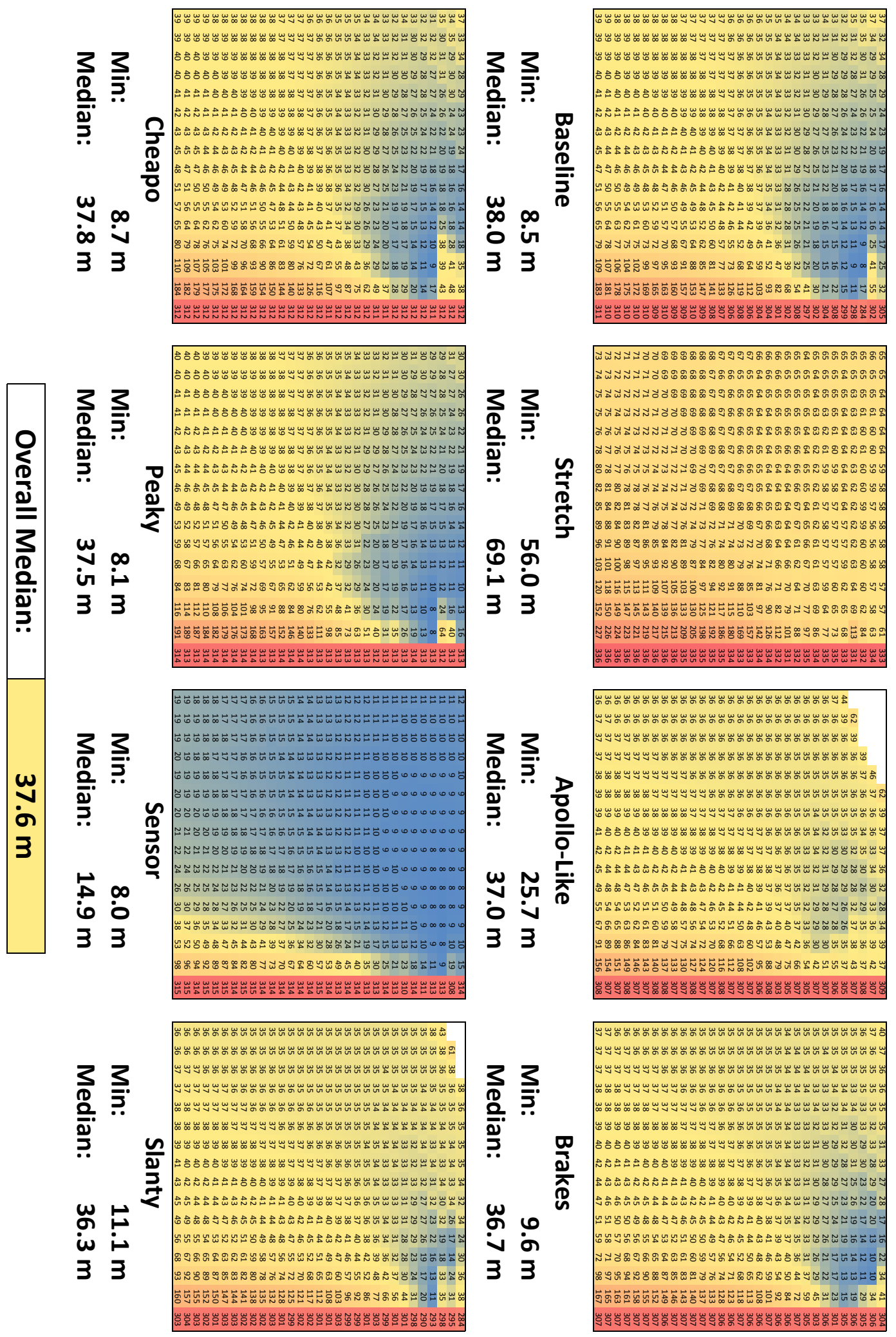

Fig. 5.19: Navigation results relative to an LCT on the fine grid for all Trajectories at throttle-down. Compare with Fig. 5.14 and 5.20. 

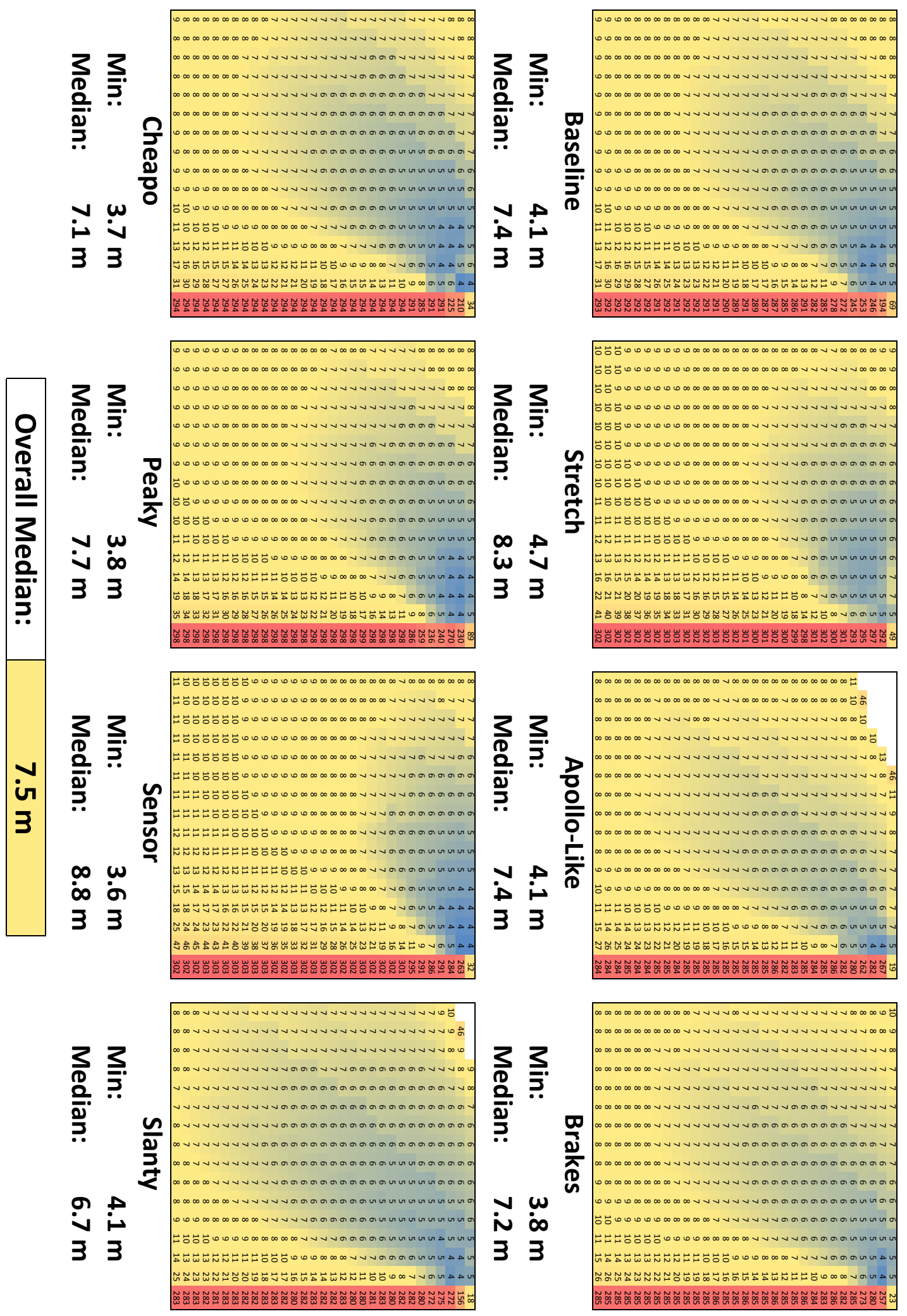

Fig. 5.20: Navigation results relative to an LCT on the fine grid for all Trajectories at landing. Compare with Fig. 5.14 and 5.19. 


\section{Chapter 6}

\section{Conclusions}

In this research, a linear covariance simulation was used to evaluate terrain-relative navigation (TRN) and radiometric beacon-relative navigation against navigation requirements at four key locations along the descent trajectory: PDI, throttle-down, HDA and landing. The goal was to show that TRN is more flexible and reliable for lunar landings than surface and orbiting beacon-relative navigation $(<100 \mathrm{~m} 3-\sigma)$. Sensitivity studies were conducted on both navigation techniques to evaluate their flexibility and reliability. Even though the equivalent of well over five thousand Monte Carlo studies were conducted to support this paper, the total required CPU time was minimal given that on average, evaluating each configuration using linear covariance took less than 2 minutes on a common desktop computer with a single processor. If these studies would have been carried out back-to-back, the total CPU time would have been approximately one week. The time that the equivalent Monte Carlo studies would require using the same computer platform likely would be measured in years.

The TRN analysis showed that any operating range of the TRN could easily support the navigation requirements at all four key locations along the descent trajectory. It was observed that the time at which the TRN sensor is turned off has a much more significant effect than the time at which it turns on and that later TRN cutoff times provide better navigation performance. The effect of using TRN measurements near the landing site where higher resolution maps are available significantly lowers the navigation errors well below the requirements. Simulations of outpost class missions near the lunar equator (a worst-case scenario), using maps that will be acquired by the Lunar Reconnaissance Orbiter (LRO), showed that if the 3-dimensional LRO map resolution was improved by an order of magnitude near the landing site, TRN could support a precision landing at the equator. 
Radiometric range and range-rate measurements relative to one or two orbiting beacons or one surface beacon were evaluated in the second stage of the analysis. One orbiting beacon was able to support navigation accuracies on the order of $200 \mathrm{~m} \mathrm{3-} \sigma$, which can support PDI and throttle-down, but not HDA and landing. Adding a second orbiting beacon provided much better results that were less sensitive to changes in the configuration, but this constellation also could not support HDA and landing unless the inertial error of the landing site (the map-tie error) is reduced from $100 \mathrm{~m} \mathrm{3-} \sigma$ to less than $60 \mathrm{~m} \mathrm{3-} \sigma$. Finally, surface beacons provide the most powerful radiometric measurements so long as they are not located directly under the path of the lander as it approaches the landing site. For this reason, a well-placed beacon can easily support all phases of the descent.

Based upon the results of these studies, there are multiple configurations that would be able to meet the requirements for a precision landing. First, surface-relative navigation using TRN or a surface beacon can easily support PDI, throttle-down, HDA, and landing if they are used early in the descent trajectory and, in the case of the surface beacon, if its position relative to the landing site is well known. Radiometric navigation using two orbiting beacons can also meet all requirements if the map-tie error is reduced. It is important to note that either late TRN or a surface beacon in the proximity of the landing site could be used to support a high-precision landing $(<10 \mathrm{~m} 3-\sigma)$. In this case, PDI would need to be supported by one of the methods discussed in this paper. Operationally, if the lander was returning to a previously visited landing site, this navigation accuracy might eliminate the need for autonomous hazard detection.

It is thus concluded that both TRN and radiometric navigation can be used to support the navigation requirements at all four key locations along the descent trajectory. Concerning flexibility and reliability, it is concluded that, first, TRN can support precision lunar landings anytime and anywhere if the resolution of LRO maps near landing sites at lower latitudes is significantly increased. Second, if the accuracy of the maps was increased (which would reduce the map-tie error), two coplanar orbiting beacons could be positioned in their orbits such that they would be able to support such a landing. Third, unless surface beacons 
become relatively efficient to produce and deploy, which is unlikely, navigation relative to a surface beacon will not be adequately flexible. Thus, neither the current TRN technique nor the radiometric navigation techniques would be able to support the requirements for precision landings anytime and anywhere with the current requirements for the LRO maps. The TRN navigation, however, is still much more flexible than radiometric navigation because it alone is able to support navigation at all four key locations along the descent trajectory when the landing site is at a high enough latitude. From the results of this research, it is apparent that radiometric navigation is best suited for returning to a previously-visited landing site and terrain-relative navigation is the best option for navigating to new landing sites. 


\section{Chapter 7 Future Work}

\subsection{Additional Terrain-Relative Navigation Work}

This research only investigated the use of an active TRN sensor. This type of sensor functions very well at the poles using maps of the accuracy hoped to be gained from the LRO and it is apparent that it functions much less accurately in the mid-latitudes where the 3-dimensional map resolution is much more sparse. It seems likely that a passive TRN sensor could more accurately support descent trajectories in the mid-latitudes where there is a much greater abundance of craters and other features on the surface of the moon for

optical tracking. Also, the LRO will produce optical images with $1 \mathrm{~m}$ resolution that could support a passive navigation system very well. A separate linear covariance analysis could investigate the utility of a passive sensor instead of an active sensor. The main difference in the model is that a passive sensor would provide angular measurements with limited range data, while the an active senor would provide much more accurate range measurements to each landmark.

\subsection{Additional Radiometric Navigation Work}

This research is not an exhaustive study of all the possible configurations for radiometric navigation. A number of additional studies that could be performed include an investigation of the navigation performance of the entire Lunar Network, which consists of two LCTs and two LRSs. Other types of beacons could be studied as well, including ground-based tracking stations such as the Deep Space Network, and halo orbiters placed at the Lagrange points between the earth and the moon and on the far side of the moon. 


\subsection{Guidance Implementation}

An obvious next step for this research is to incorporate a linearized version of the guidance law and the control dynamics into the linear covariance simulation to generate accurate dispersion results. This would enable a quantitative closed-loop analysis into the effect of navigation performance on the control dispersions and the fuel efficiency of the landing.

\subsection{Modeling Improvement}

It is a well-known fact that there is no end to increasing the fidelity of the mathematical models used in computer simulations. For this analysis, the areas in which the models can be improved include investigating a possible method by which a time-varying Markov process could be used to model the map resolution as the lander approaches the pole of the moon. This would much better capture the effect of the latitude/longitude-based grid of the 3-dimensional maps acquired by the LRO than the current two-tier system.

Another possible area for model improvement would be in implementing one-way pseudo-range and pseudo-Doppler measurements between the lander and the beacons when there is more than one visible. To accomplish this, the error of the clock onboard the lander would also need to be accounted for.

\subsection{Reduced-State Filter}

Though it is of low priority at this stage of the ALHAT analysis, the linear covariance simulation is very well equipped for testing the effect of reducing the number of states estimated onboard the filter. To do this, the uncertainties in all of the states that are removed from the filter would be replaced with additional white process noise. This task, however, is only important when the extended Kalman filter that will fly onboard the lander is being designed. Otherwise, it is assumed that the full-state filter will yield similar results as a well-tuned reduced-state filter and thus the need to identify unneeded states is a low priority. 


\section{References}

[1] Epp, C. D. and Smith, T. B., "The Autonomous Precision Landing and Hazard Detection and Avoidance Technology (ALHAT)," IEEE Aerospace Conference Proceedings, Institute of Electrical and Electronics Engineers, Big Sky, MT, 2007.

[2] Hoag, D. G., "The History of Apollo Onboard Guidance, Navigation, and Control," Journal of Guidance, Control, and Dynamics, Vol. 6, No. 1, 1982, pp. 4-13.

[3] Barton, G. H., Shepherd, S. W., and Brand, T. J., "Proposed Autonomous Lunar Navigation System," Proceedings of the Annual Rocky Mountain Guidance and Control Conference, American Astronautical Society, 1993, pp. 1717-1736, AAS 93-713.

[4] Paschall, S., Cohanim, B. E., Brady, T., and Sostaric, R., "A Self Contained Method for Safe \& Precise Lunar Landing," IEEE Aerospace Conference, Institute of Electrical and Electronics Engineers, Big Sky, MT, 2008.

[5] Schiesser, E., "Navigation Error Covariance Matrices for CEV and LSAM Lunar Orbit GN\&C Analysis," Informal memo, March 2006.

[6] Baker, J. D., "Lunar Reconnaissance Orbiter (LRO) Requirements," Tech. Rep. ESMD-RLEP-0010, National Aeronautics and Space Administration, November 2005.

[7] Geller, D. K. and Christensen, D. P., "Linear Covariance Analysis for Lunar Powered Descent and Landing," Submitted to AIAA Journal of Guidance, Control, and Dynamics.

[8] Davies, M. E., Abalakin, V. K., Bursa, M., Lieske, J. H., Morando, B., Morrison, D., Seidelmann, P. K., Sinclair, A. T., Yallop, B., and Tjuflin, Y. S., "Cartographic Corrdinates and Rotational Elements of the Planets and Satellites: 1994," Celestial Mechanics and Dynamical Astronomy, Vol. 63, No. 2, June 1995, pp. 127-148. 
[9] Battin, R. H., An Introduction to the Mathematics and Methods of Astrodynamics, American Institute of Aeronautics and Astronautics, Reston, VA, rev. ed., 1999.

[10] Curtis, H. D., Orbital Mechanics for Engineering Students, chap. 4, Elsevier Butterworth-Heinemann, Burlington, MA, 2005, pp. 149-191.

[11] Montenbruck, O. and Gill, E., Satellite Orbits: Models, Methods and Applications, chap. 7, Springer, New York, NY, 2005, pp. 236-238.

[12] Vallado, D. A., Fundamentals of Astrodynamics and Applications, chap. 11, Springer, New York, NY, 2007, pp. 827-931.

[13] Ely, T. A., "Constellations of Elliptical Inclined Lunar Orbits Providing Polar and Global Coverage," The Journal of the Astronautical Sciences, Vol. 54, No. 1, 2006, pp. 53-67.

[14] Ely, T. A., "Stable Constellations of Frozen Elliptical Inclined Lunar Orbits," The Journal of the Astronautical Sciences, Vol. 53, No. 3, 2005, pp. 301-316.

[15] Johnson, A. E. and Montgomery, J. F., "Overview of Terrain Relative Navigation Approaches for Precise Lunar Landing," IEEE Aerospace Conference, Institute of Electrical and Electronics Engineers, Big Sky, MT, 2008.

[16] Singh, L. and Lim, S., "On Lunar on-orbit Vision-Based Navigation: Terrain Mapping, Feature Tracking driven EKF," Guidance, Navigation and Control Conference and Exhibit, American Institute of Aeronautics and Astronautics, Honolulu, HI, August 2008 .

[17] NASA, "NASA‘s Lunar Communications \& Navigation Architecture," Technology Exchange Conference, November 2007.

[18] Ely, T. A., "Simplified Radiometric Measurement Models of the Lunar Network," Unpublished. 
[19] Kalman, R. E., "A New Approach to Linear Filtering and Prediction Problems," Transactions of the ASME - Journal of Basic Engineering, Vol. 82, 1960, pp. 35-45.

[20] Crassidis, J. L. and Junkins, J. L., Optimal Estimation of Dynamic Systems, Chapman \& Hall/CRC, Boca Raton, FL, 2004.

[21] Maybeck, P. S., Stochastic Models, Estimation, and Control, Vol. 1, Academic Press, New York, NY, 1979.

[22] Woffinden, D. C. and Geller, D. K., "Relative Angles-Only Navigation and Pose Estimation for Autonomous Orbital Rendezvous," Journal of Guidance, Control, and Dynamics, Vol. 30, No. 5, Sept-Oct 2007, pp. 1455-1469.

[23] Geller, D. K., "Linear Covariance Techniques for Orbital Rendezvous Analysis and Autonomous Onboard Mission Planning," Journal of Guidance, Control, and Dynamics, Vol. 29, No. 6, 2006, pp. 1404-1414.

[24] Pittelkau, M. E., "Rotation Vector in Attitude Estimation," Journal of Guidance, Control, and Dynamics, Vol. 26, No. 6, 2003, pp. 855-860.

[25] Lefferts, E. J., Markley, F. L., and Shuster, M., "Kalman Filtering for Spacecraft Estimation," Journal of Guidance, Control, and Dynamics, Vol. 5, No. 5, 1982, pp. 417429. 
Appendices 


\section{Appendix A}

\section{Matrix Partial Derivatives}

\section{A.1 Covariance Propagation Matrices}

The propagation of the navigation state $\dot{\hat{\boldsymbol{x}}}=\hat{\boldsymbol{f}}(\hat{\boldsymbol{x}}, \hat{\boldsymbol{u}}, \tilde{\boldsymbol{y}})$ is accomplished using Equations (4.50-4.57). The propagation of the navigation state covariance matrix in Equation (4.61) is accomplished using the following partial derivative and state process noise covariances: (for the order of the states, see Table 4.1.)

$$
\begin{aligned}
& \hat{F}_{\hat{x}}=\frac{\partial \hat{\boldsymbol{f}}}{\partial \hat{\boldsymbol{x}}}=\left(\begin{array}{ccc}
\hat{D}_{\hat{d}} & \hat{D}_{\hat{p}} & \hat{D}_{\hat{e}} \\
O_{47 \times 24} & -\operatorname{Diag}\left(\left[\frac{1}{\hat{\tau}_{1}}, \frac{1}{\hat{\tau}_{2}}, \ldots, \frac{1}{\hat{\tau}_{47}}\right]\right) & O_{47 \times 7} \\
O_{7 \times 24} & O_{7 \times 47} & -\operatorname{Diag}\left(\left[\frac{\left|\hat{\boldsymbol{v}}^{i}\right|}{\hat{d}_{1}}, \frac{\left|\hat{\boldsymbol{v}}^{i}\right|}{\hat{d}_{2}}, \ldots, \frac{\left|\hat{\boldsymbol{v}}^{i}\right|}{\hat{d}_{7}}\right]\right)
\end{array}\right) \\
& \hat{S}_{\eta}=\left(\begin{array}{ccc}
\hat{S}_{\eta_{d}} & O_{24 \times 47} & O_{24 \times 7} \\
O_{47 \times 24} & O_{47 \times 47} & O_{47 \times 7} \\
O_{7 \times 24} & O_{7 \times 47} & O_{7 \times 7}
\end{array}\right) \\
& \hat{S}_{w}=\left(\begin{array}{ccc}
\hat{S}_{a_{d}} & O_{24 \times 47} & O_{24 \times 7} \\
O_{47 \times 24} & -\operatorname{Diag}\left(\left[\hat{\sigma}_{p_{1}}^{2}, \hat{\sigma}_{p_{2}}^{2}, \ldots, \hat{\sigma}_{p_{47}}^{2}\right]\right) & O_{47 \times 7} \\
O_{7 \times 24} & O_{7 \times 47} & -\operatorname{Diag}\left(\left[\hat{\sigma}_{\varepsilon_{1}}^{2}, \hat{\sigma}_{\varepsilon_{2}}^{2}, \ldots, \hat{\sigma}_{\varepsilon_{7}}^{2}\right]\right)
\end{array}\right)
\end{aligned}
$$

where

$$
\hat{D}_{\hat{d}}=\left(\begin{array}{ccc}
\hat{D}_{\hat{d}_{\ell}} & O_{9 \times 12} & O_{9 \times 3} \\
O_{12 \times 9} & \hat{D}_{\hat{d}_{l r s}} & O_{12 \times 3} \\
O_{3 \times 9} & O_{9 \times 12} & {\left[\boldsymbol{\omega}_{\text {moon }}^{i} \times\right]}
\end{array}\right)
$$




$$
\begin{aligned}
& \hat{D}_{\hat{d}_{\ell}}=\left(\begin{array}{ccc}
O_{3 \times 3} & I_{3 \times 3} & O_{3 \times 3} \\
\frac{\partial \hat{\boldsymbol{a}}_{\text {grav }}^{i}}{\partial \hat{\boldsymbol{r}}^{i}} & O_{3 \times 3} & -\hat{\mathcal{T}}_{\ell}^{i}\left[\tilde{\boldsymbol{a}}_{\text {accel }}^{\ell} \times\right] \\
O_{3 \times 3} & O_{3 \times 3} & -\left[\tilde{\boldsymbol{\omega}}^{\ell} \times\right]
\end{array}\right), \quad \hat{D}_{\hat{d}_{l r s}}=\left(\begin{array}{cccc}
O_{3 \times 3} & O_{3 \times 3} & I_{3 \times 3} & O_{3 \times 3} \\
O_{3 \times 3} & O_{3 \times 3} & O_{3 \times 3} & I_{3 \times 3} \\
\frac{\partial \hat{\boldsymbol{a}}_{\text {grav }}^{i}}{\partial \hat{\boldsymbol{r}}^{i}} & O_{3 \times 3} & O_{3 \times 3} & O_{3 \times 3} \\
O_{3 \times 3} & \frac{\partial \hat{\boldsymbol{a}}_{\text {grav }}^{i}}{\partial \hat{\boldsymbol{r}}^{i}} & O_{3 \times 3} & O_{3 \times 3}
\end{array}\right) \\
& \hat{D}_{\hat{p}}=\left(\begin{array}{ccccccc}
O_{3 \times 3} & O_{3 \times 3} & O_{3 \times 3} & O_{3 \times 3} & O_{3 \times 3} & O_{3 \times 3} & O_{3 \times 29} \\
I_{3 \times 3} & \hat{\mathcal{T}}_{\ell}^{i} \operatorname{Diag}\left(\tilde{\boldsymbol{a}}_{a c c e l}^{\ell}\right) & \hat{\mathcal{T}}_{\ell}^{i}\left[\tilde{\boldsymbol{a}}_{a c c e l}^{\ell} \times\right] & O_{3 \times 3} & O_{3 \times 3} & O_{3 \times 3} & O_{3 \times 29} \\
O_{3 \times 3} & O_{3 \times 3} & O_{3 \times 3} & I_{3 \times 3} & \operatorname{Diag}\left(\tilde{\boldsymbol{\omega}}^{\ell}\right) & {\left[\tilde{\boldsymbol{\omega}}^{\ell} \times\right]} & O_{3 \times 29} \\
O_{12 \times 3} & O_{12 \times 3} & O_{12 \times 3} & O_{12 \times 3} & O_{12 \times 3} & O_{12 \times 3} & O_{12 \times 29} \\
O_{3 \times 3} & O_{3 \times 3} & O_{3 \times 3} & O_{3 \times 3} & O_{3 \times 3} & O_{3 \times 3} & O_{3 \times 29}
\end{array}\right) \\
& \hat{D}_{\hat{e}}=\left(\begin{array}{cc}
O_{3 \times 4} & O_{3 \times 3} \\
O_{3 \times 4} & I_{3 \times 3} \\
O_{3 \times 4} & O_{3 \times 3} \\
O_{12 \times 4} & O_{12 \times 3} \\
O_{3 \times 4} & O_{3 \times 3}
\end{array}\right), \quad \hat{S}_{\eta_{d}}=\left(\begin{array}{ccccc}
O_{3 \times 3} & O_{3 \times 3} & O_{3 \times 3} & O_{3 \times 12} & O_{3 \times 3} \\
O_{3 \times 3} & \hat{S}_{\eta_{a c c e l}} & O_{3 \times 3} & O_{3 \times 12} & O_{3 \times 3} \\
O_{3 \times 3} & O_{3 \times 3} & \hat{S}_{\eta_{g y r o}} & O_{3 \times 12} & O_{3 \times 3} \\
O_{12 \times 3} & O_{12 \times 3} & O_{12 \times 3} & O_{12 \times 12} & O_{12 \times 3} \\
O_{3 \times 3} & O_{3 \times 3} & O_{3 \times 3} & O_{3 \times 12} & O_{3 \times 3}
\end{array}\right) \\
& \hat{S}_{a_{d}}=\left(\begin{array}{ccccc}
O_{3 \times 3} & O_{3 \times 3} & O_{3 \times 3} & O_{3 \times 12} & O_{3 \times 3} \\
O_{3 \times 3} & \hat{S}_{a} & O_{3 \times 3} & O_{3 \times 12} & O_{3 \times 3} \\
O_{3 \times 3} & O_{3 \times 3} & O_{3 \times 3} & O_{3 \times 12} & O_{3 \times 3} \\
O_{12 \times 3} & O_{12 \times 3} & O_{12 \times 3} & O_{12 \times 12} & O_{12 \times 3} \\
O_{3 \times 3} & O_{3 \times 3} & O_{3 \times 3} & O_{3 \times 12} & O_{3 \times 3}
\end{array}\right)
\end{aligned}
$$

\section{A.2 Measurement Sensitivity Matrices}

The measurement sensitivity matrix for the star-camera is given by

$$
\hat{H}_{\hat{x}}^{\text {starcam }}=\left(O_{3 \times 6}, \hat{\mathcal{T}}_{\ell}^{\text {starcam }}, O_{3 \times 15}, O_{3 \times 18}, I_{3 \times 3}, O_{3 \times 26}, O_{3 \times 7}\right)
$$


The flight computer's value of the star-camera measurement covariance is given by

$$
\hat{R}_{\nu}^{\text {starcam }}=\operatorname{Diag}\left(\left[\hat{\sigma}_{\text {starcam }_{x}}^{2}, \hat{\sigma}_{\text {starcam }_{y}}^{2}, \hat{\sigma}_{\text {starcam }_{z}}^{2}\right]\right)
$$

The measurement sensitivity matrix for the altimeter is given by

$$
\hat{H}_{\hat{x}}^{a l t}=\left(\left(\hat{\boldsymbol{r}}^{i}\right)^{T} /\left|\hat{\boldsymbol{r}}^{i}\right|, O_{1 \times 21}, O_{1 \times 21}, 1,\left(\left|\hat{\boldsymbol{r}}^{i}\right|-\rho_{\text {moon }}\right), O_{1 \times 24}, O_{1 \times 3}, 1, O_{1 \times 3}\right)
$$

The flight computer's value of the altimeter's measurement covariance is simply given by

$$
\hat{R}_{\nu}^{a l t}=\hat{\sigma}_{\nu_{a l t}}^{2}
$$

The measurement sensitivity matrix for the velocimeter is given by

$$
\begin{array}{r}
\hat{H}_{\hat{x}}^{\text {vel }}=\left(-\hat{\mathcal{T}}_{i}^{\text {vel }}\left[\boldsymbol{\omega}_{\text {moon }}^{i} \times\right], \hat{\mathcal{T}}_{i}^{\text {vel }},\left[\hat{\boldsymbol{v}}_{\text {rel }}^{\text {vel }} \times\right] \hat{\mathcal{T}}_{\ell}^{\text {vel }}, O_{3 \times 15},\right. \\
\left.O_{3 \times 23}, I_{3 \times 3}, \operatorname{Diag}\left(\hat{\boldsymbol{v}}_{\text {rel }}^{\text {vel }}\right),\left[\hat{\boldsymbol{v}}_{\text {rel }}^{\text {vel }} \times\right], O_{3 \times 15}, O_{3 \times 7}\right)
\end{array}
$$

The flight computer's value of the velocimeter measurement covariance is given by

$$
\hat{R}_{\nu}^{v e l}=\operatorname{Diag}\left(\left[\hat{\sigma}_{\text {vel }_{x}}^{2}, \hat{\sigma}_{\text {vely }_{y}}^{2}, \hat{\sigma}_{\text {vel }_{z}}^{2}\right]\right)
$$

The measurement sensitivity matrix for the TRN position sensor is given by

$$
\begin{gathered}
\hat{H}_{\hat{x}}^{t r n}=\left(\hat{\mathcal{T}}_{i}^{s}, O_{3 \times 3}, \hat{h} \hat{\mathcal{T}}_{\ell}^{s}\left[\hat{i}_{x}^{\ell} \times\right], O_{3 \times 12}, \hat{\mathcal{T}}_{i}^{s}\left\{-I_{3 \times 3}+\left[\hat{\boldsymbol{r}}_{\Delta r}^{s} \times\right]\left[\hat{\boldsymbol{r}}_{r}^{i} \times\right] /\left|\hat{\boldsymbol{r}}_{r}^{i}\right|^{2}\right\},\right. \\
\left.O_{3 \times 23}, I_{3 \times 3}, \operatorname{Diag}\left(\hat{\boldsymbol{r}}_{\Delta r}^{s}\right),-\hat{h} \hat{\mathcal{T}}_{\ell}^{s}\left[\hat{i}_{x}^{\ell} \times\right], O_{3 \times 6}, I_{3 \times 3}, O_{3 \times 4}\right)
\end{gathered}
$$

The flight computer's value of the TRN position sensor measurement covariance is given by

$$
\hat{R}_{\nu}^{t r n}=\operatorname{Diag}\left(\left[\hat{\sigma}_{t r n_{x}}^{2}, \hat{\sigma}_{t r n_{y}}^{2}, \hat{\sigma}_{t r n_{z}}^{2}\right]\right)
$$


The measurement sensitivity matrix for the range measurements to the first LRS is given by

$$
\hat{H}_{\hat{x}}^{\rho, l r s}=\left(\left(\hat{\boldsymbol{i}}_{r e l}^{i}\right)^{T}, O_{1 \times 6},-\left(\hat{\boldsymbol{i}}_{r e l}^{i}\right)^{T}, O_{1 \times 12}, O_{1 \times 41}, 1, O_{1 \times 5}, O_{1 \times 7}\right)
$$

where

$$
\hat{\boldsymbol{i}}_{r e l}^{i}=\left(\hat{\boldsymbol{r}}^{i}-\hat{\boldsymbol{r}}_{l r s}^{i}\right)^{T} /\left|\hat{\boldsymbol{r}}^{i}-\hat{\boldsymbol{r}}_{l r s}^{i}\right|
$$

The measurement sensitivity matrix for the range measurements to the second LRS and the LCT differ only in the indices. The flight computer's value of the range measurement covariance is given by

$$
\hat{R}_{\nu}^{\text {range }}=\operatorname{Diag}\left(\left[\hat{\sigma}_{\text {range }, \text { lrs } 1}^{2}, \hat{\sigma}_{\text {range }, \text { lrs } 2}^{2}, \hat{\sigma}_{\text {range }, \text { lct }}^{2}\right]\right)
$$

The measurement sensitivity matrix for the Doppler measurements to the first LRS is given by

$$
\hat{H}_{\hat{x}}^{\dot{\rho}, l r s}=\left(\frac{\partial \dot{\hat{\rho}}}{\partial \hat{\boldsymbol{r}}^{i}}, \hat{\boldsymbol{i}}_{r e l}^{i}, O_{1 \times 3}, \frac{\partial \dot{\hat{\rho}}}{\partial \hat{\boldsymbol{r}}_{l r s}^{i}},-\hat{\boldsymbol{i}}_{r e l}^{i}, O_{1 \times 9}, O_{1 \times 44}, 1, O_{1 \times 2}, O_{1 \times 7}\right)
$$

where

$$
\frac{\partial \dot{\hat{\rho}}}{\partial \hat{\boldsymbol{r}}^{i}}=\frac{\left(\hat{\boldsymbol{v}}^{i}-\hat{\boldsymbol{v}}_{l r s}^{i}\right)^{T}}{\left|\hat{\boldsymbol{r}}^{i}-\hat{\boldsymbol{r}}_{l r s}^{i}\right|}\left(I_{3 \times 3}-\hat{\boldsymbol{i}}_{r e l}^{i}\left(\hat{\boldsymbol{i}}_{r e l}^{i}\right)^{T}\right) \quad \text { and } \quad \frac{\partial \dot{\hat{\rho}}}{\partial \hat{\boldsymbol{r}}_{l r s}^{i}}=-\frac{\partial \dot{\hat{\rho}}}{\partial \hat{\boldsymbol{r}}^{i}}
$$

The measurement sensitivity matrix for the Doppler measurements to the second LRS differs only in the indices. The matrix for the LCT Doppler measurements, however, differs in that

$$
\frac{\partial \dot{\hat{\rho}}}{\partial \hat{\boldsymbol{r}}_{l c t}^{i}}=\frac{\left(\hat{\boldsymbol{v}}^{i}-\hat{\boldsymbol{r}}_{l c t}^{i} \times \boldsymbol{\omega}_{\text {moon }}^{i}\right)^{T}}{\left|\hat{\boldsymbol{r}}^{i}-\hat{\boldsymbol{r}}_{l c t}^{i}\right|}\left(I_{3 \times 3}-\hat{\boldsymbol{i}}_{\text {rel }}^{i}\left(\hat{\boldsymbol{i}}_{\text {rel }}^{i}\right)^{T}\right)-\left(\hat{\boldsymbol{i}}_{\text {rel }}^{i}\right)^{T}\left[\boldsymbol{\omega}_{\text {moon }}^{i} \times\right] .
$$

The flight computer's value of the Doppler measurement covariance is given by

$$
\hat{R}_{\nu}^{\text {doppler }}=\operatorname{Diag}\left(\left[\hat{\sigma}_{\text {doppler }, l r s 1}^{2}, \hat{\sigma}_{\text {doppler }, \text { lrs } 2}^{2}, \hat{\sigma}_{\text {doppler }, l c t}^{2}\right]\right)
$$




\section{Appendix B}

\section{Driving Factors for Radiometric Navigation Performance}

The two key driving factors behind how well radiometric navigation works in any situation are 1) the duration of the time for tracking and when it occurs, and 2) the relative geometry of the lander and the beacon and how much it changes over time. This is well demonstrated by the single orbiting and surface beacon cases.

\section{B.1 Single Orbiting Beacon Case Study}

The effects of these two key driving factors are most apparent when tracking data is used only during the final descent and not during the two orbits prior to the deorbit burn. The navigation performance at PDI for this situation is displayed in Fig. B.1. A definite structure can be observed in the figure, which becomes easier to understand when it is compared with the duration of time over which radiometric tracking is available (see Fig. B.2) and the maximum elevation reached by the LRS over the lander (see Fig. B.3).

\begin{tabular}{|c|c|c|c|c|c|c|c|c|c|c|c|}
\hline \multirow{2}{*}{\multicolumn{2}{|c|}{ PDI }} & \multicolumn{10}{|c|}{ LRS Mean Anomaly at Deorbit (deg) } \\
\hline & & 0 & 36 & 72 & 108 & 144 & 180 & 216 & 252 & 288 & 324 \\
\hline \multirow{4}{*}{$\begin{array}{l}\text { ర్రీ } \\
\frac{0}{0}\end{array}$} & -180 & 605 & 465 & 652 & 628 & 417 & 299 & 245 & 223 & 220 & 319 \\
\hline & -157.5 & 570 & 597 & 592 & 394 & 281 & 229 & 208 & 204 & 351 & 330 \\
\hline & -135 & 610 & 606 & 373 & 272 & 221 & 200 & 202 & 281 & 250 & 409 \\
\hline & -112.5 & 661 & 330 & 270 & 218 & 197 & 223 & 243 & 231 & 278 & 4090 \\
\hline \multirow{5}{*}{ 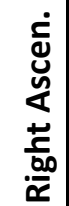 } & -90 & 715 & 261 & 220 & 197 & 278 & 227 & 222 & 266 & 367 & 4090 \\
\hline & -67.5 & 796 & 229 & 200 & 380 & 219 & 220 & 261 & 367 & 4090 & 4090 \\
\hline & -45 & 616 & 210 & 395 & 218 & 224 & 265 & 375 & 575 & 4090 & 4090 \\
\hline & -22.5 & 175 & 345 & 226 & 234 & 278 & 394 & 660 & 586 & 4090 & 4090 \\
\hline & 0 & 186 & 253 & 257 & 306 & 434 & 701 & 698 & 440 & 4090 & 4090 \\
\hline \multirow{7}{*}{ 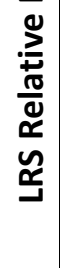 } & 22.5 & 189 & 305 & 361 & 514 & 749 & 692 & 501 & 388 & 4090 & 4090 \\
\hline & 45 & 236 & 446 & 672 & 797 & 639 & 502 & 439 & 444 & 4090 & 4090 \\
\hline & 67.5 & 456 & 727 & 720 & 564 & 478 & 449 & 456 & 453 & 4090 & 4090 \\
\hline & 90 & 523 & 543 & 468 & 440 & 447 & 475 & 514 & 590 & 4090 & 4090 \\
\hline & 112.5 & 624 & 395 & 392 & 442 & 494 & 562 & 662 & 678 & 4090 & 258 \\
\hline & 135 & 742 & 348 & 446 & 518 & 603 & 655 & 549 & 402 & 306 & 245 \\
\hline & 157.5 & 735 & 500 & 527 & 648 & 637 & 460 & 335 & 273 & 246 & 237 \\
\hline
\end{tabular}

Fig. B.1: Relative navigation results at PDI in meters $3-\boldsymbol{\sigma}$ using radiometric measurements to a single LRS only during the final descent. 


\begin{tabular}{|c|c|c|c|c|c|c|c|c|c|c|c|}
\hline \multirow{2}{*}{\multicolumn{2}{|c|}{$\begin{array}{c}\text { Duration of } \\
\text { Visibility ( } \mathrm{min} \text { ) }\end{array}$}} & \multicolumn{10}{|c|}{ LRS Mean Anomaly at Deorbit } \\
\hline & & 0 & 36 & 72 & 108 & 144 & 180 & 216 & 252 & 288 & 324 \\
\hline \multirow{4}{*}{ 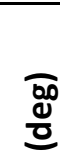 } & 0 & 11 & 9 & 16 & 21 & 27 & 32 & 37 & 42 & 41 & 31 \\
\hline & 22.5 & 10 & 10 & 20 & 27 & 33 & 37 & 41 & 45 & 42 & 29 \\
\hline & 45 & 10 & 13 & 26 & 34 & 38 & 41 & 44 & 46 & 41 & 22 \\
\hline & 67.5 & 10 & 22 & 35 & 39 & 42 & 43 & 44 & 45 & 38 & 0 \\
\hline \multirow{5}{*}{ 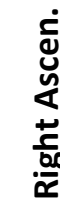 } & 90 & 10 & 36 & 42 & 43 & 43 & 43 & 43 & 42 & 27 & 0 \\
\hline & 112.5 & 11 & 46 & 45 & 45 & 43 & 42 & 40 & 36 & 0 & 0 \\
\hline & 135 & 19 & 50 & 47 & 44 & 42 & 39 & 34 & 25 & 0 & 0 \\
\hline & 157.5 & 51 & 52 & 47 & 42 & 38 & 33 & 26 & 16 & 0 & 0 \\
\hline & 180 & 52 & 52 & 44 & 38 & 32 & 26 & 20 & 12 & 0 & 0 \\
\hline \multirow{7}{*}{ 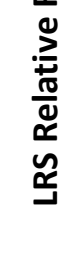 } & 202.5 & 52 & 50 & 38 & 31 & 25 & 21 & 16 & 11 & 0 & 0 \\
\hline & 225 & 48 & 41 & 28 & 23 & 20 & 18 & 15 & 11 & 0 & 0 \\
\hline & 247.5 & 39 & 19 & 19 & 19 & 18 & 16 & 15 & 12 & 0 & 0 \\
\hline & 270 & 28 & 11 & 15 & 16 & 17 & 17 & 16 & 14 & 0 & 0 \\
\hline & 292.5 & 21 & 9 & 14 & 16 & 17 & 18 & 19 & 19 & 0 & 19 \\
\hline & 315 & 16 & 9 & 13 & 16 & 18 & 21 & 24 & 28 & 29 & 29 \\
\hline & 337.5 & 13 & 8 & 14 & 18 & 22 & 26 & 31 & 37 & 37 & 32 \\
\hline
\end{tabular}

Fig. B.2: Duration of tracking during the final descent between the lander and a single LRS in minutes.

\begin{tabular}{|c|c|c|c|c|c|c|c|c|c|c|c|}
\hline \multirow{2}{*}{\multicolumn{2}{|c|}{$\begin{array}{c}\text { Maximum } \\
\text { Elevation (deg) }\end{array}$}} & \multicolumn{10}{|c|}{ LRS Mean Anomaly at Deorbit } \\
\hline & & 0 & 36 & 72 & 108 & 144 & 180 & 216 & 252 & 288 & 324 \\
\hline \multirow{4}{*}{ 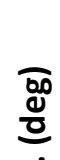 } & 0 & 8 & 18 & 34 & 41 & 43 & 44 & 46 & 52 & 64 & 71 \\
\hline & 22.5 & 12 & 18 & 34 & 41 & 46 & 53 & 61 & 73 & 86 & 35 \\
\hline & 45 & 19 & 18 & 34 & 44 & 55 & 66 & 79 & 85 & 57 & 9 \\
\hline & 67.5 & 26 & 18 & 38 & 55 & 69 & 83 & 82 & 62 & 31 & 0 \\
\hline \multirow{3}{*}{ 产 } & 90 & 29 & 24 & 51 & 70 & 86 & 80 & 63 & 42 & 8 & 0 \\
\hline & 112.5 & 26 & 40 & 69 & 88 & 77 & 63 & 47 & 27 & 0 & 0 \\
\hline & 135 & 19 & 64 & 89 & 74 & 61 & 50 & 37 & 22 & 0 & 0 \\
\hline \multirow{9}{*}{ 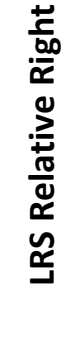 } & 157.5 & 16 & 89 & 68 & 57 & 49 & 42 & 36 & 22 & 0 & 0 \\
\hline & 180 & 43 & 61 & 49 & 45 & 43 & 42 & 35 & 22 & 0 & 0 \\
\hline & 202.5 & 80 & 36 & 36 & 41 & 43 & 42 & 35 & 22 & 0 & 0 \\
\hline & 225 & 61 & 19 & 34 & 41 & 43 & 42 & 35 & 22 & 0 & 0 \\
\hline & 247.5 & 35 & 18 & 34 & 41 & 43 & 42 & 35 & 22 & 0 & 0 \\
\hline & 270 & 20 & 18 & 34 & 41 & 43 & 42 & 35 & 22 & 0 & 0 \\
\hline & 292.5 & 12 & 18 & 34 & 41 & 43 & 42 & 35 & 22 & 0 & 4 \\
\hline & 315 & 7 & 18 & 34 & 41 & 43 & 42 & 36 & 25 & 16 & 31 \\
\hline & 337.5 & 6 & 18 & 34 & 41 & 43 & 42 & 38 & 35 & 38 & 67 \\
\hline
\end{tabular}

Fig. B.3: Maximum elevation reached by the LRS relative to the lander during the final descent in degrees. 
The most beneficial comparison is between the navigation accuracy and the amount of time for observation allowed by each case (Fig. B.1 and Fig. B.2) as it shows that the cases with 40 or more minutes of observation time during the final descent give the best navigation accuracy. Also, it explains the high plateau in the two right-most columns where no radiometric measurements are available during the final descent. As mentioned in Section 5.2.1 (page 43), whether the tracking occurs at the beginning of the descent trajectory (farleft column) or at the end of the descent trajectory (all other columns) plays a significant role in the navigation performance.

There are some trends in the data that cannot be explained by the duration of tracking such as the outliers in the center of the best results (the yellow diagonal in the center of the blue swath). Comparing the navigation accuracy and the maximum elevation attained by the LRS in the lander's LVLH frame (Fig. B.1 and Fig. B.3) offers an explanation. An elevation around 90 degrees indicates that the LRS orbits nearly directly over the lander. It was observed that in these cases, the LRS followed a ground track very similar to the ground track of the lander, which prevented the LRS from measuring the cross-track dispersion of the lander. In these cases, the navigation error is dominated by the cross-track component. Overall, cases where the LRS observes the lander from a wide range of directions provide the best navigation accuracies.

Comparing Fig. B.1 with Fig. 5.9 (page 45) shows that when radiometric tracking before the deorbit burn is used, the navigation results are much less sensitive to the right ascension and the mean anomaly, though some of the same trends can be observed (including the trajectories with the best results and a somewhat improved plateau where there is no tracking available during the final descent). The reason this occurs is because the additional tracking - even though it occurs early in the trajectory - improves the conditions of both of the factors discussed in this section. This same effect causes the navigation errors when two orbiting beacons are used to be much lower and much less sensitive to change. 


\section{B.2 Single Surface Beacon Case Study}

The same effects that were observed in the previous section can be noticed when a single surface beacon case is used for navigation, but with some variation. In order to conveniently show these effects, the navigation results of the coarse grid at landing, which were shown in Fig. 5.13 (page 51), are provided in Fig. B.4. As was mentioned in Section 5.2.3 (page

49), the most dominant effect on the navigation results was the off-track component of the position of the surface beacon. This can be observed in a comparison between the navigation results (Fig. B.4) and the maximum elevation attained by the lander over the surface beacon (Fig. B.7). Beacons that are on the ground track of the lander have maximum elevation angles near 90 degrees and yield very poor navigation accuracies due to their inability to adequately observe the cross-track dispersion of the lander.

When the LCT is located sufficiently far from the ground track of the descent trajectory, the effect of the maximum elevation is much less important than the duration of tracking (Fig. B.5) and the amount of time that separates the last measurement to the surface beacon and the final landing time (Fig. B.6). A combination of these two factors causes the remainder of the variation seen in the navigation results of the coarse grid.

\begin{tabular}{|c|c|c|c|c|c|c|c|c|c|c|c|c|}
\hline \multirow{2}{*}{\multicolumn{2}{|c|}{ Landing }} & \multicolumn{11}{|c|}{ Off-Track Location of Beacon (km) } \\
\hline & & \multirow[t]{2}{*}{80} & \multirow[t]{2}{*}{72} & \multirow[t]{2}{*}{64} & \multirow[t]{2}{*}{56} & \multirow[t]{2}{*}{48} & \multirow[t]{2}{*}{40} & \multirow[t]{2}{*}{32} & \multirow[t]{2}{*}{24} & \multirow{2}{*}{$\frac{16}{8}$} & \multirow{2}{*}{$\frac{8}{6}$} & \multirow{2}{*}{$\begin{array}{c}\mathbf{0} \\
70\end{array}$} \\
\hline$\overline{\boldsymbol{\varepsilon}}$ & 0 & & & & & & & & & & & \\
\hline 兰 & 35 & & & & & & 12 & 10 & 9 & 8 & 9 & 567 \\
\hline $\bar{u}$ & 70 & & & & & 15 & 14 & 13 & 12 & 12 & 14 & 504 \\
\hline Фّ & 105 & & & & 20 & 18 & 17 & 17 & 17 & 17 & 20 & 437 \\
\hline 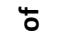 & 140 & & & 25 & 23 & 22 & 21 & 21 & 21 & 22 & 26 & 381 \\
\hline 으 & 175 & & & 28 & 27 & 26 & 25 & 25 & 26 & 27 & 32 & 337 \\
\hline 䓠 & 210 & & & 32 & 30 & 30 & 30 & 30 & 30 & 31 & 38 & 302 \\
\hline 9 & 245 & & 51 & 36 & 35 & 34 & 34 & 34 & 35 & 36 & 43 & 274 \\
\hline$\underset{0}{\infty}$ & 280 & & 42 & 40 & 40 & 39 & 39 & 39 & 39 & 41 & 48 & 252 \\
\hline$\underset{\mathbb{T}}{\mathscr{0}}$ & 315 & & 46 & 44 & 44 & 43 & 43 & 43 & 44 & 46 & 54 & 235 \\
\hline กำ & 350 & & 51 & 50 & 48 & 48 & 48 & 48 & 49 & 50 & 59 & 221 \\
\hline
\end{tabular}

Fig. B.4: Relative navigation results at landing in meters $3-\boldsymbol{\sigma}$ using radiometric measurements to a single LCT on the coarse grid. 


\begin{tabular}{|c|c|c|c|c|c|c|c|c|c|c|c|c|}
\hline \multirow{2}{*}{\multicolumn{2}{|c|}{$\begin{array}{c}\text { Duration of } \\
\text { Visibility (sec) }\end{array}$}} & \multicolumn{11}{|c|}{ Off-Track Location of Beacon (km) } \\
\hline & & 80 & 72 & 64 & 56 & 48 & 40 & 32 & 24 & 16 & 8 & $\mathbf{0}$ \\
\hline \multirow[t]{11}{*}{$\bar{\xi}$} & 0 & 0 & 0 & 0 & 0 & 0 & 0 & 0 & 0 & 58 & 100 & 221 \\
\hline & 35 & 0 & 0 & 0 & 0 & 0 & 39 & 74 & 95 & 108 & 116 & 119 \\
\hline & 70 & 0 & 0 & 0 & 0 & 53 & 76 & 91 & 102 & 110 & 113 & 115 \\
\hline & 105 & 0 & 0 & 0 & 46 & 69 & 83 & 94 & 101 & 107 & 110 & 111 \\
\hline & 140 & 0 & 0 & 29 & 56 & 73 & 85 & 93 & 99 & 103 & 105 & 107 \\
\hline & 175 & 0 & 0 & 39 & 59 & 72 & 83 & 90 & 95 & 99 & 101 & 102 \\
\hline & 210 & 0 & 0 & 42 & 60 & 71 & 80 & 87 & 91 & 94 & 96 & 97 \\
\hline & 245 & 0 & 8 & 43 & 58 & 68 & 72 & 76 & 87 & 89 & 90 & 90 \\
\hline & 280 & 0 & 13 & 41 & 48 & 64 & 68 & 71 & 83 & 85 & 86 & 86 \\
\hline & 315 & 0 & 10 & 40 & 47 & 62 & 65 & 78 & 80 & 82 & 83 & 83 \\
\hline & 350 & 0 & 10 & 30 & 50 & 60 & 64 & 77 & 79 & 80 & 81 & 81 \\
\hline
\end{tabular}

Fig. B.5: Duration of tracking between the lander and a single LCT on the coarse grid in seconds during the final descent.

\begin{tabular}{|c|c|c|c|c|c|c|c|c|c|c|c|c|}
\hline \multirow{2}{*}{\multicolumn{2}{|c|}{$\begin{array}{c}\text { Tgo After Loss of } \\
\text { Signal (sec) }\end{array}$}} & \multicolumn{11}{|c|}{ Off-Track Location of Beacon (km) } \\
\hline & & \multirow[t]{2}{*}{80} & \multirow[t]{2}{*}{72} & \multirow[t]{2}{*}{64} & \multirow[t]{2}{*}{56} & \multirow[t]{2}{*}{48} & \multirow[t]{2}{*}{40} & \multirow[t]{2}{*}{32} & \multirow[t]{2}{*}{24} & \multirow{2}{*}{$\frac{16}{152}$} & \multirow{2}{*}{$\frac{8}{119}$} & \multirow{2}{*}{0} \\
\hline \multirow{3}{*}{ 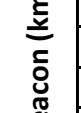 } & 0 & & & & & & & & & & & \\
\hline & 35 & & & & & & 217 & 196 & 182 & 173 & 167 & 165 \\
\hline & 70 & & & & & 246 & 232 & 223 & 216 & 211 & 209 & 208 \\
\hline \multirow{2}{*}{ 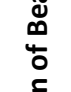 } & 105 & & & & 281 & 268 & 260 & 253 & 249 & 245 & 243 & 243 \\
\hline & 140 & & & 318 & 303 & 294 & 287 & 282 & 279 & 276 & 275 & 274 \\
\hline \multirow{3}{*}{ } & 175 & & & 338 & 327 & 320 & 314 & 310 & 307 & 305 & 304 & 303 \\
\hline & 210 & & & 361 & 351 & 345 & 340 & 336 & 334 & 332 & 331 & 330 \\
\hline & 245 & & 401 & 383 & 375 & 369 & 365 & 361 & 359 & 357 & 356 & 356 \\
\hline \multirow{3}{*}{ 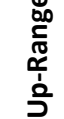 } & 280 & & 420 & 405 & 398 & 392 & 388 & 385 & 383 & 381 & 380 & 380 \\
\hline & 315 & & 446 & 426 & 419 & 414 & 411 & 408 & 406 & 404 & 403 & 403 \\
\hline & 350 & & 466 & 456 & 446 & 436 & 432 & 429 & 427 & 426 & 425 & 425 \\
\hline
\end{tabular}

Fig. B.6: Duration of time between the last radiometric measurement to and LCT on the coarse grid and landing in seconds.

\begin{tabular}{|c|c|c|c|c|c|c|c|c|c|c|c|c|}
\hline \multirow{2}{*}{\multicolumn{2}{|c|}{$\begin{array}{c}\text { Maximum } \\
\text { Elevation (deg) }\end{array}$}} & \multicolumn{11}{|c|}{ Off-Track Location of Beacon (km) } \\
\hline & & 80 & 72 & 64 & 56 & 48 & 40 & 32 & 24 & 16 & 8 & $\mathbf{0}$ \\
\hline \multirow{2}{*}{$\underline{\underline{g}}$} & 0 & 4 & 4 & 5 & 5 & 6 & 7 & 8 & 9 & 11 & 16 & 90 \\
\hline & 35 & 5 & 6 & 7 & 8 & 9 & 11 & 13 & 17 & 25 & 42 & 89 \\
\hline \multirow{2}{*}{ 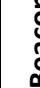 } & 70 & 6 & 7 & 8 & 10 & 12 & 14 & 17 & 23 & 32 & 52 & 88 \\
\hline & 105 & 7 & 8 & 10 & 11 & 13 & 16 & 20 & 26 & 36 & 56 & 88 \\
\hline 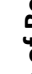 & 140 & 8 & 9 & 10 & 12 & 14 & 17 & 22 & 28 & 39 & 59 & 90 \\
\hline \multirow{2}{*}{ 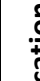 } & 175 & 8 & 10 & 11 & 13 & 15 & 18 & 23 & 29 & 40 & 60 & 89 \\
\hline & 210 & 9 & 10 & 11 & 13 & 16 & 19 & 23 & 30 & 41 & 61 & 88 \\
\hline 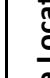 & 245 & 9 & 10 & 12 & 13 & 16 & 19 & 24 & 31 & 42 & 61 & 89 \\
\hline \multirow{2}{*}{ 每 } & 280 & 9 & 10 & 12 & 14 & 16 & 19 & 24 & 31 & 42 & 62 & 89 \\
\hline & 315 & 9 & 10 & 12 & 14 & 16 & 19 & 24 & 31 & 42 & 61 & 83 \\
\hline 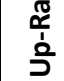 & 350 & 9 & 10 & 12 & 14 & 16 & 19 & 24 & 31 & 42 & 60 & 78 \\
\hline
\end{tabular}

Fig. B.7: Maximum elevation reached by the lander relative to the LCT during the final descent in degrees. 